Supporting Information for:

\title{
Vicinal, Double C-H Functionalization of Alcohols via an Imidate Radical-Polar Crossover Cascade
}

\author{
Allen F. Prusinowski, Raymond K. Twumasi, \\ Ethan A. Wappes, David A. Nagib*
}

Department of Chemistry and Biochemistry, The Ohio State University, 151 W. Woodruff Ave., Columbus, OH, 43210, United States

Corresponding Author

*E-mail: nagib.1@osu.edu 


\section{Table of Contents}

Section

Page

$\begin{array}{ll}\text { I. General Information S3 } & \text { S }\end{array}$

$\begin{array}{ll}\text { II. General Procedures } & \text { S4 }\end{array}$

$\begin{array}{lr}\text { III. Substrate Synthesis } & \text { S7 }\end{array}$

$\begin{array}{lr}\text { IV. Amino-halogenation Optimization } & \text { S28 }\end{array}$

$\begin{array}{ll}\text { V. Functional Group Tolerance and Additive Screen } & \text { S30 }\end{array}$

$\begin{array}{ll}\text { VI. Amino-iodination Characterization } & \text { S31 }\end{array}$

VII. Post-synthetic Functionalization Characterization $\quad$ S51

$\begin{array}{lr}\text { VIII. One-pot Procedure } & \text { S60 }\end{array}$

$\begin{array}{ll}\text { IX. C-H lodination } & \text { S61 }\end{array}$

$\begin{array}{lr}X . \text { Rate Experiments } & \text { S62 }\end{array}$

$\begin{array}{ll}\text { XI. Mechanistic Experiments and Reaction Profile } & \text { S70 }\end{array}$

$\begin{array}{ll}\text { XII. UV/Vis and NMR Experiments } & \text { S78 }\end{array}$

$\begin{array}{ll}\text { XIII. Electrochemical Data } & \mathrm{S} 80\end{array}$

XIV. X-ray Crystallographic Data $\quad$ S82

XV. One-Step Strategy $\quad$ S90

$\begin{array}{ll}X V . & \text { NMR Spectra }\end{array}$ 


\section{General Information}

All chemicals and reagents were purchased from Sigma-Aldrich, Alfa Aesar, Acros, TCl, Oakwood Chemical, or ChemImplex. Sodium iodide and reagents were dried under high vacuum before use. Solvents were purified in the following manner. Acetonitrile and amine bases were distilled over calcium hydride. $\mathrm{CH}_{2} \mathrm{Cl}_{2}, \mathrm{THF}, \mathrm{Et}_{2} \mathrm{O}$ and DMF were degassed with $\mathrm{N}_{2}$ and dried by passing through columns containing alumina, copper, or molecular sieves. Flash column chromatography, or preparative thin-layer chromatography, was performed with Silicycle F60 (230-400 mesh) silica gel. Thin layer chromatography (TLC) analyses were performed using EMD 60 F254 TLC plates and visualized by fluorescence quenching or $\mathrm{KMnO}_{4}$ stain. All yields are averages of at least two experimental runs. Cyclic voltammetry was performed with a BioLogic VSP potentiostat in a three-electrode electrochemical cell consisting of a glassy carbon disk working electrode $(0.07 \mathrm{~cm} 2, \mathrm{BASi}), \mathrm{a} \mathrm{Ag} / \mathrm{Ag}+$ quasi-reference electrode (BASi) containing $0.01 \mathrm{M} \mathrm{AgBF}$ (Sigma) in acetonitrile, and a platinum wire counter electrode $(23 \mathrm{~cm}, \mathrm{ALS})$. Charge/discharge measurements were carried out with a BioLogic VSP potentiostat in a custom glass $\mathrm{H}$-cell. Samples were prepared with $0.02 \mathrm{mmol}$ of analyte in $2.5 \mathrm{~mL}$ of $0.5 \mathrm{M}$ tetra-methylammonium tetrafluoroborate in dry, degassed acetonitrile. Data was analyzed using Excel. The onset potential ( $\left.E_{o n}\right)$ was measured by calculating the $x$-intercept potential between the linearly extrapolated fastest rising portion of the current wave (or peak) and the baseline (background) current.

Nuclear magnetic resonance (NMR) spectra $\left({ }^{1} \mathrm{H},{ }^{13} \mathrm{C}\right)$ were recorded using either a Bruker AVIII 400 or AVIII $600 \mathrm{MHz}$ NMR spectrometer. ${ }^{1} \mathrm{H}$ and ${ }^{13} \mathrm{C}$ NMR chemical shifts are reported in parts per million and referenced to residual $\mathrm{CHCl}_{3}$ signals in $\mathrm{CDCl}_{3}(1 \mathrm{H}: \delta$ 7.26; ${ }^{13} \mathrm{C}: \delta 77$.), or $\mathrm{CFCl}_{3}\left({ }^{19} \mathrm{~F}: \delta 0.0\right) .{ }^{1} \mathrm{H}$ NMR data are reported as follows: chemical shifts $(\delta \mathrm{ppm})$, multiplicity $(\mathrm{s}=$ singlet, $\mathrm{d}=$ doublet, $\mathrm{t}=$ triplet, $\mathrm{q}=$ quartet, quint $=$ quintet, $\mathrm{m}=$ multiplet, $\mathrm{b}=$ broad, ap = apparent), coupling constant $(\mathrm{Hz})$, relative integral. Data for ${ }^{13} \mathrm{C}$ is reported in terms of chemical shift and multiplicity where appropriate. Highresolution Mass Spectrometry (HRMS) data were obtained using Bruker MicrOTOF (ESI). Infrared (IR) spectra were recorded using a Thermo Fisher Nicolet iS10 FT-IR and are reported in terms of frequency of absorption $\left(\mathrm{cm}^{-1}\right)$. Melting points were determined using an Electrotherman IA9000.

Photochemical reactions were performed by placing two $26 \mathrm{~W}$ compact fluorescent light bulbs ( 1550 lumens, $5000 \mathrm{~K}$ ) approximately $2 \mathrm{~cm}$ away from the reaction vessel, with 1 fan to the side and another behind the reaction vessel. See a photograph of the set-up below. 


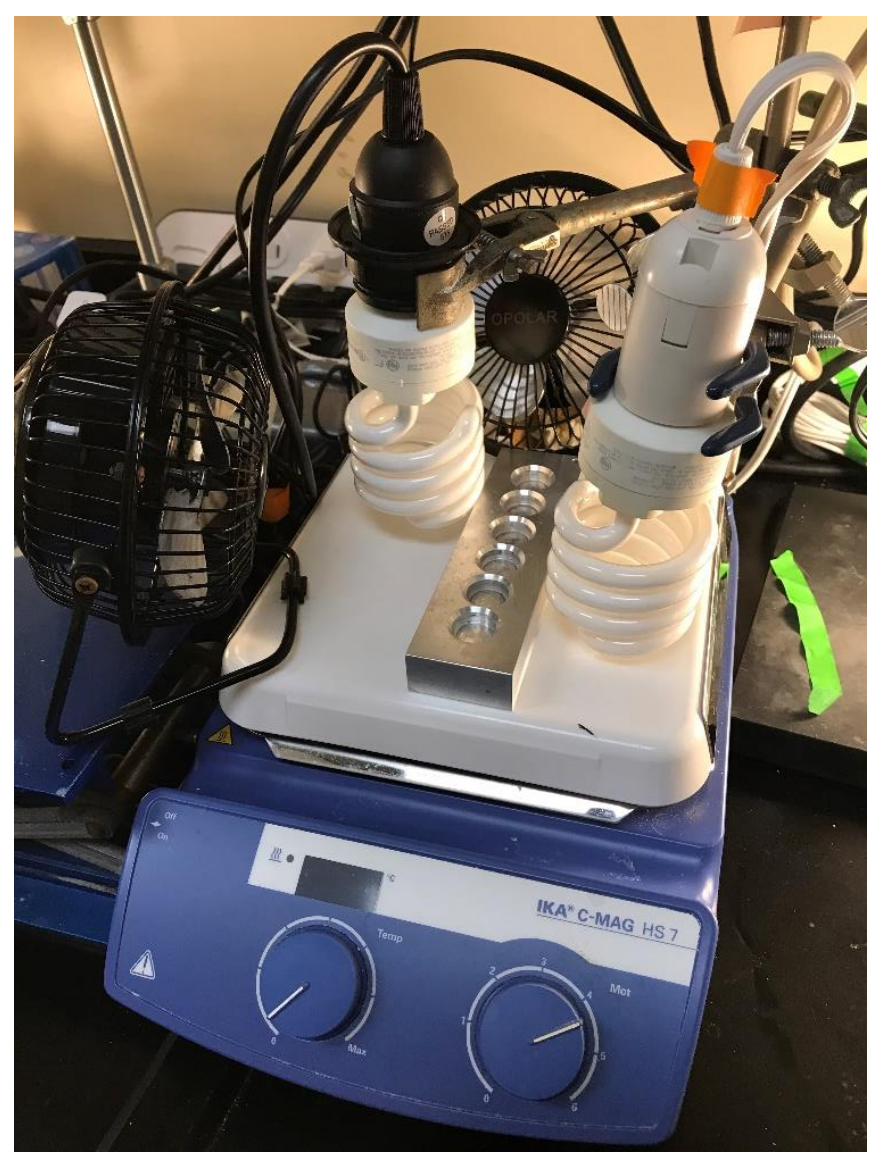

\section{General Procedures}

\section{General Procedure 1 (GP1) - Trichloroacetimidate Formation}

To a $100 \mathrm{~mL}$ round-bottom flask containing a stir bar, alcohol (1 equiv.), and $\mathrm{CH}_{2} \mathrm{Cl}_{2}$ (1 $\mathrm{M})$, was added trichloroacetonitrile (1.5 equiv.) and DBU (0.1 equiv.). The solution was stirred overnight and monitored by TLC. Upon completion, the solution was concentrated in vacuo before purification by flash chromatography (silica gel, specific eluent conditions noted with each substrate).

\section{Notes:}

- Silica gel is loaded with hexanes containing $1 \% \mathrm{Et}_{3} \mathrm{~N}$ to avoid imidate hydrolysis, which also results in quicker compound elution.

\section{General Procedure 2 (GP2) - Benzimidate Formation}

(a) To a 4-dram vial equipped with a PTFE septum cap and magnetic stir bar was added alcohol ( 1 equiv), nitrile (1.1 equiv), PhMe [0.5 M], and trifluoromethanesulfonic acid (1.2 equiv). The solution was heated to $110^{\circ} \mathrm{C}$ and stirred. After $24 \mathrm{~h}$ the solution was cooled to room temperature, and then stored at $-15{ }^{\circ} \mathrm{C}$ until crystallization of the 
trifluoromethanesulfonate salt was observed. The salt was isolated via filtration and washed with cold hexanes and $\mathrm{Et}_{2} \mathrm{O}$. Residual solvent was removed under vacuum.

(b) The salt was suspended in $\mathrm{Et}_{2} \mathrm{O}$, and $\mathrm{NaHCO}_{3}$ (sat. aq) was added dropwise until the dissolution of the salt observed. The aqueous layer was extracted $3 x$ with $\mathrm{CH}_{2} \mathrm{Cl}_{2}(5 \mathrm{~mL})$, and the combined organic layer was dried over anhydrous $\mathrm{MgSO}_{4}$ and concentrated in vacuo. The residue was purified via column chromatography (silica treated with $1 \%$ Et3 $\mathrm{N} /$ hexanes to avoid hydrolysis).

Notes:

- The free-based imidate is more prone to hydrolysis than the benzimidate salt, when stored for extended periods of time. For this reason, we suggest storing the benzimidate salt in a freezer and free-basing prior to use.

\section{General Procedure 3 (GP3) - Amino-lodination}

To a 2-dram vial equipped with a PTFE septum cap and magnetic stir bar, was added imidate (1 equiv.) and $\mathrm{I}_{2}$ (3 equiv.). Dry ${ }^{\mathrm{B}} \mathrm{BuOH}(0.3 \mathrm{M})$ was degassed using a freezepump-thaw technique (3x) or via sparging with $\mathrm{N}_{2}(15 \mathrm{~min})$, then added to the vial. This solution was stirred for 3 minutes, and iodobenzene diacetate ( 3 equiv.) was added as a solid. The headspace of this vial was evacuated and backfilled with $\mathrm{N}_{2}(3 \mathrm{x})$. The reaction was irradiated with two $26 \mathrm{~W}$ (1550 lumens, $5000 \mathrm{~K}$ ) compact fluorescent light bulbs and cooled by two fans for $1 \mathrm{~h}$. Upon completion, the solution was quenched via a reductive workup with $20 \% \mathrm{Na}_{2} \mathrm{~S}_{2} \mathrm{O}_{3}$. The organic layer was extracted with EtOAc (3x). The combined organic layer was concentrated in vacuo before purification by flash chromatography (specific eluent conditions noted with each substrate) to afford aminoiodinated product.

Notes:

- Solvent was degassed using a freeze-pump-thaw technique (3x)

- Pre-stir with imidate, $\mathrm{I}_{2}$, and ${ }^{\mathrm{t}} \mathrm{BuOH}$ is needed to fully ensure that $\mathrm{I}_{2}$ dissolves before adding iodobenzene diacetate to prevent degradation of diastereomeric ratio.

- lodobenzene diacetate must be sufficiently dry to ensure optimum yields. Typically, the material was left under high vacuum for at least $24 \mathrm{~h}$ before use.

- The reaction is heavily dependent on light setup. Ensuring optimum light bulb distance as well as obtaining the required light bulbs promote higher yields and diastereomeric ratios. 


\section{General Procedure 4 (GP4) - Amino-iodination for rate studies}

To a 2-dram vial equipped with a PTFE septa cap and magnetic stir bar was added imidate 1 (51.7 mg, $0.2 \mathrm{mmol}), \mathrm{I}_{2}(152.3 \mathrm{mg}, 0.6 \mathrm{mmol}),{ }^{\mathrm{t}} \mathrm{BuOH}(0.7 \mathrm{~mL})$, and dichloroethane as an internal standard $(15.5 \mu \mathrm{L}, 0.2 \mathrm{mmol})$. The solution was stirred at room temperature for $3 \mathrm{~min}$ to ensure $\mathrm{I}_{2}$ had been solubilized. lodobenzene diacetate was added as a solid, and the vial was immediately irradiated with $2 \times 26 \mathrm{~W}$ compact fluorescent light bulbs $(\sim 2 \mathrm{~cm})$ and stirred at $900 \mathrm{rpm}$. After the allotted time, an aliquot $(\sim 50 \mu \mathrm{L})$ was removed via syringe and immediately quenched in a vial containing $0.5 \mathrm{~mL}$ $\mathrm{CDCl}_{3}$ and $1.0 \mathrm{~mL} \mathrm{Na} \mathrm{S}_{2} \mathrm{O}_{3}(20 \%$, aq). After all time points had been collected, the organic layer was removed via syringe and dried over anhydrous $\mathrm{MgSO}_{4}$. The solution was directly analyzed by ${ }^{1} \mathrm{H}-\mathrm{NMR}, 600 \mathrm{MHz}, \mathrm{d}_{1}=3$ seconds.

\section{General Procedure 5 (GP5) - One-step amino-iodination}

To a 2-dram vial equipped with a PTFE septa cap and magnetic stir bar was added alcohol $(0.2 \mathrm{mmol}), \mathrm{I}_{2}(0.6 \mathrm{mmol})$, trichloroacetonitrile $(0.4 \mathrm{mmol})$, DBU $(0.02 \mathrm{mmol})$, and $\mathrm{PhCF}_{3}(0.7 \mathrm{~mL})$. The solution was stirred at room temperature for 3 min to ensure $\mathrm{I}_{2}$ had been solubilized. lodobenzene diacetate $(0.2 \mathrm{mmol})$ was added as a solid, and the vial was immediately irradiated with $2 \times 26 \mathrm{~W}$ compact fluorescent light bulbs $(\sim 2 \mathrm{~cm})$ and stirred at $900 \mathrm{rpm}$. After $24 \mathrm{~h}, \mathrm{PhCF}_{3}$ was removed and replaced with $1.0 \mathrm{~mL} \mathrm{MeCN}$ and $0.5 \mathrm{~mL} \mathrm{H} \mathrm{H}_{2} \mathrm{O}$. TsOH (1 equiv.) was added, and the reaction was stirred until no oxazoline was observed by thin layer chromatography. Upon completion, the solution was quenched via a reductive workup with $20 \% \mathrm{Na}_{2} \mathrm{~S}_{2} \mathrm{O}_{3}$ and $\mathrm{NaHCO}_{3}$ (sat. aq.). The organic layer was extracted with EtOAc (3x). The combined organic layer was concentrated in vacuo before purification by flash chromatography (specific eluent conditions noted with each substrate) to afford amino-iodinated product. 


\section{Substrate Synthesis}

\section{Alcohols}<smiles>O=C(OCC1CCC(CO)CC1)c1ccc(C(F)(F)F)cc1</smiles>

\section{(4-(hydroxymethyl)cyclohexyl)methyl 4-(trifluoromethyl)benzoate (S12A)}

Cyclohexane-1,4-diyldimethanol $(7.21 \mathrm{~g}, 50.0 \mathrm{mmol})$ was dissolved in THF $(75 \mathrm{~mL})$ and cooled to $0^{\circ} \mathrm{C}$. $\mathrm{NaH}(60 \%$ dispersion in oil) $(2.0 \mathrm{~g}, 50.0 \mathrm{mmol})$ was added portionwise, and the suspension was stirred for $1 \mathrm{~h}$. At $0^{\circ} \mathrm{C}, 4$-(trifluoromethyl)benzoyl chloride (6.95 $\mathrm{g}$, $4.9 \mathrm{~mL}, 33.3 \mathrm{mmol}$ ) was added dropwise by syringe and allowed to stir at room temperature for $6 \mathrm{~h}$. The reaction was quenched with $\mathrm{H}_{2} \mathrm{O}(20 \mathrm{~mL})$ and $\mathrm{Et}_{2} \mathrm{O}(20 \mathrm{~mL})$. The solution was extracted $5 x$ with $\mathrm{Et}_{2} \mathrm{O}(20 \mathrm{~mL})$. The combined organic layer was dried over $\mathrm{MgSO}_{4}$ (anhydrous) and concentrated in vacuo. The residue was adsorbed onto celite, loaded onto silica gel, and purified by flash column chromatography (isocratic: 20 : 80 EtOAc : hexanes) to yield the target alcohol S12A $(7.34 \mathrm{~g}, 70 \%)$ as a colorless oil.

Rf: 0.21 (20\% EtOAc/hexanes).

${ }^{1} \mathrm{H}$ NMR (600 MHz, CDCl 3 ): $\delta=8.15(\mathrm{~d}, \mathrm{~J}=7.9 \mathrm{~Hz}, 2 \mathrm{H}), 7.70(\mathrm{~d}, \mathrm{~J}=8.2 \mathrm{~Hz}, 2 \mathrm{H}), 4.29$ $(\mathrm{d}, \mathrm{J}=7.2 \mathrm{~Hz}, 1 \mathrm{H}), 4.19(\mathrm{~d}, \mathrm{~J}=6.4 \mathrm{~Hz}, 1 \mathrm{H}), 3.57(\mathrm{~d}, \mathrm{~J}=7.0 \mathrm{~Hz}, 1 \mathrm{H}), 3.48(\mathrm{~d}, 6.2 \mathrm{~Hz}, 1 \mathrm{H})$, 2.07-1.86 (m, 2H), 1.81-1.70 (m, 1H), 1.65-1.58 (m, 2H), 1.55-1.45 (m, 3H), $1.37(\mathrm{bs}, 1 \mathrm{H})$, 1.16-1.09 (m, 1H), 1.06-0.99 (m, 1H).

${ }^{13} \mathrm{C}$ NMR (150 MHz, CDCl 3 ): $\delta=165.57,165.54,134.52$ (q, J = $32.8 \mathrm{~Hz}$ ), 133.81, 133.78, 130.08, 125.54 (q, 3.5 Hz), 123.77 (q, 272.8 Hz), 70.54, 68.59, 68.31, 66.23, 40.52, 38.19, $37.51,34.73,29.20,28.84,25.68,25.24$.

HRMS (ESI-TOF) m/z: calc'd for $\mathrm{C}_{16} \mathrm{H}_{19} \mathrm{~F}_{3} \mathrm{O}_{3}[\mathrm{M}+\mathrm{Na}]^{+} 339.1184$, found 339.1171.

IR (film) cm-1: 3345, 3021, 2978, 2922, 2856, 1720, 1324, 1098, 1017, 861.

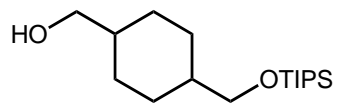

\section{(4-(((triisopropylsilyl)oxy)methyl)cyclohexyl)methanol (S13A)}

Cyclohexane-1,4-diyldimethanol $(7.21 \mathrm{~g}, 50.0 \mathrm{mmol})$ was dissolved in THF $(75 \mathrm{~mL})$ and cooled to $0^{\circ} \mathrm{C}$. $\mathrm{NaH}(60 \%$ dispersion in oil) $(2.0 \mathrm{~g}, 50.0 \mathrm{mmol})$ was added portionwise, and the suspension was stirred for $1 \mathrm{~h}$. At $0^{\circ} \mathrm{C}$, TIPSCl $(6.42 \mathrm{~g}, 7.1 \mathrm{~mL}, 33.3 \mathrm{mmol})$ was added dropwise by syringe and allowed to stir at room temperature for $6 \mathrm{~h}$. The reaction was quenched with $\mathrm{H}_{2} \mathrm{O}(20 \mathrm{~mL})$ and $\mathrm{Et}_{2} \mathrm{O}(20 \mathrm{~mL})$. The solution was extracted $5 x$ with 
$\mathrm{Et}_{2} \mathrm{O}(20 \mathrm{~mL})$. The combined organic layer was dried over $\mathrm{MgSO}_{4}$ (anhydrous) and concentrated in vacuo. The residue was adsorbed onto celite, loaded onto silica gel, and purified by flash column chromatography (isocratic: $20: 80$ EtOAc : hexanes) to yield the target alcohol S13A (5.6 g, 56\%) as a colorless oil.

Rf: 0.42 (20\% EtOAc/hexanes).

${ }^{1} \mathrm{H}$ NMR $\left(600 \mathrm{MHz}, \mathrm{CDCl}_{3}\right): \delta=3.56(\mathrm{~d}, \mathrm{~J}=5.1 \mathrm{~Hz}, 1 \mathrm{H}), 3.55(\mathrm{~d}, \mathrm{~J}=5.3 \mathrm{~Hz}, 1 \mathrm{H}), 3.49$ $(\mathrm{d}, \mathrm{J}=6.2,1 \mathrm{H}), 3.45(\mathrm{~d}, \mathrm{~J}=6.3 \mathrm{~Hz}, 1 \mathrm{H}), 1.83-1.81(\mathrm{~m}, 3 \mathrm{H}), 1.71-1.65(\mathrm{~m}, 1 \mathrm{H}), 1.55-1.49$ (m, 2H), 1.45-1.41 (m, 4H), $1.36(\mathrm{bs}, 1 \mathrm{H}), 1.05(\mathrm{~s}, 18 \mathrm{H})$.

${ }^{13} \mathrm{C}$ NMR (150 MHz, $\left.\mathrm{CDCl}_{3}\right): \delta=69.08,68.91,66.79,66.17,41.06,40.92,38.58,38.19$, 29.19, 29.17, 25.71, 25.71, 25.38, 18.19, 12.15.

HRMS (ESI-TOF) m/z: calc'd for $\mathrm{C}_{17} \mathrm{H}_{36} \mathrm{O}_{2} \mathrm{Si}[\mathrm{M}+\mathrm{H}]^{+}$301.2563, found 301.2537.

IR (film) cm$^{-1}:$ 3329, 2940, 2918, 2864, 1463,1116, 1059, 1032, 881. 
Imidates

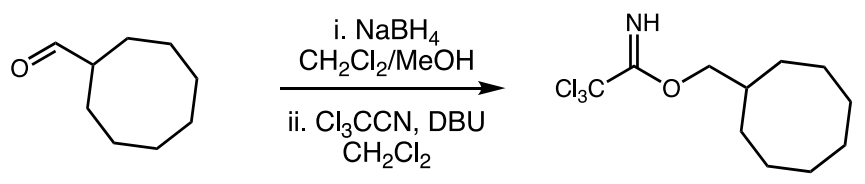

cyclooctylmethyl 2,2,2-trichloroacetimidate (S6)

Step 1: Aldehyde reduction

To a solution of cyclooctanecarbaldehyde $(1.08 \mathrm{~mL}, 7.13 \mathrm{mmol})$ in a $1: 1$ mixture of $\mathrm{CH}_{2} \mathrm{Cl}_{2}$ and $\mathrm{MeOH}(35 \mathrm{~mL})$ was slowly added $\mathrm{NaBH}_{4}$ portionwise $(1.06 \mathrm{~g}, 28.5 \mathrm{mmol})$ at $0^{\circ} \mathrm{C}$. The mixture was warmed to room temperature and stirred for $1 \mathrm{~h}$. Upon completion (monitored by $\mathrm{TLC})$, the reaction was quenched with $\mathrm{H}_{2} \mathrm{O}(35 \mathrm{~mL})$ under vigorous stirring, and extracted with $\mathrm{CH}_{2} \mathrm{Cl}_{2}$. The combined organic layer was dried over anhydrous $\mathrm{MgSO}_{4}$ and concentrated in vacuo. The crude mixture of cyclooctylmethanol was carried forward without further purification.

Step 2: Trichloroacetimidate formation

The crude mixture was subjected to GP1 without further purification. Upon completion (monitored by TLC) the reaction was concentrated and loaded directly onto silica gel and purified by flash column chromatography (isocratic: $10: 89: 1$, EtOAc : hexane : Et ${ }_{3} \mathrm{~N}$ ) to yield imidate $\mathbf{S 6}(2.01 \mathrm{~g}, 98 \%$ over two steps) as a colorless oil.

Rf: 0.41 (5\% EtOAc/hexanes)

${ }^{1} \mathrm{H}$ NMR $\left(400 \mathrm{MHz}, \mathrm{CDCl}_{3}\right): \delta=8.20(\mathrm{bs}, 1 \mathrm{H}), 4.06(\mathrm{~d}, \mathrm{~J}=6.9 \mathrm{~Hz}, 2 \mathrm{H}), 2.08-1.99(\mathrm{~m}$, $1 \mathrm{H}), 1.76-1.67(\mathrm{~m}, 4 \mathrm{H}), 1.59-1.56(\mathrm{~m}, 8 \mathrm{H}), 1.53-1.50(\mathrm{~m}, 2 \mathrm{H})$.

${ }^{13} \mathrm{C}$ NMR (100 MHz, $\left.\mathrm{CDCl}_{3}\right): \delta=163.32,91.88,75.08,36.81,29.25,27.06,26.48,25.48$.

HRMS (ESI-TOF) m/z: calc'd for $\mathrm{C}_{11} \mathrm{H}_{18} \mathrm{Cl}_{3} \mathrm{NO}[\mathrm{M}+\mathrm{Na}]^{+} 308.0346$, found 308.0348 .

IR (neat) cm$^{-1}:$ 3345, 2917, 2850, 1661, 1466, 1075, 793.

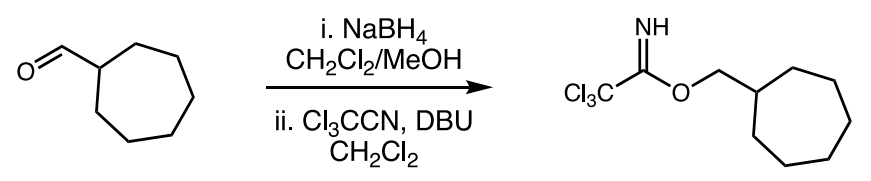

\section{cycloheptylmethyl 2,2,2-trichloroacetimidate (S7)}

Step 1: Carboxylic acid reduction

To a suspension of $\mathrm{LiAlH}_{4}(0.3985 \mathrm{~g}, 10.5 \mathrm{mmol})$ in THF $(77 \mathrm{~mL})$ under $\mathrm{N}_{2}$ was slowly added cycloheptanecarboxylic acid $(0.96 \mathrm{~mL}, 7 \mathrm{mmol})$ in THF $(23 \mathrm{~mL})$ dropwise at $0^{\circ} \mathrm{C}$. The mixture was warmed to room temperature and stirred overnight. Upon completion 
(monitored by $\mathrm{TLC}$ ), the reaction was diluted with $\mathrm{Et}_{2} \mathrm{O}$ and cooled to $0^{\circ} \mathrm{C}$. The reaction was quenched by adding $\mathrm{H}_{2} \mathrm{O}(0.40 \mathrm{~mL}), 10 \% \mathrm{NaOH}(0.40 \mathrm{~mL})$, and $\mathrm{H}_{2} \mathrm{O}(1.2 \mathrm{~mL})$ under vigorous stirring. The reaction was allowed to warm to room temperature for 15 minutes, and extracted with $\mathrm{Et}_{2} \mathrm{O}$. The combined organic layer was dried over anhydrous $\mathrm{MgSO}_{4}$ and concentrated in vacuo. The crude mixture of cycloheptylmethanol was carried forward without further purification.

Step 2: Trichloroacetimidate formation

The crude mixture was subjected to GP1 without further purification. Upon completion (monitored by TLC) the reaction was concentrated and loaded directly onto silica gel and purified by flash column chromatography (isocratic: $10: 89: 1$, EtOAc : hexane : $\mathrm{Et}_{3} \mathrm{~N}$ ) to yield imidate $\mathbf{S} 7$ (1.76 g, 92\% over two steps) as a yellow oil.

Rf: 0.43 (5\% EtOAc/hexanes)

${ }^{1} \mathrm{H}$ NMR (400 MHz, $\left.\mathrm{CDCl}_{3}\right): \delta=8.20(\mathrm{bs}, 1 \mathrm{H}), 4.08(\mathrm{~d}, \mathrm{~J}=6.9 \mathrm{~Hz}, 2 \mathrm{H}), 2.05-1.95(\mathrm{~m}$, $1 \mathrm{H}), 1.86-1.79(\mathrm{~m}, 2 \mathrm{H}), 1.76-1.42(\mathrm{~m}, 8 \mathrm{H}), 1.35-1.26(\mathrm{~m}, 2 \mathrm{H})$.

${ }^{13} \mathrm{C}$ NMR (100 MHz, $\left.\mathrm{CDCl}_{3}\right): \delta=163.36,91.91,74.78,38.54,31.03,28.71,26.45$.

HRMS (ESI-TOF) m/z: calc'd for $\mathrm{C}_{10} \mathrm{H}_{16} \mathrm{Cl}_{3} \mathrm{NO}[\mathrm{M}+\mathrm{Na}]^{+} 294.0190$, found 294.0168 .

IR (neat) $\mathbf{c m}^{-1}:$ 3345, 2921, 2853, 1661, 1461, 1308, 1076, 991.

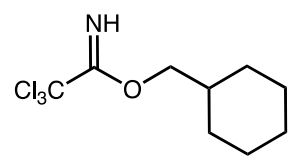

\section{cyclohexylmethyl 2,2,2-trichloroacetimidate (1)}

Cyclohexylmethanol $(2.15 \mathrm{~mL}, 17.5 \mathrm{mmol})$ was subjected to GP1. Upon completion (monitored by TLC) the reaction was concentrated and loaded directly onto silica gel and purified by flash column chromatography (isocratic: $10: 89: 1$, EtOAc : hexane : $\mathrm{Et}_{3} \mathrm{~N}$ ) to yield imidate 1 (4.52 g, 91\%) as a colorless oil.

Rf: 0.47 (5\% EtOAc/hexanes)

${ }^{1} \mathrm{H}$ NMR $\left(400 \mathrm{MHz}, \mathrm{CDCl}_{3}\right): \delta=8.20(\mathrm{bs}, 1 \mathrm{H}), 4.09(\mathrm{~d}, \mathrm{~J}=6.0 \mathrm{~Hz}, 2 \mathrm{H}), 1.86-1.66(\mathrm{~m}$, $6 \mathrm{H}), 1.34-1.00(\mathrm{~m}, 5 \mathrm{H})$.

${ }^{13} \mathrm{C}$ NMR (100 MHz, $\left.\mathrm{CDCl}_{3}\right): \delta=163.35,91.88,74.78,37.12,29.69,26.51,25.83$.

HRMS (ESI-TOF) m/z: calc'd for $\mathrm{C}_{9} \mathrm{H}_{14} \mathrm{Cl}_{3} \mathrm{NO}[\mathrm{M}+\mathrm{Na}]^{+} 280.0033$, found 280.0025 .

IR (neat) cm$^{-1}:$ 3346, 2924, 2852, 1661, 1465, 1075, 821, 793. 


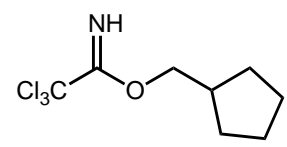

cyclopentylmethyl 2,2,2-trichloroacetimidate (S8)

Cyclopentanemethanol $(0.5 \mathrm{~g}, 0.54 \mathrm{~mL}, 5.0 \mathrm{mmol})$ was subjected to GP1. Upon completion (monitored by TLC) the reaction was concentrated and loaded directly onto silica gel and purified by flash column chromatography (isocratic: $5: 94: 1$, EtOAc : hexanes : Et $3 \mathrm{~N})$ to yield imidate $\mathbf{S 8}(1.2 \mathrm{~g}, 100 \%)$ as a light yellow oil.

Rf: 0.57 (10\% EtOAc / hexanes).

${ }^{1} \mathrm{H}$ NMR (400 MHz, $\left.\mathrm{CDCl}_{3}\right): \delta=8.20(\mathrm{bs}, 1 \mathrm{H}), 4.17(\mathrm{~d}, \mathrm{~J}=7.0 \mathrm{~Hz}, 2 \mathrm{H}), 2.38$ (sept, J = 7.5 $\mathrm{Hz}, 1 \mathrm{H}), 1.85-1.77(\mathrm{~m}, 2 \mathrm{H}), 1.69-1.54(\mathrm{~m}, 4 \mathrm{H}), 1.40-1.31(\mathrm{~m}, 2 \mathrm{H})$.

${ }^{13} \mathrm{C}$ NMR (100 MHz, $\left.\mathrm{CDCl}_{3}\right): \delta=163.3,91.9,73.5,38.4,29.4,25.5$.

HRMS (ESI-TOF) m/z: calc'd for $\mathrm{C}_{8} \mathrm{H}_{12} \mathrm{Cl}_{3} \mathrm{NO}[\mathrm{M}+\mathrm{Na}]^{+}$265.9882, found 265.9886.

IR (film) cm': 3345, 2952, 2868, 1662, 1076.

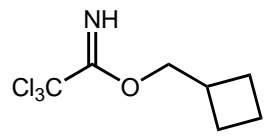

cyclobutylmethyl 2,2,2-trichloroacetimidate (S9)

Cyclobutylmethanol (0.66 mL, $7 \mathrm{mmol}$ ) was subjected to GP1. Upon completion (monitored by TLC) the reaction was concentrated and loaded directly onto silica gel and purified by flash column chromatography (isocratic: $10: 89: 1$, EtOAc : hexane : Et $3 \mathrm{~N}$ ) to yield imidate S9 (1.59 $\mathrm{g}, 99 \%)$ as a yellow oil.

Rf: 0.35 (5\% EtOAc/hexanes)

${ }^{1} \mathrm{H}$ NMR $\left(400 \mathrm{MHz}, \mathrm{CDCl}_{3}\right): \delta=8.22(\mathrm{bs}, 1 \mathrm{H}), 4.26(\mathrm{~d}, \mathrm{~J}=6.4 \mathrm{~Hz}, 2 \mathrm{H}), 2.84-2.73(\mathrm{~m}$, $1 \mathrm{H}), 2.15-2.08(\mathrm{~m}, 2 \mathrm{H}), 1.99-1.88(\mathrm{~m}, 4 \mathrm{H})$.

${ }^{13} \mathrm{C}$ NMR (100 MHz, $\left.\mathrm{CDCl}_{3}\right): \delta=163.37,91.86,73.12,34.02,24.69,18.61$.

HRMS (ESI-TOF) m/z: calc'd for $\mathrm{C}_{7} \mathrm{H}_{10} \mathrm{Cl}_{3} \mathrm{NO}[\mathrm{M}+\mathrm{Na}]^{+} 251.9720$, found 251.9720.

IR (neat) cm$^{-1}:$ 3346, 2941, 1662, 1460, 1291, 1073, 792. 
<smiles>N=C(OCC1CCOCC1)C(Cl)(Cl)Cl</smiles>

(tetrahydro-2H-pyran-4-yl)methyl 2,2,2-trichloroacetimidate (S10)

(Tetrahydro-2H-pyran-4-yl)methanol $(0.58 \mathrm{~g}, 5.0 \mathrm{mmol})$ was subjected to GP1. Upon completion (monitored by TLC) the reaction was concentrated and loaded directly onto silica gel and purified by flash column chromatography (isocratic: $5: 94: 1$, EtOAc : hexanes : Et $\left.{ }_{3} \mathrm{~N}\right)$ to yield imidate $\mathbf{S} 10(1.1 \mathrm{~g}, 85 \%)$ as a colorless oil that solidified over time to give a white solid.

Rf: 0.56 (40\% EtOAc / hexanes).

${ }^{1} \mathrm{H}$ NMR $\left(400 \mathrm{MHz}, \mathrm{CDCl}_{3}\right): \delta=8.26(\mathrm{bs}, 1 \mathrm{H}), 4.14(\mathrm{~d}, \mathrm{~J}=6.5 \mathrm{~Hz}, 2 \mathrm{H}), 4.02-3.98(\mathrm{~m}, 2 \mathrm{H})$, $3.41(\mathrm{td}, \mathrm{J}=17.7,2.1 \mathrm{~Hz}, 2 \mathrm{H}), 2.15-2.04(\mathrm{~m}, 1 \mathrm{H}), 1.73-1.63(\mathrm{~m}, 2 \mathrm{H}), 1.51-1.41(\mathrm{~m}, 2 \mathrm{H})$.

${ }^{13} \mathrm{C}$ NMR (100 MHz, $\left.\mathrm{CDCl}_{3}\right): \delta=163.10,91.65,73.64,67.61,34.52,29.51$.

HRMS (ESI-TOF) m/z: calc'd for $\mathrm{C}_{8} \mathrm{H}_{12} \mathrm{Cl}_{3} \mathrm{NO}_{2}[\mathrm{M}+\mathrm{H}]^{+} 260.0012$, found 260.0001 .

IR (film) cm$^{-1}:$ 3226, 1650, 1104.

MP: $137-139^{\circ} \mathrm{C}$.<smiles></smiles>

\section{(1-tosylpiperidin-4-yl)methyl 2,2,2-trichloroacetimidate (S11)}

1-tosylpiperidin-4-yl)methanol (1.35 g, $5 \mathrm{mmol}$ ) was subjected to GP1. Upon completion (monitored by TLC) the reaction was concentrated and loaded directly onto silica gel and purified by flash column chromatography (isocratic: $15: 84: 1$, EtOAc : hexanes : Et ${ }_{3} N$ ) to yield imidate $\mathbf{S} 11(0.83 \mathrm{~g}, 100 \%)$ as a light yellow solid.

Rf: 0.63 (40\% EtOAc / hexanes).

'1H NMR (400 MHz, $\left.\mathrm{CDCl}_{3}\right): \delta=8.25(\mathrm{bs}, 1 \mathrm{H}), 7.65(\mathrm{~d}, \mathrm{~J}=8.2 \mathrm{~Hz}, 2 \mathrm{H}), 7.32(\mathrm{~d}, \mathrm{~J}=8.5$ $\mathrm{Hz}, 2 \mathrm{H}), 4.11(\mathrm{~d}, \mathrm{~J}=6.2,2 \mathrm{H}), 3.84-3.81(\mathrm{~m}, 2 \mathrm{H}), 2.43(\mathrm{~s}, 3 \mathrm{H}), 2.29(\mathrm{td}, \mathrm{J}=18.0,2.6 \mathrm{~Hz}$, $2 \mathrm{H}), 1.85-1.72(\mathrm{~m}, 3 \mathrm{H}), 1.53-1.43(\mathrm{~m}, 2 \mathrm{H})$.

${ }^{13} \mathrm{C}$ NMR (150 MHz, $\left.\mathrm{CDCl}_{3}\right): \delta=162.93,143.64,133.34,129.76,127.86,91.50,72.90$, $46.04,34.77,28.18,21.66$. 
HRMS (ESI-TOF) m/z: calc'd for $\mathrm{C}_{15} \mathrm{H}_{19} \mathrm{Cl}_{3} \mathrm{~N}_{2} \mathrm{O}_{3} \mathrm{~S}$ [M+Na]+ 435.0080, found 435.0108 .

IR (film) cm$^{-1}:$ 3312, 3051, 1669, 1381, 1163, 1078.

MP: $113-114^{\circ} \mathrm{C}$.<smiles>N=C(OCC1CCC(COC(=O)c2ccc(C(F)(F)F)cc2)CC1)C(F)(Cl)Cl</smiles>

(4-((2,2,2-trichloro-1-iminoethoxy)methyl)cyclohexyl)methyl 4(trifluoromethyl)benzoate (S12)

(4-(hydroxymethyl)cyclohexyl)methyl 4-(trifluoromethyl)benzoate $(1.58 \mathrm{~g}, 5.0 \mathrm{mmol}$ ) was subjected to GP1. Upon completion (monitored by TLC) the reaction was concentrated and loaded directly onto silica gel and purified by flash column chromatography (isocratic: 5 : 94 : 1, EtOAc : hexanes : $\left.\mathrm{Et}_{3} \mathrm{~N}\right)$ to yield imidate $\mathbf{S} 12(2.13 \mathrm{~g}, 93 \%)$ as a colorless oil.

Rf: 0.29 (10\% EtOAc/hexanes).

${ }^{1} \mathrm{H}$ NMR (600 MHz, CDCl 3$): \delta=8.24(\mathrm{bs} \mathrm{J}=9.8 \mathrm{~Hz}, 1 \mathrm{H}), 8.15(\mathrm{~d}, \mathrm{~J}=8.2 \mathrm{~Hz}, 2 \mathrm{H}), 7.69$ $(\mathrm{d}, \mathrm{J}=8.3 \mathrm{~Hz}, 2 \mathrm{H}), 4.29(\mathrm{~d}, \mathrm{~J}=7.3 \mathrm{~Hz}, 1 \mathrm{H}), 4.23(\mathrm{~d}, \mathrm{~J}=7.1 \mathrm{~Hz}, 1 \mathrm{H}), 4.19(\mathrm{~d}, \mathrm{~J}=6.4 \mathrm{~Hz}$, $1 \mathrm{H}), 4.12(\mathrm{~d}, \mathrm{~J}=6.3 \mathrm{~Hz}, 1 \mathrm{H}), 2.10-2.04(\mathrm{~m}, 1 \mathrm{H}), 1.94-1.92(\mathrm{~m}, 2 \mathrm{H}), 1.84-1.79(\mathrm{~m}, 1 \mathrm{H})$, 1.68-1.64 (m, 2H), 1.58-1.53 (m, 2H), 1.19-1.12 (m, 2H).

${ }^{13} \mathrm{C}$ NMR (150 MHz, $\left.\mathrm{CDCl}_{3}\right): \delta=165.48,163.22,163.19,134.52$ (q, $\left.32.6 \mathrm{~Hz}\right), 133.76$, $130.07,125.52$ (q, 3.8 Hz), 123.77 (q, 272.6 Hz), 91.76, 91.74, 74.33, 72.22, 70.40, 68.24, $37.29,37.04,34.70,34.40,29.03,28.81,25.50,25.30$.

${ }^{19} \mathrm{~F}$ NMR (564 MHz, $\left.\mathrm{CDCl}_{3}\right): \delta=-63.11$.

HRMS (ESI-TOF) m/z: calc'd for $\mathrm{C}_{18} \mathrm{H}_{19} \mathrm{Cl}_{3} \mathrm{~F}_{3} \mathrm{NO}_{3}[\mathrm{M}+\mathrm{H}]^{+} 460.0461$, found 460.0462 .

IR (film) cm': 3230, 2979, 2933, 2854, 1712, 1666, 1274, 1097, 797.

MP: $53.5-56^{\circ} \mathrm{C}$. 


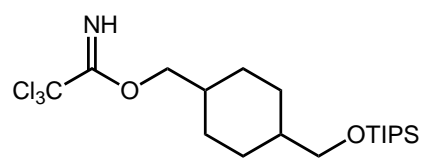

(4-(((triisopropylsilyl)oxy)methyl)cyclohexyl)methyl 2,2,2-trichloroacetimidate (S13)

(4-(((triisopropylsilyl)oxy)methyl)cyclohexyl)methanol $(0.70 \mathrm{~g}, 2.3 \mathrm{mmol})$ was subjected to GP1. Upon completion (monitored by TLC) the reaction was concentrated and loaded directly onto silica gel and purified by flash column chromatography (isocratic: $5: 94: 1$, EtOAc : hexanes : $\left.\mathrm{t}_{3} \mathrm{~N}\right)$ to yield imidate $\mathbf{S} 13(0.97 \mathrm{~g}, 95 \%)$ as a colorless oil.

Rf: 0.43 (10\% EtOAc / hexanes).

${ }^{1} \mathrm{H}$ NMR $\left(600 \mathrm{MHz}, \mathrm{CDCl}_{3}\right): \delta=8.21(\mathrm{bs}, 1 \mathrm{H}), 4.21(\mathrm{~d}, \mathrm{~J}=7.0 \mathrm{~Hz}, 1 \mathrm{H}), 4.10(\mathrm{~d}, \mathrm{~J}=6.5$ $\mathrm{Hz}, 1 \mathrm{H}), 3.57(\mathrm{~d}, \mathrm{~J}=7.0 \mathrm{~Hz}, 1 \mathrm{H}), 3.50(\mathrm{~d}, \mathrm{~J}=6.2 \mathrm{~Hz}, 1 \mathrm{H}), 1.89-1.84(\mathrm{~m}, 3 \mathrm{H}), 1.81-1.75$ $(\mathrm{m}, 1 \mathrm{H}), 1.72-1.67(\mathrm{~m}, 1 \mathrm{H}), 1.58-1.53(\mathrm{~m}, 3 \mathrm{H}), 1.48-1.44(\mathrm{~m}, 2 \mathrm{H}), 1.05(\mathrm{~s}, 18 \mathrm{H})$.

${ }^{13} \mathrm{C}$ NMR (100 MHz, CDCl 3$): \delta=163.32,163.29,91.87,74.74,72.51,68.99,66.60,40.83$, $38.33,37.40,34.56,29.17,29.03,25.66,25.66,25.32,18.19,12.15$.

HRMS (ESI-TOF) m/z: calc'd for $\mathrm{C}_{19} \mathrm{H}_{36} \mathrm{Cl}_{3} \mathrm{NO}_{2} \mathrm{Si}[\mathrm{M}+\mathrm{Na}]^{+}$466.1479, found 466.1464.

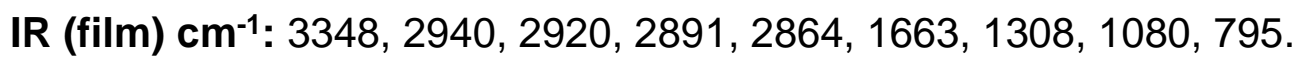
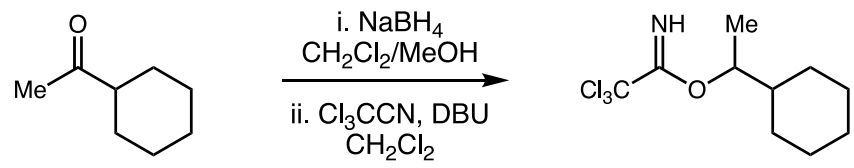

\section{1-cyclohexylethyl 2,2,2-trichloroacetimidate (S14)}

Step 1: Ketone reduction

To a solution of 1-cyclohexylethan-1-one $(0.96 \mathrm{~mL}, 7 \mathrm{mmol})$ in a 1:1 mixture of $\mathrm{CH}_{2} \mathrm{Cl}_{2}$ and $\mathrm{MeOH}(35 \mathrm{~mL})$ was slowly added $\mathrm{NaBH}_{4}$ portionwise $(1.06 \mathrm{~g}, 28.5 \mathrm{mmol})$ at $0^{\circ} \mathrm{C}$. The mixture was warmed to room temperature and stirred for $1 \mathrm{~h}$. Upon completion (monitored by TLC), the reaction was quenched with $\mathrm{H}_{2} \mathrm{O}(35 \mathrm{~mL})$ under vigorous stirring and extracted with $\mathrm{CH}_{2} \mathrm{Cl}_{2}$. The combined organic layer was dried over anhydrous $\mathrm{MgSO}_{4}$ and concentrated in vacuo. The crude mixture of 1-cyclohexylethan-1-ol was carried forward without further purification.

Step 2: Trichloroacetimidate formation

The crude mixture was subjected to GP1 without further purification. Upon completion (monitored by TLC) the reaction was concentrated and loaded directly onto silica gel and 
purified by flash column chromatography (isocratic: $10: 89: 1$, EtOAc : hexane : Et ${ }_{3} \mathrm{~N}$ ) to yield imidate $\mathbf{S} 14$ (0.90 g, 47\% over two steps) as a yellow oil.

Rf: 0.43 (5\% EtOAc/hexanes)

${ }^{1} \mathrm{H}$ NMR (400 MHz, $\left.\mathrm{CDCl}_{3}\right): \delta=8.17(\mathrm{bs}, 1 \mathrm{H}), 4.85$ (quin, $\left.\mathrm{J}=6.3 \mathrm{~Hz}, 1 \mathrm{H}\right), 1.91-1.88(\mathrm{~m}$, $1 \mathrm{H}), 1.79-1.56(\mathrm{~m}, 5 \mathrm{H}), 1.29(\mathrm{~d}, \mathrm{~J}=6.3 \mathrm{~Hz}, 3 \mathrm{H}), 1.27-1.05(\mathrm{~m}, 5 \mathrm{H})$.

${ }^{13} \mathrm{C}$ NMR (100 MHz, $\left.\mathrm{CDCl}_{3}\right): \delta=162.60,92.35,80.25,42.82,28.75,28.45,26.57,26.23$, $26.13,16.02$.

HRMS (ESI-TOF) m/z: calc'd for $\mathrm{C}_{10} \mathrm{H}_{16} \mathrm{Cl}_{3} \mathrm{NO}[\mathrm{M}+\mathrm{Na}]^{+} 294.0190$, found 294.0192 .

IR (neat) cm$^{-1}:$ 3347, 2926, 2853, 1657, 1449, 1075, 791.
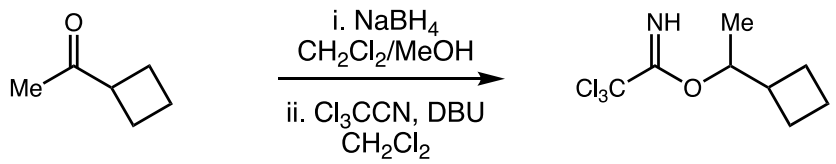

\section{1-cyclobutylethyl 2,2,2-trichloroacetimidate (S15)}

Step 1: Ketone reduction

To a solution of 1-cyclohexylethan-1-one $(0.76 \mathrm{~mL}, 7 \mathrm{mmol})$ in a 1:1 mixture of $\mathrm{CH}_{2} \mathrm{Cl}_{2}$ and $\mathrm{MeOH}(35 \mathrm{~mL})$ was slowly added $\mathrm{NaBH}_{4}$ portionwise $(1.06 \mathrm{~g}, 28.5 \mathrm{mmol})$ at $0^{\circ} \mathrm{C}$. The mixture was warmed to room temperature and stirred for $1 \mathrm{~h}$. Upon completion (monitored by TLC), the reaction was quenched with $\mathrm{H}_{2} \mathrm{O}(35 \mathrm{~mL})$ under vigorous stirring and extracted with $\mathrm{CH}_{2} \mathrm{Cl}_{2}$. The combined organic layer was dried over anhydrous $\mathrm{MgSO}_{4}$ and concentrated in vacuo. The crude mixture of 1-cyclohexylethan-1-ol was carried forward without further purification.

Step 2: Trichloroacetimidate formation

The crude mixture was subjected to GP1 without further purification. Upon completion (monitored by TLC) the reaction was concentrated and loaded directly onto silica gel and purified by flash column chromatography (isocratic: $10: 89: 1$, EtOAc : hexane : $\mathrm{Et}_{3} \mathrm{~N}$ ) to yield imidate $\mathbf{S} 15$ ( $0.85 \mathrm{~g}, 50 \%$ over two steps) as a yellow oil.

Rf: 0.40 (5\% EtOAc/hexanes)

${ }^{1} \mathrm{H}$ NMR (400 MHz, $\mathrm{CDCl}_{3}$ ): $\delta=8.20$ (bs, $1 \mathrm{H}$ ), 5.00 (quint, $\mathrm{J}=6.4 \mathrm{~Hz}, 1 \mathrm{H}$ ), $2.60-2.54$ $(\mathrm{m}, 1 \mathrm{H}), 2.06-1.80(\mathrm{~m}, 6 \mathrm{H}), 1.23(\mathrm{~d}, \mathrm{~J}=6.3 \mathrm{~Hz}, 3 \mathrm{H})$.

${ }^{13} \mathrm{C}$ NMR (100 MHz, $\left.\mathrm{CDCl}_{3}\right): \delta=162.84,92.31,79.26,40.38,24.28,24.25,18.09,16.28$.

HRMS (ESI-TOF) m/z: calc'd for $\mathrm{C}_{8} \mathrm{H}_{12} \mathrm{Cl}_{3} \mathrm{NO}[\mathrm{M}+\mathrm{Na}]^{+} 265.9877$, found 265.9873.

IR (neat) cm-1: 3347, 2977, 2940, 2867, 1658, 1375, 1284, 1066. 
<smiles>CCCC(CCO)CCC(=N)OC(Cl)(Cl)Cl</smiles>

\section{2-propylpentyl 2,2,2-trichloroacetimidate (S16)}

2-propylpentan-1-ol (1.3 g, $10.0 \mathrm{mmol}$ ) was subjected to GP1. Upon completion (monitored by TLC) the reaction was concentrated and loaded directly onto silica gel and purified by flash column chromatography (isocratic: $1: 98: 1$, EtOAc : hexanes : Et ${ }_{3} \mathrm{~N}$ ) to yield imidate S16 $(2.74 \mathrm{~g}, 100 \%)$ as a colorless oil.

Rf: 0.60 (10\% EtOAc / hexanes).

${ }^{1} \mathrm{H}$ NMR (400 MHz, $\left.\mathrm{CDCl}_{3}\right): \delta=8.19(\mathrm{bs}, 1 \mathrm{H}), 4.18(\mathrm{~d}, \mathrm{~J}=5.6 \mathrm{~Hz}, 2 \mathrm{H}), 1.84-1.82(\mathrm{~m}, 1 \mathrm{H})$, $1.41-1.31(\mathrm{~m}, 8 \mathrm{H}), 0.92-0.89(\mathrm{~m}, 6 \mathrm{H})$.

${ }^{13} \mathrm{C}$ NMR (100 MHz, $\left.\mathrm{CDCl}_{3}\right): \delta=163.41,91.88,72.35,36.94,33.67,19.99,14.51$.

HRMS (ESI-TOF) m/z: calc'd for $\mathrm{C}_{10} \mathrm{H}_{18} \mathrm{Cl}_{3} \mathrm{NO}[\mathrm{M}+\mathrm{Na}]^{+} 296.0352$, found 296.0327 .

IR (film) cm$^{-1}:$ 3348, 2957, 2929, 2872, 1662, 1080, 823.

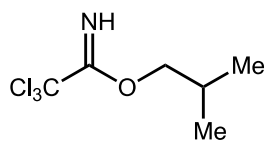

isobutyl 2,2,2-trichloroacetimidate (S17)

2-methylpropan-1-ol $(0.65 \mathrm{~mL}, 7 \mathrm{mmol})$ was subjected to GP1. Upon completion (monitored by TLC) the reaction was concentrated and loaded directly onto silica gel and purified by flash column chromatography (isocratic: $10: 89: 1$, EtOAc : hexane : $\mathrm{Et}_{3} \mathrm{~N}$ ) to yield imidate $\mathbf{S} 17(1.48 \mathrm{~g}, 97 \%)$ as a yellow oil.

Rf: 0.33 (5\% EtOAc/hexanes)

${ }^{1} \mathrm{H}$ NMR (400 MHz, CDCl 3 ): $\delta=8.21(\mathrm{bs}, 1 \mathrm{H}), 4.06(\mathrm{~d}, \mathrm{~J}=6.8 \mathrm{~Hz}, 2 \mathrm{H}$ ), 2.12 (sept, J = 6.7 $\mathrm{Hz}, 1 \mathrm{H}), 1.01(\mathrm{~d}, \mathrm{~J}=6.9 \mathrm{~Hz}, 6 \mathrm{H})$.

${ }^{13} \mathrm{C}$ NMR (100 MHz, $\left.\mathrm{CDCl}_{3}\right): \delta=163.29,91.86,75.64,27.81,19.14$.

HRMS (ESI-TOF) m/z: calc'd for $\mathrm{C}_{6} \mathrm{H}_{10} \mathrm{Cl}_{3} \mathrm{NO}[\mathrm{M}+\mathrm{Na}]^{+} 239.9720$, found 239.9721 .

IR (neat) cm$^{-1}:$ 3347, 2962, 2875, 1662, 1471, 1288, 1076, 1001, 824, 793. 


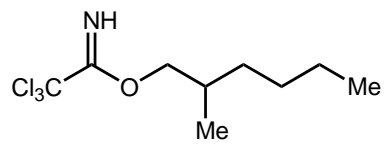

\section{2-methylhexyl 2,2,2-trichloroacetimidate (S18)}

2-methylhexan-1-ol $(0.58 \mathrm{~g}, 5 \mathrm{mmol})$ was subjected to GP1. Upon completion (monitored by TLC) the reaction was concentrated and loaded directly onto silica gel and purified by flash column chromatography (isocratic: $1: 98: 1$, EtOAc : hexanes : Et ${ }_{3} N$ ) to yield imidate $\mathbf{S 1 8}(1.3 \mathrm{~g}, 100 \%)$ as a colorless oil.

Rf: 0.61 (10\% EtOAc / hexanes).

${ }^{1} \mathrm{H}$ NMR (400 MHz, $\left.\mathrm{CDCl}_{3}\right): \delta=8.20$ (bs, $\left.1 \mathrm{H}\right), 4.15(\mathrm{dd}, \mathrm{J}=10.4,5.8 \mathrm{~Hz}, 1 \mathrm{H}), 4.07$ (dd, $\mathrm{J}=10.4,6.7 \mathrm{~Hz}, 1 \mathrm{H}), 2.01-1.90(\mathrm{~m}, 1 \mathrm{H}), 1.50-1.20(\mathrm{~m}, 6 \mathrm{H}), 1.01(\mathrm{~d}, \mathrm{~J}=6.8,3 \mathrm{H}), 0.91-$ $0.88(\mathrm{~m}, 3 \mathrm{H})$.

${ }^{13} \mathrm{C}$ NMR (100 MHz, $\left.\mathrm{CDCl}_{3}\right): \delta=163.35,91.88,74.52,33.04,32.60,29.11,23.00,17.01$, 14.17.

HRMS (ESI-TOF) m/z: calc'd for $\mathrm{C}_{9} \mathrm{H}_{16} \mathrm{Cl}_{3} \mathrm{NO}[\mathrm{M}+\mathrm{Na}]^{+} 282.0195$, found 282.0182.

IR (film) cm-1: 3348, 2957, 2929, 2872, 1662, 1080, 793.
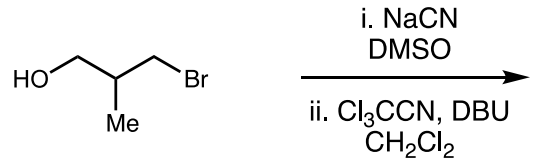

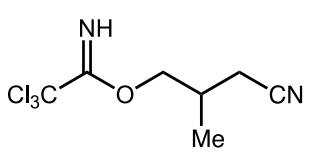

\section{2-cyanopropyl 2,2,2-trichloroacetimidate (S19)}

Step 1: Bromide displacement

To a solution of 3-bromo-2-methylpropan-1-ol $(0.15 \mathrm{~mL}, 1.43 \mathrm{mmol})$ in DMSO $(1.5 \mathrm{~mL})$ was added $\mathrm{NaCN}(77.2 \mathrm{mg}, 1.57 \mathrm{mmol})$. The mixture was warmed to $60^{\circ} \mathrm{C}$ and stirred for overnight. Upon completion (monitored by TLC), the reaction was quenched with $\mathrm{H}_{2} \mathrm{O}$ and extracted with $\mathrm{CH}_{2} \mathrm{Cl}_{2}$. The combined organic layer was washed with $\mathrm{H}_{2} \mathrm{O}$ and brine, dried over anhydrous $\mathrm{MgSO}_{4}$, and concentrated in vacuo. The crude mixture was loaded directly onto silica gel and purified by flash column chromatography (isocratic: $49: 50: 1$, EtOAc : hexane : $\left.\mathrm{Et}_{3} \mathrm{~N}\right)$ to yield 4-hydroxy-3-methylbutanenitrile $(84.1 \mathrm{mg}, 59 \%$ yield) as a yellow oil.

Rf: 0.24 (50\% EtOAc/hexanes) 
Step 2: Trichloroacetimidate formation

4-hydroxy-3-methylbutanenitrile $(0.078 \mathrm{~g}, 0.79 \mathrm{mmol})$ was subjected to GP1. Upon completion (monitored by TLC) the reaction was concentrated and loaded directly onto silica gel and purified by flash column chromatography (isocratic: $20: 79: 1$, EtOAc : hexane : $\left.\mathrm{Et}_{3} \mathrm{~N}\right)$ to yield imidate $\mathbf{S 1 9}(0.19 \mathrm{~g}, 100 \%)$ as a yellow oil.

Rf: 0.23 (20\% EtOAc/hexanes)

${ }^{1} \mathrm{H}$ NMR (400 MHz, $\left.\mathrm{CDCl}_{3}\right): \delta=8.38$ (bs, $\left.1 \mathrm{H}\right), 4.31$ (dd, J = $\left.10.9 \mathrm{~Hz}, 4.5 \mathrm{~Hz}, 1 \mathrm{H}\right), 4.15$ (dd, 10.9, J = 7.3 Hz, 1H), $2.60-2.53(\mathrm{~m}, 1 \mathrm{H}), 2.47-2.38(\mathrm{~m}, 2 \mathrm{H}), 1.21(\mathrm{~d}, \mathrm{~J}=6.8 \mathrm{~Hz}$, $3 \mathrm{H})$.

${ }^{13} \mathrm{C}$ NMR (100 MHz, $\left.\mathrm{CDCl}_{3}\right): \delta=162.58,118.03,91.27,71.61,30.36,21.49,16.15$.

HRMS (ESI-TOF) m/z: calc'd for $\mathrm{C}_{7} \mathrm{H}_{9} \mathrm{Cl}_{3} \mathrm{~N}_{2} \mathrm{O}[\mathrm{M}+\mathrm{Na}]^{+} 264.9673$ found 264.9666 .

IR (neat) cm-1: 3342, 2968, 2246, 1729, 1667, 1616, 1463, 1301.

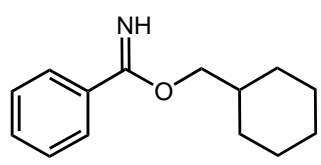

\section{cyclohexylmethyl benzimidate (S20)}

2-methylhexan-1-ol $(1.14 \mathrm{~g}, 1.23 \mathrm{~mL}, 10.0 \mathrm{mmol})$ and benzonitrile $(1.1 \mathrm{~g}, 1.13 \mathrm{~mL} 11.0$ $\mathrm{mmol}$ ) was subjected to GP2. Upon completion, the reaction was concentrated and loaded directly onto silica gel and purified by flash column chromatography (isocratic: 10 : 89 : 1, EtOAc : hexanes : Et3 $\mathrm{N})$ to yield imidate $\mathbf{S} 20(1.91 \mathrm{~g}, 80 \%)$ as a colorless oil.

Rf: 0.50 (20\% EtOAc / hexanes).

${ }^{1} \mathrm{H}$ NMR (400 MHz, $\left.\mathrm{CDCl}_{3}\right): \delta=7.75(\mathrm{~d}, \mathrm{~J}=7.0 \mathrm{~Hz}, 2 \mathrm{H}), 7.47-7.38(\mathrm{~m}, 3 \mathrm{H}), 4.07(\mathrm{~d}, \mathrm{~J}=$ $5.6 \mathrm{~Hz}, 2 \mathrm{H}), 1.90-1.68(\mathrm{~m}, 6 \mathrm{H}), 1.36-1.05(\mathrm{~m}, 5 \mathrm{H})$.

${ }^{13} \mathrm{C}$ NMR (100 MHz, $\left.\mathrm{CDCl}_{3}\right): \delta=168.17,133.15,130.89,128.53,126.80,71.41,37.38$, $30.07,26.61,25.94$.

HRMS (ESI-TOF) m/z: calc'd for $\mathrm{C}_{14} \mathrm{H}_{19} \mathrm{NO}[\mathrm{M}+\mathrm{Na}]^{+} 240.1364$, found 240.1373 .

IR (film) cm': 3336, 3087, 3026, 2922, 2831, 1631, 1494, 1065. 


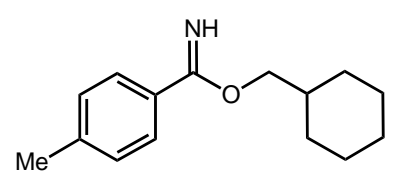

\section{cyclohexylmethyl 4-methylbenzimidate (S21)}

2-methylhexan-1-ol (1.14 g, $1.23 \mathrm{~mL}, 10.0 \mathrm{mmol})$ and 4-methylbenzonitrile $(1.29 \mathrm{~g}, 1.31$ $\mathrm{mL}, 11.0 \mathrm{mmol}$ ) was subjected to GP2. Upon completion, the reaction was concentrated and loaded directly onto silica gel and purified by flash column chromatography (isocratic: $10: 89: 1$, EtOAc : hexanes : $\left.\mathrm{Et}_{3} \mathrm{~N}\right)$ to yield imidate $\mathbf{S 2 1}(1.57 \mathrm{~g}, 68 \%)$ as a colorless oil.

Rf: 0.38 (20\% EtOAc/hexanes).

${ }^{1} \mathrm{H}$ NMR $\left(400 \mathrm{MHz}, \mathrm{CDCl}_{3}\right): \delta=7.65-7.63(\mathrm{~m}, 3 \mathrm{H}), 7.22-7.20(\mathrm{~m}, 2 \mathrm{H}), 4.05(\mathrm{~d}, \mathrm{~J}=6.1 \mathrm{~Hz}$, $2 \mathrm{H}), 2.39(\mathrm{~s}, 3 \mathrm{H}), 1.89-1.69(\mathrm{~m}, 6 \mathrm{H}), 1.36-1.08(\mathrm{~m}, 5 \mathrm{H})$.

${ }^{13} \mathrm{C}$ NMR (100 MHz, $\left.\mathrm{CDCl}_{3}\right): \delta=168.10,141.19,130.34,129.22,126.77,71.32,37.40$, $30.10,26.64,25.96,21.50$.

HRMS (ESI-TOF) m/z: calc'd for $\mathrm{C}_{15} \mathrm{H}_{21} \mathrm{NO}[\mathrm{M}+\mathrm{H}]^{+} 232.1701$, found 232.1699.

IR (film) cm$^{-1}:$ 3306, 3032, 2922, 2851, 1629, 1328, 1064, 814, 729.

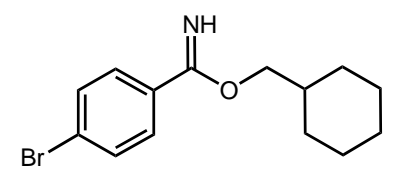

\section{cyclohexylmethyl 4-bromobenzimidate (S22)}

2-methylhexan-1-ol (0.46 g, $0.49 \mathrm{~mL}, 4.0 \mathrm{mmol})$ and 4-bromobenzonitrile $(0.73 \mathrm{~g}, 4.0$ $\mathrm{mmol}$ ) was subjected to GP2. Upon completion, the reaction was concentrated and loaded directly onto silica gel and purified by flash column chromatography (isocratic: 10 : 89 : 1, EtOAc : hexanes : Et $\left.{ }_{3} \mathrm{~N}\right)$ to yield imidate $\mathbf{S 2 2}(0.89 \mathrm{~g}, 75 \%)$ as a colorless oil.

Rf: 0.50 (20\% EtOAc / hexanes).

${ }^{1} \mathrm{H}$ NMR (400 MHz, $\left.\mathrm{CDCl}_{3}\right): \delta=7.65(\mathrm{~d}, \mathrm{~J}=8.6 \mathrm{~Hz}, 2 \mathrm{H}), 7.57-7.54(\mathrm{~m}, 2 \mathrm{H}), 4.05(\mathrm{~d}, \mathrm{~J}=$ $6.0 \mathrm{~Hz}, 2 \mathrm{H}), 1.89-1.20(\mathrm{~m}, 6 \mathrm{H}), 1.37-1.20(\mathrm{~m}, 3 \mathrm{H}), 1.12-1.06(\mathrm{~m}, 2 \mathrm{H})$.

${ }^{13} \mathrm{C}$ NMR (100 MHz, $\left.\mathrm{CDCl}_{3}\right): \delta=166.74,132.03,131.74,128.52,125.53,71.45,37.31$, $30.05,26.57,25.90$.

HRMS (ESI-TOF) m/z: calc'd for $\mathrm{C}_{14} \mathrm{H}_{18} \mathrm{BrNO}[\mathrm{M}+\mathrm{H}]^{+}$296.0650, found 296.0680 .

IR (film) cm$^{-1}:$ 3306, 3041, 2921, 2850, 1630, 1067, 816. 
<smiles>N=C(OCC1CCCCC1)c1ccc(C(F)(F)F)cc1</smiles>

\section{cyclohexylmethyl 4-(trifluoromethyl)benzimidate (S23)}

2-methylhexan-1-ol $(0.46 \mathrm{~g}, 0.49 \mathrm{~mL}, 4.0 \mathrm{mmol})$ and 4-(trifluoromethyl)benzonitrile (0.68 $\mathrm{g}, 4.0 \mathrm{mmol})$ was subjected to GP2. Upon completion, the reaction was concentrated and loaded directly onto silica gel and purified by flash column chromatography (isocratic: $10: 89: 1$, EtOAc : hexanes : Et ${ }_{3} N$ ) to yield imidate $\mathbf{S 2 3}(0.59$ $\mathrm{g}, 52 \%)$ as a colorless oil.

Rf: 0.48 (20\% EtOAc / hexanes).

${ }^{1} \mathrm{H}$ NMR $\left(600 \mathrm{MHz}, \mathrm{CDCl}_{3}\right): \delta=7.86(\mathrm{bs}, 2 \mathrm{H}), 7.65(\mathrm{td} \mathrm{J}=29.9 \mathrm{~Hz}, 8.3 \mathrm{~Hz}, 2 \mathrm{H}), 4.08(\mathrm{bs}$, $2 \mathrm{H}), 1.87-1.69(\mathrm{~m}, 6 \mathrm{H}), 1.35-1.20(\mathrm{~m}, 3 \mathrm{H}), 1.13-1.07(\mathrm{~m}, 2 \mathrm{H})$.

${ }^{13} \mathrm{C} \mathrm{NMR}\left(150 \mathrm{MHz}, \mathrm{CDCl}_{3}\right): \delta=167.27,136.47,132.73$ (q, $\left.{ }^{2} \mathrm{~J}_{\mathrm{CF}}=32.6 \mathrm{~Hz}\right), 127.30$, $125.58,123.91\left(\mathrm{q},{ }^{1} \mathrm{JCF}=274.4 \mathrm{~Hz}\right), 71.92,37.33,30.06,26.57,25.91$.

${ }^{19} \mathrm{~F}$ NMR (564 MHz, $\left.\mathrm{CDCl}_{3}\right): \delta=-62.94$.

HRMS (ESI-TOF) m/z: calc'd for $\mathrm{C}_{15} \mathrm{H}_{18} \mathrm{~F}_{3} \mathrm{NO}[\mathrm{M}+\mathrm{H}]^{+} 286.1382$, found 286.1410 .

IR (film) cm'-1: 3364, 3028, 2970, 1644, 1320, 1016, 953.<smiles>N=C(OCC1CCCCC1)c1ccc(-c2ccccc2)cc1</smiles>

\section{cyclohexylmethyl [1,1'-biphenyl]-4-carbimidate (S24)}

2-methylhexan-1-ol $(0.46 \mathrm{~g}, 0.49 \mathrm{~mL}, 4.0 \mathrm{mmol})$ and [1,1'-biphenyl]-4-carbonitrile $(0.72$ $\mathrm{g}, 4.0 \mathrm{mmol}$ ) was subjected to GP2. Upon completion, the reaction was concentrated and loaded directly onto silica gel and purified by flash column chromatography (isocratic: $10: 89: 1$, EtOAc : hexanes : $\left.\mathrm{Et}_{3} \mathrm{~N}\right)$ to yield imidate $\mathbf{S} 24(0.94 \mathrm{~g}, 80 \%)$ as a colorless oil.

Rf: 0.41 (20\% EtOAc / hexanes).

${ }^{1} \mathrm{H}$ NMR (400 MHz, $\left.\mathrm{CDCl}_{3}\right): \delta=7.82(\mathrm{bs}, 2 \mathrm{H}), 7.65-7.60(\mathrm{~m}, 4 \mathrm{H}), 7.48-7.44(\mathrm{~m}, 2 \mathrm{H}), 7.40-$ $7.36(\mathrm{~m}, 1 \mathrm{H}), 4.11$ (bs, 2H), 1.93-1.71 (m, 6H), 1.39-1.08 (m, 5H). 
${ }^{13} \mathrm{C}$ NMR (100 MHz, $\left.\mathrm{CDCl}_{3}\right): \delta=168.21,143.68,140.22,131.79,128.98,127.96,127.24$, $127.19,71.54,37.37,30.06,26.59,25.92$.

HRMS (ESI-TOF) m/z: calc'd for $\mathrm{C}_{20} \mathrm{H}_{23} \mathrm{NO}[\mathrm{M}+\mathrm{H}]^{+} 294.1858$, found 294.1878.

IR (film) cm': 3349, 3029, 2976, 2924, 1628, 1073, 736.

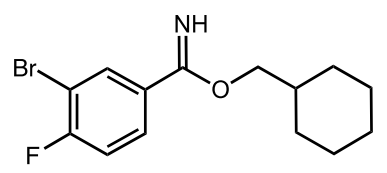

\section{cyclohexylmethyl 3-bromo-4-fluorobenzimidate (S25)}

2-methylhexan-1-ol $(1.14 \mathrm{~g}, 1.23 \mathrm{~mL}, 10.0 \mathrm{mmol})$ and 3-bromo-4-fluorobenzonitrile (2.0 $\mathrm{g}, 10.0 \mathrm{mmol}$ ) was subjected to GP2. Upon completion, the reaction was concentrated and loaded directly onto silica gel and purified by flash column chromatography (isocratic: $10: 89: 1$, EtOAc : hexanes : $\left.\mathrm{Et}_{3} \mathrm{~N}\right)$ to yield imidate $\mathbf{S} 25(2.04 \mathrm{~g}, 65 \%)$ as a colorless oil.

Rf: 0.33 (20\% EtOAc/hexanes).

${ }^{1} \mathrm{H}$ NMR (400 MHz, $\left.\mathrm{CDCl}_{3}\right)$ : $\delta=7.95-7.94(\mathrm{~m}, 1 \mathrm{H}), 7.67-7.67(\mathrm{~m}, 1 \mathrm{H}), 7.46(\mathrm{bs}, 1 \mathrm{H}), 7.09$ (t, J = 8.4 Hz, 1H), $3.97(\mathrm{~d}, \mathrm{~J}=5.8 \mathrm{~Hz}, 2 \mathrm{H}), 1.83-1.79(\mathrm{~m}, 6 \mathrm{H}), 1.31-0.98(\mathrm{~m}, 5 \mathrm{H})$.

${ }^{13} \mathrm{C}$ NMR (100 MHz, CDCl 3$): \delta=165.88,160.59$ (d, 252.1 Hz), 132.39, 130.61, 127.73, 116.31 (d, $22.9 \mathrm{~Hz}$ ), 109.22 (d, $21.7 \mathrm{~Hz}$ ), 71.72, 37.14, 29.94, 26.46, 25.77.

${ }^{19} \mathrm{~F}$ NMR (367 MHz, $\left.\mathrm{CDCl}_{3}\right): \delta=-103.65$.

HRMS (ESI-TOF) m/z: calc'd for $\mathrm{C}_{14} \mathrm{H}_{17} \mathrm{BrFNO}[\mathrm{M}+\mathrm{H}]^{+} 314.0556$, found 314.0538 .

IR (film) cm$^{-1}:$ 3290, 3041, 2923, 2851, 1634, 1322, 1070, 730.

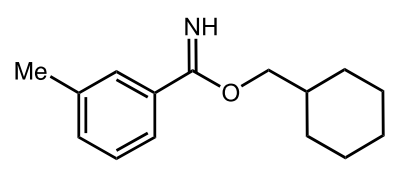

\section{cyclohexylmethyl 3-methylbenzimidate (S26)}

2-methylhexan-1-ol (1.14 g, $1.23 \mathrm{~mL}, 10.0 \mathrm{mmol})$ and 3-methylbenzonitrile (1.29 g, 1.32 $\mathrm{mL}, 11.0 \mathrm{mmol}$ ) was subjected to GP2. Upon completion, the reaction was concentrated and loaded directly onto silica gel and purified by flash column chromatography (isocratic: $5: 94$ : 1, EtOAc : hexanes : Et $\left.{ }_{3} \mathrm{~N}\right)$ to yield imidate $\mathbf{S 2 6}(1.25 \mathrm{~g}, 54 \%)$ as a colorless oil. 
Rf: 0.39 (20\% EtOAc/hexanes).

${ }^{1} \mathrm{H}$ NMR (600 MHz, $\left.\mathrm{CDCl}_{3}\right): \delta=7.96(\mathrm{~s}, 1 \mathrm{H}), 7.61-7.57(\mathrm{~m}, 2 \mathrm{H}), 7.31-7.26(\mathrm{~m}, 2 \mathrm{H}), 4.00$ $(\mathrm{d}, \mathrm{J}=6.4 \mathrm{~Hz}, 2 \mathrm{H}), 2.36(\mathrm{~s}, 3 \mathrm{H}), 1.84-1.65(\mathrm{~m}, 6 \mathrm{H}), 1.33-1.26(\mathrm{~m}, 2 \mathrm{H}), 1.24-1.14(\mathrm{~m}, 1 \mathrm{H})$, 1.11-1.04 (m, 2H).

${ }^{13} \mathrm{C}$ NMR (150 MHz, $\left.\mathrm{CDCl}_{3}\right): \delta=168.13,139.77,134.66,132.88,129.87,128.84,125.40$, 72.24, 38.76, 31.31, 27.90, 27.24, 22.10.

HRMS (ESI-TOF) m/z: calc'd for $\mathrm{C}_{15} \mathrm{H}_{21} \mathrm{NO}[\mathrm{M}+\mathrm{H}]^{+} 232.1701$, found 232.1707.

IR (film) cm-1: 3335, 3044, 2923, 2852, 1632, 1328, 1068, 795.

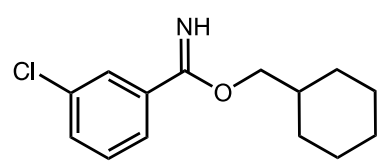

\section{cyclohexylmethyl 3-chlorobenzimidate (S27)}

2-methylhexan-1-ol (1.14 g, $1.23 \mathrm{~mL}, 10.0 \mathrm{mmol})$ and 3-chlorobenzonitrile $(1.51 \mathrm{~g}, 11.0$ $\mathrm{mmol}$ ) was subjected to GP2. Upon completion, the reaction was concentrated and loaded directly onto silica gel and purified by flash column chromatography (isocratic: 10 : 89 : 1, EtOAc : hexanes : Et $\left.{ }_{3} \mathrm{~N}\right)$ to yield imidate $\mathbf{S 2 7}(1.11 \mathrm{~g}, 44 \%)$ as a colorless oil.

Rf: 0.36 (20\% EtOAc/hexanes).

${ }^{1} \mathrm{H}$ NMR $\left(600 \mathrm{MHz}, \mathrm{CD}_{3} \mathrm{CN}\right): \delta=8.08(\mathrm{bs}, 1 \mathrm{H}), 7.79-7.78(\mathrm{~m}, 1 \mathrm{H}), 7.72-7.71(\mathrm{~m}, 1 \mathrm{H})$, 7.48-7.46 $(\mathrm{m}, 1 \mathrm{H}), 7.41-7.38(\mathrm{~m}, 1 \mathrm{H}), 3.98(\mathrm{~d}, \mathrm{~J}=6.3 \mathrm{~Hz}, 2 \mathrm{H}), 1.83-1.65(\mathrm{~m}, 6 \mathrm{H}), 1.32-$ $1.25(\mathrm{~m}, 2 \mathrm{H}), 1.23-1.16(\mathrm{~m}, 1 \mathrm{H}), 1.10-1.04(\mathrm{~m}, 2 \mathrm{H})$.

${ }^{13} \mathrm{C}$ NMR (100 MHz, $\left.\mathrm{CD}_{3} \mathrm{CN}\right): \delta=166.00,136.40,135.37,131.99,131.49,128.25$, $126.73,72.78,38.48,31.07,27.68,27.02$.

HRMS (ESI-TOF) m/z: calc'd for $\mathrm{C}_{14} \mathrm{H}_{18} \mathrm{CINO}[\mathrm{M}+\mathrm{H}]^{+} 252.1155$, found 252.1149.

IR (film) cm-1: 3336, 3047, 2925, 2852, 1633, 1326, 1069, 730. 


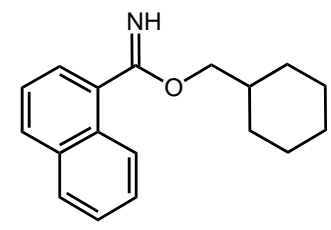

cyclohexylmethyl 1-naphthimidate (S28)

2-methylhexan-1-ol (1.14 g, $1.23 \mathrm{~mL}, 10.0 \mathrm{mmol})$ and 1-naphthonitrile (1.68 g, 11.0 $\mathrm{mmol}$ ) was subjected to GP2. Upon completion, the reaction was concentrated and loaded directly onto silica gel and purified by flash column chromatography (isocratic: 10 : 89 : 1, EtOAc : hexanes : $\left.\mathrm{Et}_{3} \mathrm{~N}\right)$ to yield imidate $\mathbf{S 2 8}(0.67 \mathrm{~g}, 25 \%)$ as a colorless oil.

Rf: 0.39 (20\% EtOAc/hexanes).

${ }^{1} \mathrm{H}$ NMR $\left(600 \mathrm{MHz}, \mathrm{CDCl}_{3}\right): \delta=8.10(\mathrm{bd}, 8.3 \mathrm{~Hz}, 1 \mathrm{H}), 7.89-7.86(\mathrm{~m}, 2 \mathrm{H}), 7.60-7.45(\mathrm{~m}$, $5 \mathrm{H}), 4.23(\mathrm{~d}, \mathrm{~J}=6.4 \mathrm{~Hz}, 2 \mathrm{H}), 1.91-1.83(\mathrm{~m}, 3 \mathrm{H}), 1.79-1.76(\mathrm{~m}, 2 \mathrm{H}), 1.71-1.69(\mathrm{~m}, 1 \mathrm{H})$, $1.33-1.20(\mathrm{~m}, 3 \mathrm{H}), 1.16-1.09(\mathrm{~m}, 2 \mathrm{H})$.

${ }^{13} \mathrm{C}$ NMR (150 MHz, $\left.\mathrm{CDCl}_{3}\right): \delta=170.31,134.02,133.72,130.08,129.91,128.51,126.97$, $126.27,125.50,125.23,125.02,71.85,37.34,30.09,26.56,25.89$.

HRMS (ESI-TOF) m/z: calc'd for $\mathrm{C}_{18} \mathrm{H}_{21} \mathrm{NO}[\mathrm{M}+\mathrm{H}]^{+} 268.1701$, found 268.1695 .

IR (film) cm-1: 3335, 3044, 2923, 2852, 1632, 1328, 1068, 731.

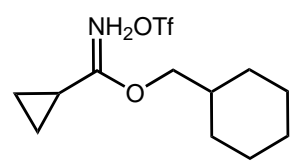

cyclohexylmethyl cyclopropanecarbimidate triflate (S29)

2-methylhexan-1-ol $(1.14 \mathrm{~g}, 1.23 \mathrm{~mL}, 10.0 \mathrm{mmol})$ and cyclopropanecarbonitrile $(0.74 \mathrm{~g}$, $0.81 \mathrm{~mL}, 11.0 \mathrm{mmol}$ ) was subjected to GP2(a) to yield imidate salt S29 (1.01 g, 30\%) as a white solid.

${ }^{1} \mathrm{H}$ NMR (600 MHz, CD $\left.3 \mathrm{CN}\right): \delta=9.51$ (bs, $\left.1 \mathrm{H}\right), 8.64(\mathrm{bs}, 1 \mathrm{H}), 4.05(\mathrm{~d}, \mathrm{~J}=6.4 \mathrm{~Hz}, 2 \mathrm{H})$, 2.02-1.97 (m, $1 \mathrm{H}), 1.83-1.76(\mathrm{~m}, 1 \mathrm{H}), 1.75-1.65(\mathrm{~m}, 5 \mathrm{H}), 1.32-1.24(\mathrm{~m}, 6 \mathrm{H}), 1.23-1.15(\mathrm{~m}$, $1 \mathrm{H}), 1.06-0.99(\mathrm{~m}, 2 \mathrm{H})$.

${ }^{13} \mathrm{C}$ NMR (150 MHz, CD $\left.{ }_{3} \mathrm{CN}\right): \delta=182.93,78.79,37.53,29.89,27.14,26.49,14.38,12.29$.

${ }^{19} \mathrm{~F}$ NMR (564 MHz, $\left.\mathrm{CD}_{3} \mathrm{CN}\right): \delta=-79.39$. 
HRMS (ESI-TOF) m/z: calc'd for $\mathrm{C}_{11} \mathrm{H}_{19} \mathrm{NO}$ [M-OTf]+ 182.1545 , found 182.1533.

IR (film) cm': 3256, 3098, 2926, 2853, 1704, 1224, 1026, 633.

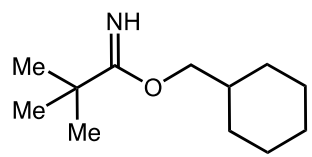

\section{cyclohexylmethyl pivalimidate (S30)}

2-methylhexan-1-ol $(1.14 \mathrm{~g}, 1.23 \mathrm{~mL}, 10.0 \mathrm{mmol})$ and pivalonitrile $(0.83 \mathrm{~g}, 1.22 \mathrm{~mL}, 11.0$ $\mathrm{mmol}$ ) was subjected to GP2. Upon completion, the reaction was concentrated and loaded directly onto silica gel and purified by flash column chromatography (isocratic: 10 : 89 : 1, EtOAc : hexanes : Et $\left.{ }_{3} \mathrm{~N}\right)$ to yield imidate $\mathbf{S} 30(0.69 \mathrm{~g}, 35 \%)$ as a colorless oil.

Rf: 0.36 (20\% EtOAc/hexanes).

${ }^{1} \mathrm{H}$ NMR $\left(400 \mathrm{MHz}, \mathrm{CDCl}_{3}\right): \delta=6.71(\mathrm{bs}, 1 \mathrm{H}), 3.73(\mathrm{~d}, \mathrm{~J}=6.4 \mathrm{~Hz}, 2 \mathrm{H}), 1.70-1.55(\mathrm{~m}, 7 \mathrm{H})$, 1.23-1.09 (m, 2H), $1.07(\mathrm{~s}, 9 \mathrm{H}), 0.97-0.88(\mathrm{~m}, 2 \mathrm{H})$.

${ }^{13} \mathrm{C}$ NMR $\left(100 \mathrm{MHz}, \mathrm{CDCl}_{3}\right): \delta=178.79,70.71,37.84,37.21,29.82,27.67,26.48,25.80$.

HRMS (ESI-TOF) m/z: calc'd for $\mathrm{C}_{12} \mathrm{H}_{23} \mathrm{NO}[\mathrm{M}+\mathrm{H}]^{+} 198.1858$, found 198.1871 .

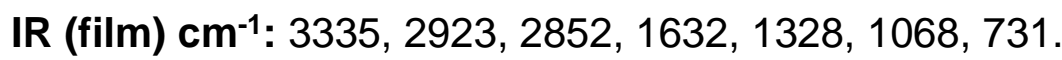<smiles>N=C(OCCCC(=O)c1ccccc1)C(Cl)(Cl)Cl</smiles>

\section{4-oxo-4-phenylbutyl 2,2,2-trichloroacetimidate (33)}

4-hydroxy-1-phenylbutan-1-one ${ }^{1}$ (1.42 g, 8.6 mmol) was subjected to GP1. Upon completion (monitored by TLC) the reaction was concentrated and loaded directly onto silica gel and purified by flash column chromatography (isocratic: $0: 99: 1$, EtOAc : hexane : $\left.\mathrm{Et}_{3} \mathrm{~N}\right)$ to yield imidate $33(2.24 \mathrm{~g}, 90 \%)$ as a yellow solid.

Rf: 0.36 (20\% EtOAc/hexanes)

${ }^{1} \mathrm{H}$ NMR $\left(400 \mathrm{MHz}, \mathrm{CDCl}_{3}\right): \delta=8.30$ (bs, $\left.1 \mathrm{H}\right), 7.99-7.96(\mathrm{~m}, 2 \mathrm{H}), 7.59-7.55(\mathrm{~m}, 1 \mathrm{H})$, $7.48-7.45(\mathrm{~m}, 2 \mathrm{H}), 4.42(\mathrm{t}, \mathrm{J}=6.2 \mathrm{~Hz}, 2 \mathrm{H}), 3.17(\mathrm{t}, \mathrm{J}=7.2 \mathrm{~Hz}, 2 \mathrm{H}), 2.25$ (quint, $\mathrm{J}=$ $6.7 \mathrm{~Hz}, 2 \mathrm{H})$.

${ }^{1}$ Murphy, S. K.; Dong, V. M. J. Am. Chem. Soc. 2013, 135, 5553. 
${ }^{13} \mathrm{C}$ NMR (100 MHz, $\left.\mathrm{CDCl}_{3}\right): \delta=199.18,162.95,136.99,133.28,128.78,128.16$, 91.68, 68.83, 34.79, 23.10.

HRMS (ESI-TOF) m/z: calc'd for $\mathrm{C}_{12} \mathrm{H}_{12} \mathrm{Cl}_{3} \mathrm{NO}_{2}[\mathrm{M}+\mathrm{Na}]^{+} 329.9826$, found 329.9825 .

IR (neat) cm$^{-1}:$ 3341, 2978, 2942, 2906, 1676, 1662, 1595, 1580, 1474.

MP: $51.3^{\circ} \mathrm{C}-54.2^{\circ} \mathrm{C}$.<smiles>N=C(OCCCC(=O)OCc1ccccc1)C(Cl)(Cl)Cl</smiles>

benzyl 4-(2,2,2-trichloro-1-iminoethoxy)butanoate (35)

Benzyl 4-hydroxybutanoate ${ }^{2}(3.69 \mathrm{~g}, 19 \mathrm{mmol}$ ) was subjected to GP1. Upon completion (monitored by TLC) the reaction was concentrated and loaded directly onto silica gel and purified by flash column chromatography (isocratic: $20: 79: 1$, EtOAc : hexane : $\left.\mathrm{Et}_{3} \mathrm{~N}\right)$ to yield imidate $35(5.17 \mathrm{~g}, 80 \%)$ as a yellow oil.

Rf: 0.63 (25\% EtOAc/hexanes)

${ }^{1} \mathrm{H}$ NMR $\left(400 \mathrm{MHz}, \mathrm{CDCl}_{3}\right): \delta=8.29(\mathrm{bs}, 1 \mathrm{H}), 7.39-7.30(\mathrm{~m}, 5 \mathrm{H}), 5.14(\mathrm{~s}, 2 \mathrm{H}), 4.34(\mathrm{t}$, $\mathrm{J}=6.2 \mathrm{~Hz}, 2 \mathrm{H}), 2.55(\mathrm{t}, \mathrm{J}=7.4 \mathrm{~Hz}, 2 \mathrm{H}), 2.18-2.11(\mathrm{~m}, 2 \mathrm{H})$.

${ }^{13} \mathrm{C}$ NMR (100 MHz, $\left.\mathrm{CDCl}_{3}\right): \delta=172.75,162.91,136.02,128.73,128.42,128.38$, $91.56,68.43,66.54,30.77,23.89$.

HRMS (ESI-TOF) m/z: calc'd for $\mathrm{C}_{13} \mathrm{H}_{14} \mathrm{Cl}_{3} \mathrm{NO}_{3}[\mathrm{M}+\mathrm{Na}]^{+} 359.9931$, found 359.9924 .

IR (neat) cm$^{-1}:$ 3340, 2958, 1732, 1663, 1498, 1455, 1304, 1165, 1075.<smiles>N=C(Cl)OCCCBr</smiles>

\section{3-bromopropyl 2,2,2-trichloroacetimidate (37)}

3-bromopropan-1-ol (0.90 mL, $10 \mathrm{mmol})$ was subjected to GP1. Upon completion (monitored by TLC) the reaction was concentrated and loaded directly onto silica gel and purified by flash column chromatography (isocratic: $10: 89: 1$, EtOAc : hexane : $\left.\mathrm{Et}_{3} \mathrm{~N}\right)$ to yield imidate $37(2.07 \mathrm{~g}, 73 \%)$ as a colorless oil.

Rf: 0.42 (5\% EtOAc/hexanes)

${ }^{2}$ Weber, A. E.; Halgren, T. A.; Doyle, J. J.; Lynch, R. J.; Siegl, P. K. S.; Parsons, W. H.; Greenlee, W. J.; Patchett, A. A. J. Med. Chem. 1991, 34, 2962. 
${ }^{1} \mathrm{H}$ NMR $\left(400 \mathrm{MHz}, \mathrm{CDCl}_{3}\right): \delta=8.33(\mathrm{bs}, 1 \mathrm{H}), 4.45(\mathrm{t}, \mathrm{J}=5.9 \mathrm{~Hz}, 2 \mathrm{H}), 3.70(\mathrm{t}, \mathrm{J}=6.4$ $\mathrm{Hz}, 2 \mathrm{H}), 2.27-2.21(\mathrm{~m}, 2 \mathrm{H})$.

${ }^{13} \mathrm{C}$ NMR (100 MHz, $\left.\mathrm{CDCl}_{3}\right): \delta=162.78,91.48,66.01,41.19,31.44$.

HRMS (ESI-TOF) m/z: calc'd for $\mathrm{C}_{7} \mathrm{H}_{12} \mathrm{Cl}_{3} \mathrm{NO}[\mathrm{M}+\mathrm{Na}]^{+}$302.8596, found 302.8591 .

IR (neat) cm$^{-1}:$ 3335, 2968, 1070, 760, 631.

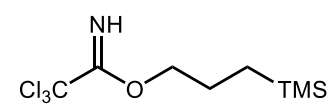

\section{3-(trimethylsilyl)propyl 2,2,2-trichloroacetimidate (39)}

3-(trimethylsilyl)propan-1-ol $(1.11 \mathrm{~mL}, 7 \mathrm{mmol})$ was subjected to GP1. Upon completion (monitored by TLC) the reaction was concentrated and loaded directly onto silica gel and purified by flash column chromatography (isocratic: $10: 89: 1$, EtOAc : hexane : Et ${ }_{3} \mathrm{~N}$ ) to yield imidate $39(1.95 \mathrm{~g}, 100 \%)$ as a colorless oil.

Rf: 0.46 (5\% EtOAc/hexanes)

${ }^{1} \mathrm{H}$ NMR $\left(400 \mathrm{MHz}, \mathrm{CDCl}_{3}\right): \delta=8.22(\mathrm{bs}, 1 \mathrm{H}), 4.25(\mathrm{~d}, \mathrm{~J}=6.9 \mathrm{~Hz}, 2 \mathrm{H}), 1.81-1.73(\mathrm{~m}$, $2 \mathrm{H}), 0.61-0.53(\mathrm{~m}, 2 \mathrm{H}), 0.01(\mathrm{~s}, 9 \mathrm{H})$.

${ }^{13} \mathrm{C}$ NMR $\left(100 \mathrm{MHz}, \mathrm{CDCl}_{3}\right): \delta=163.20,91.86,72.20,23.03,12.44,-1.63$.

HRMS (ESI-TOF) m/z: calc'd for $\mathrm{C}_{8} \mathrm{H}_{16} \mathrm{Cl}_{3} \mathrm{NOSi}[\mathrm{M}+\mathrm{Na}]^{+}$297.9959, found 297.9952 .

IR (neat) cm$^{-1}:$ 3348, 2953, 1662, 1386, 1291, 1248, 1080.

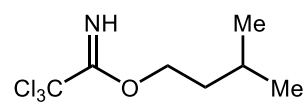

isopentyl 2,2,2-trichloroacetimidate (41)

3-methylbutan-1-ol ( $0.76 \mathrm{~mL}, 7 \mathrm{mmol})$ was subjected to GP1. Upon completion (monitored by TLC) the reaction was concentrated and loaded directly onto silica gel and purified by flash column chromatography (isocratic: $10: 89: 1$, EtOAc : hexane : Et $3 \mathrm{~N}$ ) to yield imidate 41 (1.53 g, 94\%) as a colorless oil.

Rf: 0.38 (5\% EtOAc/hexanes)

${ }^{1} \mathrm{H}$ NMR $\left(400 \mathrm{MHz}, \mathrm{CDCl}_{3}\right): \delta=8.22(\mathrm{bs}, 1 \mathrm{H}), 4.32(\mathrm{~d}, \mathrm{~J}=6.7 \mathrm{~Hz}, 2 \mathrm{H}), 1.85-1.75(\mathrm{~m}$, $1 \mathrm{H}), 1.67(\mathrm{q}, \mathrm{J}=6.7 \mathrm{~Hz}, 2 \mathrm{H}), 0.96(\mathrm{~d}, \mathrm{~J}=6.7 \mathrm{~Hz}, 6 \mathrm{H})$. 
${ }^{13} \mathrm{C}$ NMR (100 MHz, $\left.\mathrm{CDCl}_{3}\right): \delta=163.25,91.82,68.37,37.10,25.23,22.62$.

HRMS (ESI-TOF) m/z: calc'd for $\mathrm{C}_{7} \mathrm{H}_{12} \mathrm{Cl}_{3} \mathrm{NO}[\mathrm{M}+\mathrm{Na}]^{+}$253.9877, found 253.9878.

IR (neat) cm$^{-1}: 3347,2958,2871,1662,1465,1308,1078,794$. 


\section{Amino-halogenation Optimization}

Imidate (1) $(51.7 \mathrm{mg}, 0.2 \mathrm{mmol}$ ) was subjected to GP3. Upon completion, the combined organic layer was concentrated and the yields and diastereomeric ratios were determined by ${ }^{1} \mathrm{H}-\mathrm{NMR}$ using dichloroethane as an internal standard.<smiles>N=C(OCC1CCCCC1)C(Cl)(Cl)Cl</smiles>
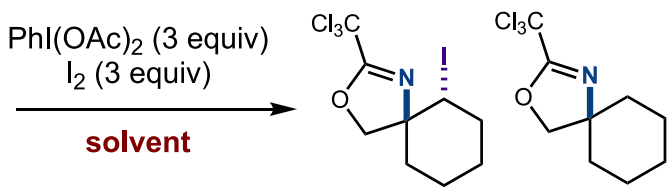

A

B

Table S1: Solvent Effects

\begin{tabular}{|c|c|c|c|}
\hline Entry & Solvent & A & B \\
\hline 1 & $\mathrm{MeCN}$ & $30 \%, 20: 1 d r$ & $28 \%$ \\
\hline 2 & $\mathrm{CH}_{2} \mathrm{Cl}_{2}$ & $43 \%, 20: 1 \mathrm{dr}$ & $15 \%$ \\
\hline 3 & $\mathrm{PhCF}_{3}$ & $55 \%, 3: 1 \mathrm{dr}$ & $8 \%$ \\
\hline 4 & HFIP & $0 \%$ & $38 \%$ \\
\hline 5 & HFIP: $\mathrm{CH}_{2} \mathrm{Cl}_{2}(3: 1)$ & $0 \%$ & $83 \%$ \\
\hline 6 & ${ }^{t} \mathrm{BuOH}$ & $84 \%, 19: 1 \mathrm{dr}$ & $<5 \%$ \\
\hline
\end{tabular}

Table S2: Effect of halogen source and oxidant

$\begin{array}{ccccc}\text { Entry } & \text { "X" (3 equiv) } & \text { Oxidant (3 equiv) } & \text { A } & \text { B } \\ 1 & \mathrm{LiCl} & \mathrm{Phl}(\mathrm{OAc})_{2} & 0 \% & 0 \% \\ 2 & \mathrm{LiBr} & \mathrm{Phl}(\mathrm{OAc})_{2} & 31 \% & <5 \% \\ 3 & \mathrm{Nal} & \mathrm{Phl}(\mathrm{OAc})_{2} & 92 \%, 1.3: 1 \mathrm{dr} & <5 \% \\ 5 & \mathrm{Csl} & \mathrm{Phl}(\mathrm{OAc})_{2} & 90 \%, 1.5: 1 \mathrm{dr} & <5 \% \\ 5 & \mathrm{I}_{2} & \mathrm{Phl}(\mathrm{OAc})_{2} & 84 \%, 19: 1 \mathrm{dr} & <5 \% \\ 6 & \mathrm{I}_{2} & \mathrm{Phl}(\mathrm{OPiv})_{2} & 44 \%, 1.3: 1 \mathrm{dr} & 0 \% \\ 7 & \mathrm{I}_{2} & \mathrm{PlFA} & 17 \% & <5 \% \\ 8 & \mathrm{I}_{2} & \text { none } & 0 \% & 0 \%\end{array}$



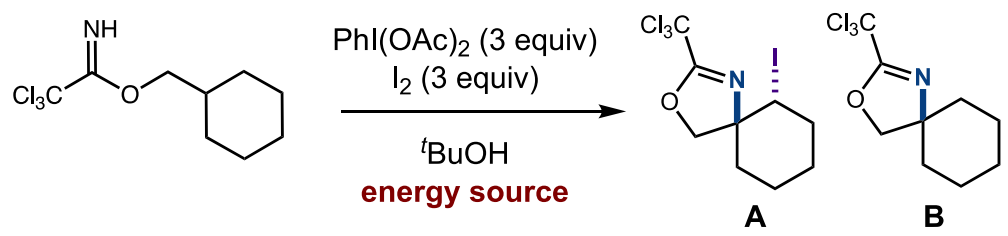

Table S3: Effect of energy source

$\begin{array}{cccc}\text { Entry } & \text { Light } & \text { A } & \text { B } \\ 1 & \text { dark, rt } & 0 & 0 \\ 2 & \text { dark, } 50^{\circ} \mathrm{C} & 20 \% & 0 \\ 3 & \text { white LED } & 84 \%, 2: 1 \mathrm{dr} & <5 \% \\ 4 & \text { white CFL } & 84 \%, 19: 1 \mathrm{dr} & <5 \% \\ 5 & \text { white CFL, no fans } & 86 \%, 9: 1 \mathrm{dr} & <5 \%\end{array}$<smiles>N=C(Cl)OCC1CCCCC1</smiles>
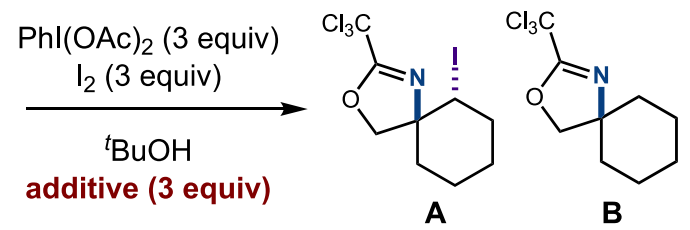

Table S4: Effect of base additives

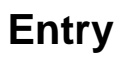

1

2

3

4

5

6
Base Additive

pyridine

collidine

2,6-ditertbutyl pyridine

2,6-Iutidine

2,6-lutidine

none
Solvent

$\mathrm{CH}_{2} \mathrm{Cl}_{2}$

$\mathrm{CH}_{2} \mathrm{Cl}_{2}$

$\mathrm{CH}_{2} \mathrm{Cl}_{2}$

$\mathrm{CH}_{2} \mathrm{Cl}_{2}$

tBuOH

${ }^{t} \mathrm{BuOH}$
A

$47 \%, 4: 1 \mathrm{dr}$ $69 \%, 2: 1 \mathrm{dr}$ $83 \%, 1.3: 1 \mathrm{dr}$ $84 \%, 2: 1 \mathrm{dr}$ $90 \%, 2: 1 \mathrm{dr}$ $84 \%, 19: 1 \mathrm{dr}$

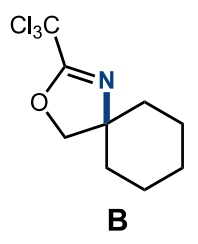

Table S5: Effect of prestir<smiles>N=C(OCC1CCCCC1)C(Cl)(Cl)Cl</smiles>

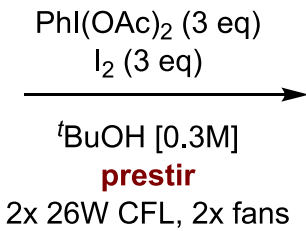<smiles>CC1CCCC2(COC(C(C)(C)C)=N2)C1</smiles>

B $<5 \%$ $<5 \%$ $11 \%$ $5 \%$ $6 \%$ $<5 \%$

$\begin{array}{cccc}\text { Entry } & \text { Pre-stir time } & \text { A } & \text { B } \\ 1 & 0 \mathrm{~min} & 90 \%, 4: 1 \mathrm{dr} & <5 \% \\ 2 & 3 \mathrm{~min} & 84 \%, 19: 1 \mathrm{dr} & <5 \%\end{array}$

Note: with a zero minute prestir, results were inconsistent. 


\section{Functional Group Tolerance and Additive Screen}

Imidate (1) (51.7 mg, $0.2 \mathrm{mmol}$ ) was subjected to GP3 with the following modifications: an additive (1 equiv.) was introduced during the 3 min prestir. Upon completion, dichloroethane or isopropyl acetate was added as an internal standard, and the crude mixture was analyzed by ${ }^{1} \mathrm{H}-\mathrm{NMR}$.<smiles>N=C(OCC1CCCCC1)C(Cl)(Cl)Cl</smiles><smiles>Clc1ccc(Cl)c(Cl)c1</smiles>

Prod: $83 \%(12: 1)$ Additive: $102 \%$<smiles>Clc1ccc2ccccc2n1</smiles>

Prod: $67 \%(20: 1) \quad$ Prod: $78 \%(10: 1)$ Additive: $101 \%$

Additive: $71 \%$

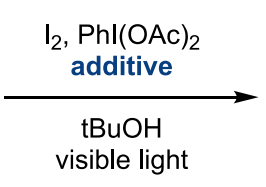

arenes

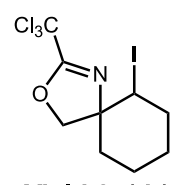

Yield 2, (dr)

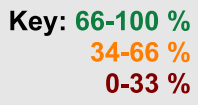

0-33\% carbonyl derivatives<smiles>COC(=O)c1ccccc1</smiles>

Prod: $83 \%(12: 1)$ Additive: $99 \%$<smiles>CC(=O)N(C)c1ccccc1</smiles>

Prod: $76 \%(19: 1)$ Additive: $100 \%$<smiles>O=Cc1ccccc1</smiles>

Prod: $71 \%(12: 1)$ Additive: $84 \%$

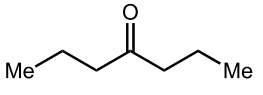

Prod: $75 \%(16: 1)$ Additive: $98 \%$<smiles>[N]C1C=CN=C1</smiles>

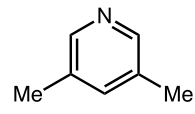

Prod: $71 \%(6: 1)$ Additive: $104 \%$

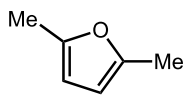

Prod: $20 \%$ (nd) Additive: $0 \%$

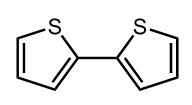

Prod: $17 \%$ (nd) Additive (\%): $0 \%$

other<smiles>CCCCCCl</smiles>

Prod: $78 \%(11: 1)$ Additive: $100 \%$<smiles>CC(C)CO</smiles>

Prod: $71 \%(6: 1)$ Additive: $68 \%$

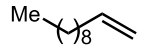

Prod: $92 \%$ (4 : 1)

Additive: $0 \%$

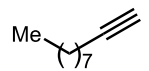

Prod: $89 \%(4: 1)$

Additive: $0 \%$
Prod: $60 \%(11: 1)$ Additive: $73 \%$ 


\section{Amino-iodination Characterization}

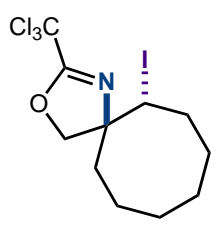

\section{6-iodo-2-(trichloromethyl)-3-oxa-1-azaspiro[4.7]dodec-1-ene (6)}

Imidate S6 (57.3 $\mathrm{mg}, 0.2 \mathrm{mmol}$ ) was subjected to GP3 with the following changes: Nal (90 $\mathrm{mg}, 0.6 \mathrm{mmol})$. Upon completion, the combined organic layer was concentrated in vacuo to afford amino-iodinated product 6 (70\%, $5: 1$ d.r. - NMR yield) as an orange oil with $15 \%$ amination product.

Rf: Diastereomer A (major): 0.32 (5\% EtOAc/hexanes), Diastereomer B (minor): 0.27 (5\% EtOAc/hexanes)

${ }^{1} \mathrm{H}$ NMR (400 MHz, CDCl$)$ : Diastereomer A (major) $\delta=4.61(\mathrm{dd}, \mathrm{J}=9.2 \mathrm{~Hz}, \mathrm{~J}=2.3 \mathrm{~Hz}$, $1 \mathrm{H}), 4.49(\mathrm{ap} \mathrm{s}, 2 \mathrm{H}), 2.82-2.73(\mathrm{~m}, 1 \mathrm{H}), 2.60-2.54(\mathrm{~m}, 1 \mathrm{H}), 2.39-2.32(\mathrm{~m}, 1 \mathrm{H}), 1.89$ $-1.83(\mathrm{~m}, 1 \mathrm{H}), 1.81-1.55(\mathrm{~m}, 7 \mathrm{H}), 1.41-1.34(\mathrm{~m}, 1 \mathrm{H})$.

${ }^{13} \mathrm{C}$ NMR (100 MHz, $\mathbf{C D C l}_{3}$ ): Diastereomer A (major) $\delta=161.62,87.00,83.33,79.14$, $43.46,37.81,33.15,29.44,27.58,24.55,22.16$.

HRMS (ESI-TOF) m/z: calc'd for $\mathrm{C}_{11} \mathrm{H}_{15} \mathrm{Cl}_{3} \mathrm{INO}[\mathrm{M}+\mathrm{Na}]^{+} 431.9156$, found 431.9139 .

IR (neat) cm$^{-1}:$ 2921, 2854, 1663, 1466, 1444, 1269, 998, 789.

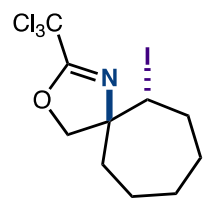

\section{6-iodo-2-(trichloromethyl)-3-oxa-1-azaspiro[4.6]undec-1-ene (7)}

Imidate $\mathbf{S 7}$ ( $54.5 \mathrm{mg}, 0.2 \mathrm{mmol}$ ) was subjected to GP3 with the following changes: Nal (90 $\mathrm{mg}, 0.6 \mathrm{mmol})$. Upon completion, the combined organic layer was concentrated in vacuo to afford amino-iodinated product 7 (82\%, $1.4: 1$ d.r. - NMR yield) as an yellow oil with $10 \%$ amination product.

Rf: Diastereomer A (major): 0.39 (5\% EtOAc/hexanes), Diastereomer B and amination product (minor): 0.45 (5\% EtOAc/hexanes) 
${ }^{1} \mathbf{H}$ NMR (400 MHz, $\left.\mathbf{C D C l}_{3}\right)$ : Diastereomer $\mathbf{A}$ (major) $\delta=4.45(\mathrm{~d}, \mathrm{~J}=8.3 \mathrm{~Hz}, 1 \mathrm{H}), 4.42(\mathrm{~d}$, $\mathrm{J}=8.2 \mathrm{~Hz}, 1 \mathrm{H}), 4.21(\mathrm{dd}, \mathrm{J}=10.3 \mathrm{~Hz}, 2.3 \mathrm{~Hz}, 1 \mathrm{H}), 2.60-2.41(\mathrm{~m}, 2 \mathrm{H}), 2.10-2.04(\mathrm{~m}$, $1 \mathrm{H}), 1.98-1.69(\mathrm{~m}, 4 \mathrm{H}), 1.61-1.34(\mathrm{~m}, 3 \mathrm{H})$.

Diastereomer $\mathbf{B} \delta=4.65(\mathrm{~d}, \mathrm{~J}=8.8 \mathrm{~Hz}, 1 \mathrm{H}), 4.57(\mathrm{dd}, \mathrm{J}=10.0 \mathrm{~Hz}, 2.5 \mathrm{~Hz}, 1 \mathrm{H}), 4.37(\mathrm{~d}$, $\mathrm{J}=8.8 \mathrm{~Hz}, 1 \mathrm{H}), 2.58-2.51(\mathrm{~m}, 1 \mathrm{H}), 2.05-2.02(\mathrm{~m}, 2 \mathrm{H}), 1.99-1.90(\mathrm{~m}, 1 \mathrm{H}), 1.80-$ $1.40(\mathrm{~m}, 6 \mathrm{H})$.

${ }^{13} \mathbf{C}$ NMR (150 MHz, $\mathbf{C D C l}_{3}$ ): Diastereomer $\mathbf{A}$ (major) $\delta=161.26,83.42,78.21,45.22$, 37.28, 36.96, 31.07, 29.02, 27.55, 20.82.

Diastereomer B (minor) $\delta=161.05,80.87,78.39,45.35,37.49,35.98,35.40,27.34$, 26.09, 20.79.

HRMS (ESI-TOF) m/z: calc'd for $\mathrm{C}_{10} \mathrm{H}_{13} \mathrm{Cl}_{3} / \mathrm{NO}[\mathrm{M}+\mathrm{Na}]^{+} 417.9000$, found 417.9023.

IR (neat) cm-1: 2924, 2856, 1661, 1457, 1363, 997, 789.

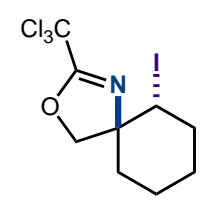

\section{6-iodo-2-(trichloromethyl)-3-oxa-1-azaspiro[4.5]dec-1-ene (2)}

Imidate 1 (51.7 $\mathrm{mg}, 0.2 \mathrm{mmol}$ ) was subjected to GP3. Upon completion, the combined organic layer was concentrated in vacuo before purification by flash chromatography (isocratic: 1 : 99 EtOAc : hexanes) to afford amino-iodinated product 2 (64.3 mg, 84\%, 19 : 1 d.r.) as a white solid.

Note: The product may appear as a yellow oil with the presence of trace amounts of impurities.

Rf: 0.38 (5\% EtOAc/hexanes)

${ }^{1} \mathrm{H}$ NMR (400 MHz, $\left.\mathrm{CDCl}_{3}\right): \delta=4.68(\mathrm{~d}, \mathrm{~J}=8.8 \mathrm{~Hz}, 1 \mathrm{H}), 4.53(\mathrm{dd}, \mathrm{J}=11.7 \mathrm{~Hz}, \mathrm{~J}=4.0$ $\mathrm{Hz}, 1 \mathrm{H}), 4.42(\mathrm{~d}, \mathrm{~J}=8.8 \mathrm{~Hz}, 1 \mathrm{H}), 2.51-2.44(\mathrm{~m}, 1 \mathrm{H}), 2.12-2.06(\mathrm{~m}, 1 \mathrm{H}), 1.98-1.80$ $(\mathrm{m}, 3 \mathrm{H}), 1.68-1.55(\mathrm{~m}, 1 \mathrm{H}), 1.51-1.42(\mathrm{~m}, 1 \mathrm{H}), 1.37-1.25(\mathrm{~m}, 1 \mathrm{H})$.

${ }^{13} \mathrm{C}$ NMR $\left(150 \mathrm{MHz}, \mathrm{CDCl}_{3}\right): \delta=161.64,86.76,79.51,75.50,41.08,36.18,36.14,26.99$, 21.96.

HRMS (ESI-TOF) m/z: calc'd for $\mathrm{C}_{9} \mathrm{H}_{11} \mathrm{Cl}_{3} \mathrm{INO}[\mathrm{M}+\mathrm{Na}]^{+} 403.8843$, found 403.8856 .

IR (neat) cm$^{-1}:$ 2936, 2858, 1657, 1446, 1301, 1240, 999, 777.

MP: $58.0^{\circ} \mathrm{C}-60.6^{\circ} \mathrm{C}$. 


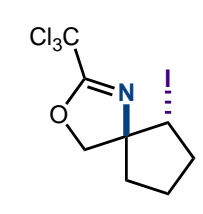

\section{6-iodo-2-(trichloromethyl)-3-oxa-1-azaspiro[4.4]non-1-ene (8)}

Imidate S8 (48.9 mg, $0.2 \mathrm{mmol}$ ) was subjected to GP3 with the following changes: Nal $(90 \mathrm{mg}, 0.6 \mathrm{mmol})$. Upon completion, the combined organic layer was concentrated in vacuo before purification by flash chromatography (isocratic: $1: 99 \mathrm{EtOAc}:$ hexanes) to afford amino-iodinated product 8 (72.6 $\mathrm{mg}, 99 \%, 1: 1$ d.r.) as a yellow oil for one diastereomer $(\mathbf{A})$ and a white solid for the other diastereomer (B).

Rf: Diastereomer A: $0.34 \quad(5 \%$ EtOAc/hexanes $)$, Diastereomer $\quad$ B: $0.24 \quad(5 \%$ $\mathrm{EtOAc/hexanes)}$

${ }^{1} \mathrm{H}$ NMR (400 MHz, $\left.\mathbf{C D C l}_{3}\right)$ : Diastereomer $\mathbf{A} \delta=4.81(\mathrm{~d}, \mathrm{~J}=9.2 \mathrm{~Hz}, 1 \mathrm{H}), 4.40(\mathrm{~d}, \mathrm{~J}=9.2$ $\mathrm{Hz}, 1 \mathrm{H}), 4.34(\mathrm{ap} \mathrm{t}, \mathrm{J}=6.3 \mathrm{~Hz}, 1 \mathrm{H}), 2.62-2.54(\mathrm{~m}, 1 \mathrm{H}), 2.18-2.02(\mathrm{~m}, 3 \mathrm{H}), 1.96-$ $1.88(\mathrm{~m}, 2 \mathrm{H})$.

Diastereomer B $\delta=4.48(\mathrm{~d}, \mathrm{~J}=8.8 \mathrm{~Hz}, 1 \mathrm{H}), 4.44(\mathrm{~d}, \mathrm{~J}=8.9 \mathrm{~Hz}, 1 \mathrm{H}), 3.96(\mathrm{dd}, \mathrm{J}=11.0$ $\mathrm{Hz}, \mathrm{J}=7.7 \mathrm{~Hz}, 1 \mathrm{H}), 2.47-2.31(\mathrm{~m}, 2 \mathrm{H}), 2.18-2.10(\mathrm{~m}, 1 \mathrm{H}), 2.05-1.97(\mathrm{~m}, 1 \mathrm{H}), 1.86$ $-1.72(\mathrm{~m}, 2 \mathrm{H})$.

${ }^{13} \mathrm{C}$ NMR (100 MHz, $\left.\mathbf{C D C l}_{3}\right)$ : Diastereomer $\mathbf{A} \delta=162.51,86.77,82.08,81.66,38.08$, $36.48,35.68,22.08$.

Diastereomer B $\delta=161.91,86.82,80.42,77.40,36.27,35.97,34.96,23.11$.

HRMS (ESI-TOF) m/z: calc'd for $\mathrm{C}_{8} \mathrm{H}_{9} \mathrm{Cl}_{3} \mathrm{INO}[\mathrm{M}+\mathrm{H}]^{+} 367.8867$, found 367.8864 .

IR (neat) cm$^{-1}:$ 2982, 2958, 1654, 1466, 1444, 1271, 996, 788, 773.

MP (Diastereomer B): $113.0^{\circ} \mathrm{C}-114.8^{\circ} \mathrm{C}$.

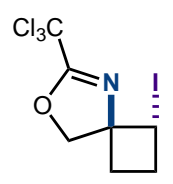

\section{1-iodo-6-(trichloromethyl)-7-oxa-5-azaspiro[3.4]oct-5-ene (9)}

Imidate S9 (46.1 $\mathrm{mg}, 0.2 \mathrm{mmol}$ ) was subjected to GP3 with the following changes: $\mathrm{Phl}(\mathrm{OAc})_{2}(257.3 \mathrm{mg}, 0.8 \mathrm{mmol})$ and $\mathrm{I}_{2}(101.5 \mathrm{mg}, 0.4 \mathrm{mmol})$. Upon completion, the combined organic layer was concentrated in vacuo before purification by flash chromatography (isocratic: $1: 99 \mathrm{EtOAc}:$ hexanes) to afford amino-iodinated product 9 (64.4 mg, 91\%, $1: 1 \mathrm{~d}$. r.) as a white solid for diastereomer $\mathbf{A}$ (major) and a yellow oil for diastereomer B (minor). 
Rf: Diastereomer A: $0.25 \quad(5 \% \quad$ EtOAc/hexanes $)$, Diastereomer $\quad$ B: $0.20 \quad(5 \%$ EtOAc/hexanes)

${ }^{1} \mathrm{H}$ NMR (400 MHz, $\left.\mathbf{C D C l}_{3}\right)$ : Diastereomer $\mathbf{A} \delta=5.13(\mathrm{~d}, \mathrm{~J}=9.4 \mathrm{~Hz}, 1 \mathrm{H}), 4.87(\mathrm{dd}, \mathrm{J}=$ $9.0 \mathrm{~Hz}, 9.0 \mathrm{~Hz}, 1 \mathrm{H}), 4.58(\mathrm{~d}, \mathrm{~J}=9.3 \mathrm{~Hz}, 1 \mathrm{H}), 2.78-2.70(\mathrm{~m}, 1 \mathrm{H}), 2.65-2.57(\mathrm{~m}, 1 \mathrm{H})$, $2.39-2.33(\mathrm{~m}, 1 \mathrm{H}), 2.16-2.04(\mathrm{~m}, 1 \mathrm{H})$.

Diastereomer $\mathbf{B} \delta=4.45(\mathrm{dd}, \mathrm{J}=10.0 \mathrm{~Hz}, 8.0 \mathrm{~Hz}, 1 \mathrm{H}), 4.36(\mathrm{~d}, \mathrm{~J}=9.8 \mathrm{~Hz}, 1 \mathrm{H}), 4.32$ $(\mathrm{d}, \mathrm{J}=9.6 \mathrm{~Hz}, 1 \mathrm{H}), 2.80-2.70(\mathrm{~m}, 1 \mathrm{H}), 2.55-2.46(\mathrm{~m}, 2 \mathrm{H}), 2.38-2.30(\mathrm{~m}, 1 \mathrm{H})$.

${ }^{13} \mathrm{C}$ NMR (100 MHz, $\left.\mathbf{C D C l}_{3}\right)$ : Diastereomer A $\delta=163.45,86.41,83.14,76.13,34.04$, 28.93, 27.42.

Diastereomer B $\delta=163.12,86.53,78.34,77.06,34.29,29.04,25.60$.

HRMS (ESI-TOF) m/z: calc'd for $\mathrm{C}_{7} \mathrm{H}_{7} \mathrm{Cl}_{3} \mathrm{INO}[\mathrm{M}+\mathrm{H}]^{+}$353.8711, found 353.8708.

IR (film) $\mathbf{c m}^{-1}:$ 2923, 2853, 1711, 1654, 1506, 1461, 819, 792.

MP (Diastereomer A): $114.4^{\circ} \mathrm{C}-117.0^{\circ} \mathrm{C}$.

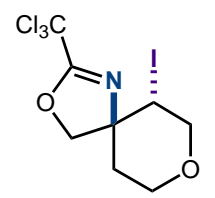

6-iodo-2-(trichloromethyl)-3,8-dioxa-1-azaspiro[4.5]dec-1-ene (10)

Imidate S10 (52.1 mg, $0.2 \mathrm{mmol}$ ) was subjected to GP3 with the following changes: the reaction time was extended to $2 \mathrm{~h}$. Upon completion, the combined organic layer was concentrated in vacuo before purification by flash chromatography (isocratic: $5: 94$ EtOAc : hexanes) to afford amino-iodinated product $10(53.9 \mathrm{mg}, 70 \%, 1.6: 1 \mathrm{~d}$.r. $)$ as a yellow oil with $5 \%$ amination product.

Rf: Diastereomer A \& B: 0.32 (15\% EtOAc/hexanes)

${ }^{1} \mathrm{H}$ NMR (400 MHz, $\left.\mathrm{CDCl}_{3}\right)$ : Diastereomer A \& B $\delta=4.73(\mathrm{~d}, \mathrm{~J}=8.9 \mathrm{~Hz}, 1 \mathrm{H}), 4.50-$ $4.44(\mathrm{~m}, 3.5 \mathrm{H}), 4.25-4.17(\mathrm{~m}, 4 \mathrm{H}), 4.12-4.02(\mathrm{~m}, 5.6 \mathrm{H}), 3.96-3.92(\mathrm{~m}, 1.4 \mathrm{H})$, $3.55-3.46(\mathrm{~m}, 2 \mathrm{H}), 2.33-2.10(\mathrm{~m}, 5.6 \mathrm{H})$.

${ }^{13} \mathrm{C}$ NMR (100 MHz, $\mathbf{C D C l}_{3}$ ): Diastereomer A \& B $\delta=162.76,162.28,86.78,86.58$, 81.51, 79.66, 73.30, 72.28, 71.11, 71.05, 64.66, 64.39, 39.05, 38.28, 37.52, 37.05, $36.70,29.83$.

HRMS (ESI-TOF) m/z: calc'd for $\mathrm{C}_{8} \mathrm{H}_{9} \mathrm{Cl}_{3} \mathrm{INO}_{2}[\mathrm{M}+\mathrm{Na}]^{+} 405.8636$, found 405.8645 .

IR (neat) cm$^{-1}:$ 2962, 2847, 1735, 1659, 1465, 1436, 1243, 1095, 1007, 785. 


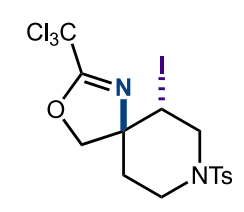

\section{6-iodo-8-tosyl-2-(trichloromethyl)-3-oxa-1,8-diazaspiro[4.5]dec-1-ene (11)}

Imidate (S11) (82.7 mg, $0.2 \mathrm{mmol}$ ) was subjected to GP3. Upon completion, the combined organic layer was concentrated and the residue was loaded directly onto silica gel and purified by flash column chromatography (isocratic: $10: 90$, EtOAc : hexanes) to yield amino-iodinated product 11 (79.6 $\mathrm{mg}, 74 \%, 14: 1)$ as a pale yellow solid.

Rf: 0.40 (20\% EtOAc / hexanes).

${ }^{1} \mathrm{H}$ NMR (400 MHz, $\left.\mathrm{CDCl}_{3}\right): \delta=7.66(\mathrm{~d}, \mathrm{~J}=8.2 \mathrm{~Hz}, 2 \mathrm{H}), 7.35(\mathrm{~d}, \mathrm{~J}=8.0 \mathrm{~Hz}, 2 \mathrm{H}), 4.49$ $(\mathrm{d}, \mathrm{J}=9.0 \mathrm{~Hz}, 1 \mathrm{H}), 4.39(\mathrm{dd}, \mathrm{J}=8.7,3.7 \mathrm{~Hz}, 1 \mathrm{H}), 4.25(\mathrm{~d}, \mathrm{~J}=9.3 \mathrm{~Hz}, 1 \mathrm{H}), 3.88-3.85(\mathrm{~m}$, $1 \mathrm{H})$, 3.57-3.54 (m, $1 \mathrm{H}), 3.05-3.00(\mathrm{~m}, 1 \mathrm{H}), 2.86-2.82(\mathrm{~m}, 1 \mathrm{H}), 2.45(\mathrm{~s}, 3 \mathrm{H}), 2.28-2.22(\mathrm{~m}$, $1 \mathrm{H}), 2.15-2.06(\mathrm{~m}, 1 \mathrm{H})$.

${ }^{13} \mathrm{C}$ NMR $\left(100 \mathrm{MHz}, \mathrm{CDCl}_{3}\right): \delta=162.77,144.24,133.91,130.13,127.64,86.44,79.97$, $73.25,51.14,42.52,35.01,34.00,21.73$.

HRMS (ESI-TOF) m/z: calc'd for $\mathrm{C}_{15} \mathrm{H}_{16} \mathrm{Cl}_{3} \mathrm{IN}_{2} \mathrm{O}_{3} \mathrm{~S}$ [M+Na] ${ }^{+} 558.8890$, found 558.8890 .

IR (film) cm-1: 3065, 3048, 2971, 2925, 2859, 2361, 1723, 1667, 1352, 1152, 786.

MP: $208.1-209.0^{\circ} \mathrm{C}$.

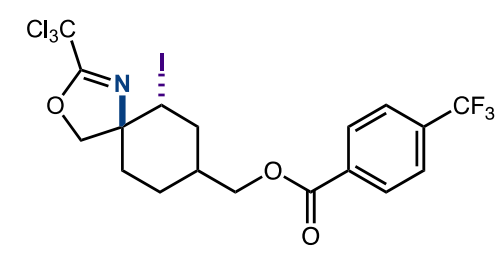

\section{6-iodo-2-(trichloromethyl)-3-oxa-1-azaspiro[4.5]dec-1-en-8-yl)methyl 4- (trifluoromethyl)benzoate (12)}

Imidate S12 (92.1 mg, $0.2 \mathrm{mmol})$ was subjected to GP3. Upon completion, the combined organic layer was concentrated in vacuo before purification by flash chromatography (isocratic: 10 : 90, EtOAc : hexanes) to afford amino-iodinated product 12 (95.9 mg, 82\%, 13:1) as a white solid.

Rf: 0.58 (15\% EtOAc/hexanes) 
${ }^{1} \mathrm{H}$ NMR $\left(600 \mathrm{MHz}, \mathrm{CDCl}_{3}\right): \delta=8.16(\mathrm{~d}, \mathrm{~J}=8.1 \mathrm{~Hz}, 2 \mathrm{H}), 7.71(\mathrm{~d}, \mathrm{~J}=8.3 \mathrm{~Hz}, 2 \mathrm{H}), 4.50-$ $4.49(\mathrm{~m}, 1 \mathrm{H}), 4.46(\mathrm{~d}, \mathrm{~J}=9.1 \mathrm{~Hz}, 1 \mathrm{H}), 4.35-4.32(\mathrm{~m}, 2 \mathrm{H}), 4.26(\mathrm{~d}, \mathrm{~J}=9.2 \mathrm{~Hz}, 1 \mathrm{H}), 2.48-$ $2.41(\mathrm{~m}, 1 \mathrm{H}), 2.37-2.32(\mathrm{~m}, 1 \mathrm{H}), 2.29-2.24(\mathrm{~m}, 1 \mathrm{H}), 2.13-2.10(\mathrm{~m}, 1 \mathrm{H}), 1.85-1.81(\mathrm{~m}, 2 \mathrm{H})$, $1.73-1.71(\mathrm{~m}, 1 \mathrm{H})$.

${ }^{13} \mathrm{C}$ NMR (150 MHz, $\left.\mathrm{CDCl}_{3}\right): \delta=165.36,161.78,134.68(\mathrm{q}, 32.6 \mathrm{~Hz}), 133.53,130.15$, 125.60 (q, 3.7 Hz), 123.75 (q, $272.5 \mathrm{~Hz}$ ), 87.05, 83.46, 74.34, 68.60, 39.12, 35.76, 33.25, $33.25,33.00,24.31$.

${ }^{19} \mathrm{~F}$ NMR (564 MHz, $\left.\mathrm{CDCl}_{3}\right): \delta=-63.12$.

HRMS (ESI-TOF) m/z: calc'd for $\mathrm{C}_{18} \mathrm{H}_{16} \mathrm{Cl}_{3} \mathrm{~F}_{3} \mathrm{INO}_{3}[\mathrm{M}+\mathrm{H}]^{+} 460.0461$, found 460.0462 .

IR (film) cm': 3058, 2946, 1721, 1658, 1271, 774.

MP: $126.0-128.0^{\circ} \mathrm{C}$.

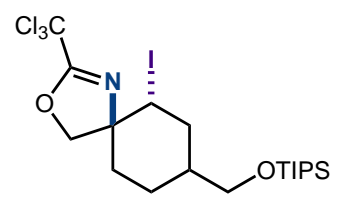

6-iodo-2-(trichloromethyl)-8-(((triisopropylsilyl)oxy)methyl)-3-oxa-1azaspiro[4.5]dec-1-ene (13)

Imidate (S13) (89 $\mathrm{mg}, 0.2 \mathrm{mmol}$ ) was subjected to GP3. Upon completion, the combined organic layer was concentrated and the residue was loaded directly onto silica gel and purified by flash column chromatography (isocratic: $5: 94: 1$, EtOAc : hexanes : Et ${ }_{3} \mathrm{~N}$ ) to yield imidate $\mathrm{X}(89.9 \mathrm{mg}, 79 \%)$ as a colorless oil.

Rf: 0.46 (10\% EtOAc / hexanes).

${ }^{1} \mathrm{H}$ NMR (400 MHz, CDCl$): ~ \delta=4.53-4.50(\mathrm{~m}, 1 \mathrm{H}), 4.49(\mathrm{~d}, \mathrm{~J}=9.0 \mathrm{~Hz}, 1 \mathrm{H}), 4.28(\mathrm{~d}, \mathrm{~J}=$ $4.5 \mathrm{~Hz}, 1 \mathrm{H}), 3.66(\mathrm{dd}, \mathrm{J}=6.0,2.0 \mathrm{~Hz}, 2 \mathrm{H}), 2.30-2.23(\mathrm{~m}, 1 \mathrm{H}), 2.19-2.11(\mathrm{~m}, 1 \mathrm{H}), 2.09-$ $2.02(\mathrm{~m}, 2 \mathrm{H}), 1.84-1.74(\mathrm{~m}, 2 \mathrm{H}), 1.61-1.57(\mathrm{~m}, 1 \mathrm{H}), 1.07-1.06(\mathrm{~m}, 2 \mathrm{H})$.

${ }^{13} \mathrm{C}$ NMR (150 MHz, $\left.\mathrm{CDCl}_{3}\right): \delta=161.50,87.10,83.01,74.84,66.48,40.44,36.72,36.11$, $33.17,28.85,23.92,18.18,12.11$.

HRMS (ESI-TOF) m/z: calc'd for $\mathrm{C}_{19} \mathrm{H}_{33} \mathrm{Cl}_{3} \mathrm{INO}_{2} \mathrm{Si}[\mathrm{M}+\mathrm{Na}]^{+} 590.0289$, found 590.0272 .

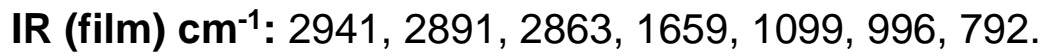




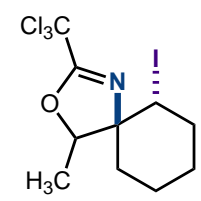

\section{6-iodo-4-methyl-2-(trichloromethyl)-3-oxa-1-azaspiro[4.5]dec-1-ene (14)}

Imidate $\mathbf{S 1 4}$ (54.5 mg, $0.2 \mathrm{mmol}$ ) was subjected to GP3 with the following changes: Nal $(90 \mathrm{mg}, 0.6 \mathrm{mmol})$. Upon completion, the combined organic layer was concentrated in vacuo before purification by flash chromatography (isocratic: $1: 99$ EtOAc : hexanes) to afford amino-iodinated product 14 (42.6 mg, 54\%, $1.3: 1$ d.r.) as an orange oil for both diastereomers with $5 \%$ amination product.

Rf: Diastereomer A (major): 0.34 (5\% EtOAc/hexanes), Diastereomer B (minor): 0.29 (5\% EtOAc/hexanes)

${ }^{1} \mathrm{H}$ NMR (400 MHz, $\mathrm{CDCl}_{3}$ ): Diastereomer A (major) $\delta=4.78(\mathrm{q}, \mathrm{J}=6.6 \mathrm{~Hz}, 1 \mathrm{H}), 4.41-$ $4.39(\mathrm{~m}, 1 \mathrm{H}), 2.45-2.37(\mathrm{~m}, 1 \mathrm{H}), 2.03-1.50(\mathrm{~m}, 7 \mathrm{H}), 1.40(\mathrm{~d}, \mathrm{~J}=6.5 \mathrm{~Hz}, 3 \mathrm{H})$.

Diastereomer B (minor) $\delta=4.91(\mathrm{q}, \mathrm{J}=6.6 \mathrm{~Hz}, 1 \mathrm{H}), 4.20(\mathrm{dd}, \mathrm{J}=12.1 \mathrm{~Hz}, \mathrm{~J}=4.2 \mathrm{~Hz}$, $1 \mathrm{H}), 2.67-2.57(\mathrm{~m}, 1 \mathrm{H}), 2.43-2.37(\mathrm{~m}, 1 \mathrm{H}), 2.01-1.84(\mathrm{~m}, 2 \mathrm{H}), 1.72-1.64(\mathrm{~m}, 2 \mathrm{H})$, $1.58-1.51(\mathrm{~m}, 1 \mathrm{H}), 1.44-1.33(\mathrm{~m}, 1 \mathrm{H}), 1.36(\mathrm{~d}, \mathrm{~J}=6.7 \mathrm{~Hz}, 3 \mathrm{H})$.

${ }^{13} \mathbf{C}$ NMR (100 MHz, $\mathbf{C D C l}_{3}$ ): Diastereomer A (major) $\delta=160.44,89.63,87.29,74.72$, 44.60, 33.92, 29.38, 23.80, 22.06, 16.13.

Diastereomer B (minor) $\delta=161.00,89.87,87.40,74.92,44.98,37.59,32.48,29.10$, $21.75,15.47$.

HRMS (ESI-TOF) m/z: calc'd for $\mathrm{C}_{10} \mathrm{H}_{13} \mathrm{Cl}_{3} \mathrm{INO}[\mathrm{M}+\mathrm{H}]^{+} 395.9180$, found 395.9182 .

IR (neat) $\mathbf{c m}^{-1}:$ 2964, 2858, 1700, 1656, 1445, 1380, 1249, 824, 789.

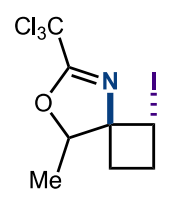

\section{1-iodo-8-methyl-6-(trichloromethyl)-7-oxa-5-azaspiro[3.4]oct-5-ene (15)}

Imidate $\mathbf{S} 15$ (48.9 mg, $0.2 \mathrm{mmol}$ ) was subjected to GP3 with the following changes: Nal $(90 \mathrm{mg}, 0.6 \mathrm{mmol})$. Upon completion, the combined organic layer was concentrated in vacuo before purification by flash chromatography (isocratic: $1: 99 \mathrm{EtOAc}$ : hexanes) to afford amino-iodinated product $15(42.6 \mathrm{mg}, 54 \%, 3.3: 3.2: 1 \mathrm{~d} . \mathrm{r}$.) as an orange oil for both diastereomers with $5 \%$ of another diastereomer. 
Rf: Diastereomer A (major): 0.28 (5\% EtOAc/hexanes), Diastereomer B (minor): 0.18 (5\% EtOAc/hexanes)

${ }^{1} \mathrm{H}$ NMR (400 MHz, $\mathbf{C D C l}_{3}$ ): Diastereomer $\mathbf{A}$ (major) $\delta=5.21(\mathrm{q}, \mathrm{J}=6.6 \mathrm{~Hz}, 1 \mathrm{H}), 4.21$ (ap $\mathrm{t}, \mathrm{J}=8.3 \mathrm{~Hz}, 1 \mathrm{H}), 2.76-2.68(\mathrm{~m}, 1 \mathrm{H}), 2.55-2.34(\mathrm{~m}, 3 \mathrm{H}), 1.49(\mathrm{~d}, \mathrm{~J}=6.5 \mathrm{~Hz}, 3 \mathrm{H})$. Diastereomer B (minor) $\delta=4.57(\mathrm{q}, \mathrm{J}=6.7 \mathrm{~Hz}, 1 \mathrm{H}), 4.37$ (dd, $\mathrm{J}=10.0 \mathrm{~Hz}, \mathrm{~J}=8.0 \mathrm{~Hz}$, $1 \mathrm{H}), 2.80-2.70(\mathrm{~m}, 1 \mathrm{H}), 2.51-2.26(\mathrm{~m}, 3 \mathrm{H}), 1.36(\mathrm{~d}, \mathrm{~J}=6.5 \mathrm{~Hz}, 3 \mathrm{H})$.

${ }^{13} \mathbf{C}$ NMR (150 MHz, $\mathbf{C D C l}_{3}$ ): Diastereomer A (major) $\delta=162.43,89.08,86.73,77.90$, 29.72, 29.40, 28.94, 16.95 .

Diastereomer B (minor) $\delta=162.42,86.87,85.35,79.77,29.95,29.25,26.16,16.19$.

HRMS (ESI-TOF) m/z: calc'd for $\mathrm{C}_{8} \mathrm{H}_{9} \mathrm{Cl}_{3} \mathrm{INO}[\mathrm{M}+\mathrm{H}]^{+} 367.8867$, found 367.8840 .

IR (film) cm': 2969, 2932, 1710, 1466, 1378, 1340, 1305.

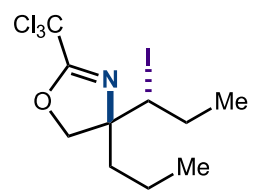

\section{4-((1-iodopropyl)-4-propyl-2-(trichloromethyl)-4,5-dihydrooxazole (16)}

Imidate (16) $(74.9 \mathrm{mg}, 0.2 \mathrm{mmol})$ was subjected to GP3. Upon completion, the combined organic layer was concentrated and the residue was loaded directly onto silica gel and purified by flash column chromatography (isocratic: hexanes) to yield amino-iodinated product $16(66.9 \mathrm{mg}, 82 \%, 1.1: 1)$ as a pale yellow oil.

Rf: 0.53 (10\% EtOAc / hexanes).

${ }^{1} \mathrm{H}$ NMR (400 MHz, $\left.\mathbf{C D C l}_{3}\right)$ : $\delta$ = Diastereomer A: $4.64(\mathrm{~d}, \mathrm{~J}=9.3 \mathrm{~Hz}, 1 \mathrm{H}), 4.34(\mathrm{~d}, \mathrm{~J}=9.2$ $\mathrm{Hz}, 1 \mathrm{H}), 4.20-4.16(\mathrm{~m}, 1 \mathrm{H}), 2.03-1.30(\mathrm{~m}, 6 \mathrm{H}), 0.96(\mathrm{t}, \mathrm{J}=7.3 \mathrm{~Hz}, 6 \mathrm{H})$.

Diastereomer B: $4.50(\mathrm{~d}, \mathrm{~J}=9.0 \mathrm{~Hz}, 1 \mathrm{H}), 4.44(\mathrm{~d}, \mathrm{~J}=9.0 \mathrm{~Hz}, 1 \mathrm{H}), 4.20-4.16(\mathrm{~m}, 1 \mathrm{H})$, 2.03-1.30 (m, 6H), $1.08(\mathrm{t}, \mathrm{J}=7.1 \mathrm{~Hz}, 6 \mathrm{H})$.

${ }^{13} \mathrm{C}$ NMR (150 MHz, $\left.\mathrm{CDCl}_{3}\right): \delta=161.94,161.74,86.86,79.94,78.21,77.87,77.82,51.34$, 47.63, 43.93, 38.21, 27.72, 27.31, 17.13, 16.66, 15.20, 15.07, 14.43, 14.29.

HRMS (ESI-TOF) m/z: calc'd for $\mathrm{C}_{10} \mathrm{H}_{15} \mathrm{Cl}_{3} \mathrm{INO}[\mathrm{M}+\mathrm{Na}]^{+} 419.9162$, found 419.9140 .

IR (film) cm': 2964, 2937, 2874, 1770, 1659, 998, 816, 750. 


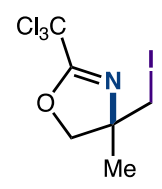

\section{4-(iodomethyl)-4-methyl-2-(trichloromethyl)-4,5-dihydrooxazole (17)}

Imidate $\mathbf{S 1 7}$ (43.7 $\mathrm{mg}, 0.2 \mathrm{mmol}$ ) was subjected to GP3 with the following changes: $\mathrm{Phl}(\mathrm{OAc})_{2}(322.1 \mathrm{mg}, 1.0 \mathrm{mmol})$ and $\mathrm{Nal}(90 \mathrm{mg}, 0.6 \mathrm{mmol})$. In addition, the reaction time was extended to $2 \mathrm{~h}$. Upon completion, the combined organic layer was concentrated in vacuo before purification by flash chromatography (isocratic: $5: 94$ EtOAc : hexanes) to afford amino-iodinated product $17(34.4 \mathrm{mg}, 50 \%)$ as a yellow solid.

Rf: 0.22 (5\% EtOAc/hexanes)

${ }^{1} \mathrm{H}$ NMR $\left(400 \mathrm{MHz}, \mathrm{CDCl}_{3}\right): \delta=4.58(\mathrm{~d}, \mathrm{~J}=8.4 \mathrm{~Hz}, 1 \mathrm{H}), 4.30(\mathrm{~d}, \mathrm{~J}=8.8 \mathrm{~Hz}, 1 \mathrm{H}), 3.39$ (ap q, J = 8.1 Hz, 2H), $1.60(\mathrm{~s}, 3 \mathrm{H})$.

${ }^{13} \mathrm{C}$ NMR $\left(100 \mathrm{MHz}, \mathrm{CDCl}_{3}\right): \delta=162.68,86.61,81.27,71.11,26.04,15.46$.

HRMS (ESI-TOF) m/z: calc'd for $\mathrm{C}_{6} \mathrm{H}_{7} \mathrm{Cl}_{3} \mathrm{INO}[\mathrm{M}+\mathrm{H}]^{+} 341.8711$, found 341.8701 .

IR (neat) $\mathbf{c m}^{-1}: 2975,1652,1471,1290,788,776$.

MP: $59.0^{\circ} \mathrm{C}-62.7^{\circ} \mathrm{C}$.
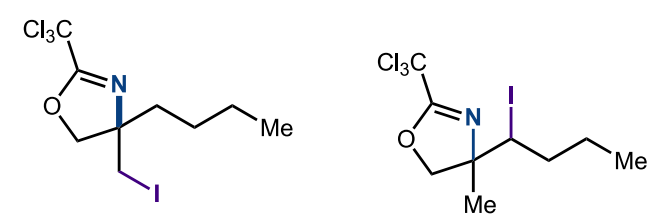

\section{4-(1-iodobutyl)-4-methyl-2-(trichloromethyl)-4,5-dihydrooxazole (18)}

Imidate (18) (52.1 $\mathrm{mg}, 0.2 \mathrm{mmol}$ ) was subjected to GP3. Upon completion, the combined organic layer was concentrated and the residue was loaded directly onto silica gel and purified by flash column chromatography (isocratic: $1: 99$ EtOAc : hexanes) to yield amino-iodinated product $18(56.9 \mathrm{mg}, 74 \%, 1.1: 1 \mathrm{dr}, 8: 1 \mathrm{rr})$ as a pale yellow oil.

Rf: 0.36 (10\% EtOAc / hexanes).

${ }^{1} \mathrm{H}$ NMR $\left(400 \mathrm{MHz}, \mathrm{CDCl}_{3}\right): \delta$ = Diastereomer A: $4.70(\mathrm{~d}, \mathrm{~J}=9.1 \mathrm{~Hz}, 1 \mathrm{H}), 4.28(\mathrm{~d}, \mathrm{~J}=9.1$ $\mathrm{Hz}, 1 \mathrm{H}), 4.21$ (dd, J = 11.3 Hz, 2.9 Hz, 1H), 1.94-1.28 (m, 6H), 0.98-0.92 (m, 3H).

Diastereomer B: $4.51(\mathrm{~d}, \mathrm{~J}=8.8 \mathrm{~Hz}, 1 \mathrm{H}), 4.33(\mathrm{~d}, \mathrm{~J}=8.8 \mathrm{~Hz}, 1 \mathrm{H}), 4.14(\mathrm{dd}, \mathrm{J}=11.3 \mathrm{~Hz}$, $2.5 \mathrm{~Hz}, 1 \mathrm{H}), 1.94-1.28(\mathrm{~m}, 6 \mathrm{H}), 0.98-0.92(\mathrm{~m}, 3 \mathrm{H})$. 
Regioisomer 2: $4.51(\mathrm{~d}, \mathrm{~J}=8.9 \mathrm{~Hz}, 1 \mathrm{H}), 4.39(\mathrm{~d}, \mathrm{~J}=8.9 \mathrm{~Hz}, 1 \mathrm{H}), 3.40(\mathrm{dd}, \mathrm{J}=17.6,10.4$ $\mathrm{Hz}, 2 \mathrm{H}), 1.94-1.28(\mathrm{~m}, 6 \mathrm{H}), 0.98-0.92(\mathrm{~m}, 3 \mathrm{H})$.

${ }^{13} \mathrm{C}$ NMR (150 MHz, $\mathrm{CDCl}_{3}$ ): $\delta=161.97,161.95,161.89,86.67,83.03,80.03,76.48$, 75.36, 74.90, 74.42, 73.68, 71.32, 47.19, 45.32, 39.41, 36.50, 36.11, 31.59, 29.34, 25.95, $23.59,23.51,23.33,23.28,23.07,14.22,13.23,13.20$.

HRMS (ESI-TOF) m/z: calc'd for $\mathrm{C}_{9} \mathrm{H}_{13} \mathrm{Cl}_{3} \mathrm{INO}[\mathrm{M}+\mathrm{Na}]^{+} 405.9005$, found 405.8994 .

IR (film) cm$^{-1}:$ 2926, 2855, 2359, 1660, 999, 795.

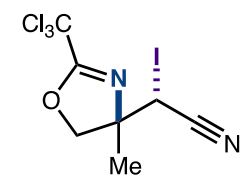

2-(4-(iodomethyl)-2-(trichloromethyl)-4,5-dihydrooxazol-4-yl)acetonitrile (19)

Imidate S19 (48.7 mg, $0.2 \mathrm{mmol})$ was subjected to GP3 with the following changes: $\mathrm{Phl}(\mathrm{OAc}) 2$ (257.3 $\mathrm{mg}, 0.8 \mathrm{mmol})$. Upon completion, the combined organic layer was concentrated and the yield was determined by ${ }^{1} \mathrm{H}-\mathrm{NMR}$ using dichloroethane as an internal standard $(75 \%, 1: 1)$. Compound 19 was isolated using preparatory thin-layer chromatography (10:90 EtOAc : hexanes) as a yellow oil

Rf: Diastereomer A: 0.21 (10\% EtOAc/hexanes), Diastereomer B: $0.19 \quad(10 \%$ EtOAc/hexanes)

${ }^{1} \mathrm{H}$ NMR (400 MHz, $\left.\mathrm{CDCl}_{3}\right)$ : Diastereomer A $\delta=4.75(\mathrm{~d}, \mathrm{~J}=9.5 \mathrm{~Hz}, 1 \mathrm{H}), 4.54(\mathrm{~s}, 1 \mathrm{H})$, $4.46(\mathrm{~d}, \mathrm{~J}=9.4 \mathrm{~Hz}, 1 \mathrm{H}), 1.80(\mathrm{~s}, 3 \mathrm{H})$.

Diastereomer B $\delta=4.76(d, J=9.5 \mathrm{~Hz}, 1 \mathrm{H}), 4.44(\mathrm{~s}, 1 \mathrm{H}), 4.41(\mathrm{~d}, \mathrm{~J}=9.5 \mathrm{~Hz}, 1 \mathrm{H}), 1.68$ (s, $3 \mathrm{H})$.

${ }^{13} \mathrm{C}$ NMR (100 MHz, $\mathbf{C D C l}_{3}$ ): Diastereomer A $\delta=164.00,116.80,86.07,80.02,73.11$, 24.14, 8.97.

Diastereomer B $\delta=164.46,116.44,80.01,79.45,73.57,26.34,7.09$.

HRMS (ESI-TOF) m/z: calc'd for $\mathrm{C}_{7} \mathrm{H}_{6} \mathrm{Cl}_{3} \mathrm{IN}_{2} \mathrm{O}[\mathrm{M}+\mathrm{Na}]^{+} 388.8483$, found 388.8465 .

IR (neat) $\mathbf{c m}^{-1}:$ 2975, 1652, 1471, 1290, 788, 776. 


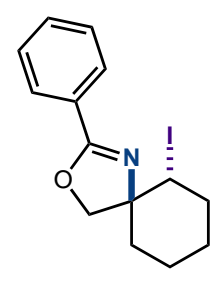

6-iodo-2-phenyl-3-oxa-1-azaspiro[4.5]dec-1-ene (20)

Imidate (S20) (43.5 mg, $0.2 \mathrm{mmol}$ ) was subjected to GP3. Upon completion, the combined organic layer was concentrated and the residue was loaded directly onto silica gel and purified by flash column chromatography (isocratic: $5: 95$ EtOAc : hexanes) to yield amino-iodinated product $20(59.4 \mathrm{mg}, 87 \%,>20: 1)$ as a pale yellow oil.

Rf: 0.30 (10\% EtOAc / hexanes).

${ }^{1} \mathrm{H}$ NMR (400 MHz, $\left.\mathrm{CDCl}_{3}\right): \delta=7.95-7.93(\mathrm{~m}, 2 \mathrm{H}), 7.50-7.46(\mathrm{~m}, 1 \mathrm{H})$, 7.42-7.38 $(\mathrm{m}, 2 \mathrm{H})$, $4.56(\mathrm{dd}, \mathrm{J}=11.3 \mathrm{~Hz}, 3.9 \mathrm{~Hz}, 1 \mathrm{H}), 4.47(\mathrm{~d}, \mathrm{~J}=8.6 \mathrm{~Hz}, 1 \mathrm{H}), 4.21(\mathrm{~d}, \mathrm{~J}=8.6 \mathrm{~Hz}, 1 \mathrm{H}), 2.54-$ $2.47(\mathrm{~m}, 1 \mathrm{H}), 2.09-2.05(\mathrm{~m}, 1 \mathrm{H}), 1.96-1.84(\mathrm{~m}, 3 \mathrm{H}), 1.65-1.59(\mathrm{~m}, 1 \mathrm{H}), 1.52-1.27(\mathrm{~m}, 2 \mathrm{H})$.

${ }^{13} \mathrm{C}$ NMR (100 MHz, $\left.\mathrm{CDCl}_{3}\right): \delta=163.05,131.58,128.55,128.44,127.81,76.44,75.06$, $44.26,36.88,36.33,27.08,22.42$.

HRMS (ESI-TOF) m/z: calc'd for $\mathrm{C}_{14} \mathrm{H}_{16} \mathrm{INO}[\mathrm{M}+\mathrm{H}]^{+} 342.0355$, found 342.0329 .

IR (film) cm-1: 3060, 2932, 2856, 1648, 1321, 1060, 1024, 693.

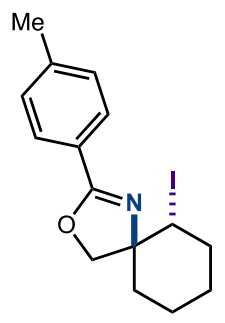

6-iodo-2-(p-tolyl)-3-oxa-1-azaspiro[4.5]dec-1-ene (21)

Imidate (S21) ( $46.3 \mathrm{mg}, 0.2 \mathrm{mmol}$ ) was subjected to GP3. Upon completion, the combined organic layer was concentrated and the residue was loaded directly onto silica gel and purified by flash column chromatography (isocratic: $5: 95$ EtOAc : hexanes) to yield amino-iodinated product $21(61.1 \mathrm{mg}, 87 \%,>20: 1)$ as a white solid.

Rf: 0.50 (20\% EtOAc / hexanes).

'1H NMR (400 MHz, $\left.\mathbf{C D C l}_{3}\right): \delta=7.83(\mathrm{~d}, \mathrm{~J}=8.2 \mathrm{~Hz}, 2 \mathrm{H}), 7.21(\mathrm{~d}, \mathrm{~J}=7.9 \mathrm{~Hz}, 2 \mathrm{H}), 4.54$ $(\mathrm{dd}, \mathrm{J}=11.3,3.9 \mathrm{~Hz}, 1 \mathrm{H}), 4.44(\mathrm{~d}, \mathrm{~J}=8.6 \mathrm{~Hz}, 1 \mathrm{H}), 4.18(\mathrm{~d}, \mathrm{~J}=8.6 \mathrm{~Hz}, 1 \mathrm{H}), 2.53-2.46(\mathrm{~m}$, 
$1 \mathrm{H}), 2.38(\mathrm{~s}, 3 \mathrm{H}), 2.10-2.04(\mathrm{~m}, 1 \mathrm{H}), 1.94-1.84(\mathrm{~m}, 3 \mathrm{H}), 1.64-1.59(\mathrm{~m}, 1 \mathrm{H}), 1.51-1.32(\mathrm{~m}$, $2 \mathrm{H})$.

${ }^{13} \mathrm{C}$ NMR (100 MHz, $\left.\mathrm{CDCl}_{3}\right): \delta=163.04,141.93,129.15,128.50,125.06,75.00,44.52$, $36.94,36.36,27.13,22.47,21.70$.

HRMS (ESI-TOF) m/z: calc'd for $\mathrm{C}_{15} \mathrm{H}_{18} \mathrm{INO}[\mathrm{M}+\mathrm{H}]^{+} 356.0511$, found 356.0496.

IR (film) cm': 3034, 2981, 2954, 1643, 1070, 827, 727.

MP: $89.0-91.0^{\circ} \mathrm{C}$.

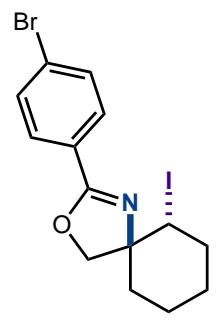

\section{2-(4-bromophenyl)-6-iodo-3-oxa-1-azaspiro[4.5]dec-1-ene (22)}

Imidate (S22) (59.2 mg, $0.2 \mathrm{mmol}$ ) was subjected to GP3. Upon completion, the combined organic layer was concentrated and the residue was loaded directly onto silica gel and purified by flash column chromatography (isocratic: $5: 95$ EtOAc : hexanes) to yield imidate 22 (59.7 mg, 71\%, >20:1) as a pale yellow oil.

Rf: 0.33 (10\% EtOAc / hexanes).

${ }^{1} \mathrm{H}$ NMR (400 MHz, CDCl$): ~ \delta=7.82-7.79(\mathrm{~m}, 2 \mathrm{H}), 7.55-7.53(\mathrm{~m}, 2 \mathrm{H}), 4.53(\mathrm{dd}, \mathrm{J}=11.3$, $3.9 \mathrm{~Hz}, 1 \mathrm{H}), 4.46(\mathrm{~d}, \mathrm{~J}=8.6 \mathrm{~Hz}, 1 \mathrm{H}), 4.20(\mathrm{~d}, \mathrm{~J}=8.6 \mathrm{~Hz}, 1 \mathrm{H}), 2.53-2.47(\mathrm{~m}, 1 \mathrm{H}), 2.10-$ $2.05(\mathrm{~m}, 1 \mathrm{H}), 1.94-1.84(\mathrm{~m}, 3 \mathrm{H}), 1.65-1.61(\mathrm{~m}, 1 \mathrm{H}), 1.52-1.30(\mathrm{~m}, 2 \mathrm{H})$.

${ }^{13} \mathrm{C}$ NMR (150 MHz, $\left.\mathrm{CDCl}_{3}\right): \delta=162.23,131.75,130.12,126.86,126.30,76.63,75.26$, 44.06, 36.90, 36.29, 27.09, 22.43.

HRMS (ESI-TOF) m/z: calc'd for $\mathrm{C}_{14} \mathrm{H}_{15} \mathrm{BrINO}[\mathrm{M}+\mathrm{H}]^{+} 419.9460$, found 419.9450 .

IR (film) cm': 3032, 2933, 2856, 1647, 1070, 726. 


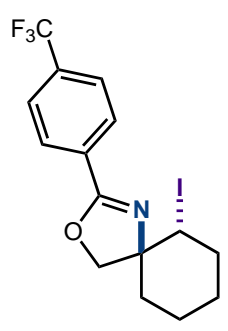

\section{6-iodo-2-(4-(trifluoromethyl)phenyl)-3-oxa-1-azaspiro[4.5]dec-1-ene (23)}

Imidate (S23) (57.1 mg, $0.2 \mathrm{mmol}$ ) was subjected to GP3. Upon completion, the combined organic layer was concentrated and the residue was loaded directly onto silica gel and purified by flash column chromatography (isocratic: $5: 95$ EtOAc : hexanes) to yield amino-iodinated product $23(67.1 \mathrm{mg}, 82 \%,>20: 1)$ as a white solid.

Rf: 0.33 (10\% EtOAc / hexanes).

${ }^{1} \mathrm{H}$ NMR $\left(600 \mathrm{MHz}, \mathrm{CDCl}_{3}\right): \delta=8.06(\mathrm{~d}, \mathrm{~J}=8.1 \mathrm{~Hz}, 2 \mathrm{H}), 7.67(\mathrm{~d}, \mathrm{~J}=8.2 \mathrm{~Hz}, 2 \mathrm{H}), 4.54$ (dd, J = 11.3, 3.9 Hz, 1H), $4.49(\mathrm{~d}, \mathrm{~J}=8.6 \mathrm{~Hz}, 1 \mathrm{H}), 4.24(\mathrm{~d}, \mathrm{~J}=8.4 \mathrm{~Hz}, 1 \mathrm{H}), 2.53-2.48(\mathrm{~m}$, $1 \mathrm{H}), 2.10-2.06(\mathrm{~m}, 1 \mathrm{H}), 1.94-1.86(\mathrm{~m}, 3 \mathrm{H}), 1.65-1.61(\mathrm{~m}, 1 \mathrm{H}), 1.51-1.44(\mathrm{~m}, 1 \mathrm{H}), 1.39-$ $1.33(\mathrm{~m}, 1 \mathrm{H})$.

${ }^{13} \mathrm{C}$ NMR (150 MHz, $\left.\mathbf{C D C l}_{3}\right): \delta=161.79,133.20$ (q, $\left.32.5 \mathrm{~Hz}\right), 131.29,128.95,125.43$ (q, 3.8 Hz), 123.93 (q, $272.5 \mathrm{~Hz}), 76.73,75.39,43.77,36.88,36.27,27.09,22.37$.

${ }^{19}$ F NMR (367 MHz, $\left.\mathrm{CDCl}_{3}\right):-62.98$.

HRMS (ESI-TOF) m/z: calc'd for $\mathrm{C}_{15} \mathrm{H}_{15} \mathrm{~F}_{3} \mathrm{INO}[\mathrm{M}+\mathrm{H}]^{+} 410.0229$, found 410.0214 .

IR (film) cm': 3003, 2955, 2864, 1646, 1318, 1073, 848, 676.

MP: $109.1-110.3^{\circ} \mathrm{C}$.

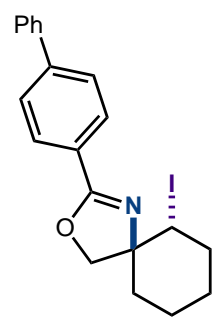

2-([1,1'-biphenyl]-4-yl)-6-iodo-3-oxa-1-azaspiro[4.5]dec-1-ene (24)

Imidate (S24) (58.7 mg, $0.2 \mathrm{mmol}$ ) was subjected to GP3. Upon completion, the combined organic layer was concentrated and the residue was loaded directly onto silica gel and 
purified by flash column chromatography (isocratic: $5: 95$, EtOAc : hexanes) to yield amino-iodinated product $22(63.4 \mathrm{mg}, 76 \%,>20: 1)$ as a white solid.

Rf: 0.39 (10\% EtOAc / hexanes).

${ }^{1} \mathrm{H}$ NMR $\left(600 \mathrm{MHz}, \mathrm{CDCl}_{3}\right): \delta=8.03-8.01(\mathrm{~m}, 2 \mathrm{H}), 7.65-7.61(\mathrm{~m}, 4 \mathrm{H}), 7.47-7.44(\mathrm{~m}, 2 \mathrm{H})$, 7.39-7.36 (m, 1H), $4.58(\mathrm{dd}, \mathrm{J}=11.3,3.9 \mathrm{~Hz}, 1 \mathrm{H}), 4.49(\mathrm{~d}, \mathrm{~J}=8.6 \mathrm{~Hz}, 1 \mathrm{H}), 4.23(\mathrm{~d}, \mathrm{~J}=$ $8.6 \mathrm{~Hz}, 1 \mathrm{H}), 2.54-2.50(\mathrm{~m}, 1 \mathrm{H}), 2.12-2.08(\mathrm{~m}, 1 \mathrm{H}), 1.97-1.88(\mathrm{~m}, 3 \mathrm{H}), 1.66-1.62(\mathrm{~m}, 1 \mathrm{H})$, $1.52-1.45(\mathrm{~m}, 1 \mathrm{H}), 1.41-1.34(\mathrm{~m}, 1 \mathrm{H})$.

${ }^{13} \mathrm{C}$ NMR (150 MHz, $\left.\mathrm{CDCl}_{3}\right): \delta=162.84,144.34,140.42,129.05,129.00,128.02,127.34$, $127.17,126.72,76.47,45.16,44.38,36.97,36.38,27.15,22.48$.

HRMS (ESI-TOF) m/z: calc'd for $\mathrm{C}_{20} \mathrm{H}_{20} \mathrm{INO}[\mathrm{M}+\mathrm{H}]^{+} 418.0668$, found 418.0651.

IR (film) cm': 3031, 3029, 2985, 2980, 1639, 1068, 825, 721.

MP: $81.3-83.2^{\circ} \mathrm{C}$.

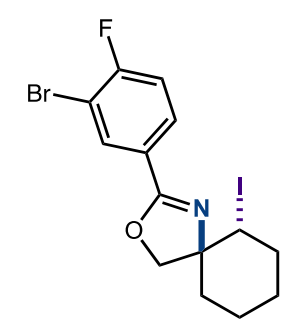

2-(3-bromo-4-fluorophenyl)-6-iodo-3-oxa-1-azaspiro[4.5]dec-1-ene (25)

Imidate (S25) (62.8 mg, $0.2 \mathrm{mmol}$ ) was subjected to GP3. Upon completion, the combined organic layer was concentrated and the residue was loaded directly onto silica gel and purified by flash column chromatography (isocratic: $5: 95$, EtOAc : hexanes) to yield amino-iodinated product $25(66.1 \mathrm{mg}, 75 \%, 13: 1)$ as a white solid.

Rf: 0.53 (15\% EtOAc / hexanes).

${ }^{1} \mathrm{H}$ NMR (400 MHz, $\left.\mathrm{CDCl}_{3}\right): \delta=8.18(\mathrm{dd}, \mathrm{J}=6.7,2.1 \mathrm{~Hz}, 1 \mathrm{H}), 7.86(\mathrm{qd}, \mathrm{J}=7.3,2.1 \mathrm{~Hz}$, $1 \mathrm{H}), 7.14(\mathrm{t}, \mathrm{J}=8.4 \mathrm{~Hz}, 1 \mathrm{H}), 4.51(\mathrm{~d}, \mathrm{~J}=11.3 \mathrm{~Hz}, 1 \mathrm{H}), 4.46(\mathrm{~d}, \mathrm{~J}=8.7 \mathrm{~Hz}, 1 \mathrm{H}), 4.20(\mathrm{~d}$, $\mathrm{J}=8.7 \mathrm{~Hz}, 1 \mathrm{H}), 2.53-2.46(\mathrm{~m}, 1 \mathrm{H}), 2.09-2.03(\mathrm{~m}, 1 \mathrm{H}), 1.93-1.83(\mathrm{~m}, 3 \mathrm{H}), 1.65-1.60(\mathrm{~m}$, $1 \mathrm{H}), 1.52-1.29(\mathrm{~m}, 2 \mathrm{H})$.

${ }^{13} \mathrm{C}$ NMR (100 MHz, $\left.\mathbf{C D C l}_{3}\right): \delta=161.18(\mathrm{~d}, 252.7 \mathrm{~Hz}), 160.96,134.05,129.50$ (d, J = 8.1 $\mathrm{Hz}), 125.53(\mathrm{~d}, \mathrm{~J}=3.7 \mathrm{~Hz}), 116.53(\mathrm{~d}, \mathrm{~J}=23.0 \mathrm{~Hz}), 109.36(\mathrm{~d}, \mathrm{~J}=21.6 \mathrm{~Hz}), 77.36,75.33$, $43.89,36.89,36.27,27.05,22.40$. 
${ }^{19}$ F NMR (367 MHz): $\delta=-102.42$.

HRMS (ESI-TOF) m/z: calc'd for $\mathrm{C}_{14} \mathrm{H}_{14} \mathrm{BrFINO}[\mathrm{M}+\mathrm{H}]^{+}$437.9366, found 437.9362 .

IR (film) cm$^{-1}:$ 3078, 2979, 2938, 2858, 1649, 1492, 830.

MP: $82-83^{\circ} \mathrm{C}$.

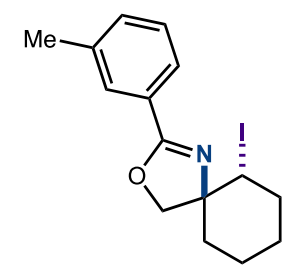

6-iodo-2-(m-tolyl)-3-oxa-1-azaspiro[4.5]dec-1-ene (26)

Imidate (S26) (46.3 mg, $0.2 \mathrm{mmol}$ ) was subjected to GP3. Upon completion, the combined organic layer was concentrated and the residue was loaded directly onto silica gel and purified by flash column chromatography (isocratic: $5: 95$, EtOAc : hexanes) to yield amino-iodinated product $26(63.4 \mathrm{mg}, 89 \%, 9: 1)$ as a pale yellow oil.

Rf: 0.55 (15\% EtOAc / hexanes).

${ }^{1} \mathrm{H}$ NMR (400 MHz, $\left.\mathrm{CDCl}_{3}\right): \delta=7.78(\mathrm{~m}, 1 \mathrm{H}), 7.74-7.71(\mathrm{~m}, 1 \mathrm{H}), 7.30-7.28(\mathrm{~m}, 2 \mathrm{H}), 4.55$ $(\mathrm{dd}, \mathrm{J}=11.4,3.9 \mathrm{~Hz}, 1 \mathrm{H}), 4.46(\mathrm{~d}, \mathrm{~J}=8.6 \mathrm{~Hz}, 1 \mathrm{H}), 4.20(\mathrm{~d}, \mathrm{~J}=8.7 \mathrm{~Hz}, 1 \mathrm{H}), 2.54-2.48(\mathrm{~m}$, $1 \mathrm{H}), 2.38(\mathrm{~s}, 3 \mathrm{H}), 2.10-2.04(\mathrm{~m}, 1 \mathrm{H}), 1.96-1.84(\mathrm{~m}, 3 \mathrm{H}), 1.67-1.60(\mathrm{~m}, 1 \mathrm{H}), 1.52-1.31(\mathrm{~m}$, $2 \mathrm{H})$.

${ }^{13} \mathrm{C}$ NMR (100 MHz, $\left.\mathbf{C D C l}_{3}\right): \delta=163.16,138.23,132.38,129.09,128.37,127.75,125.65$, $76.32,75.07,44.35,36.98,36.41,27.18,22.47,21.37$.

HRMS (ESI-TOF) m/z: calc'd for $\mathrm{C}_{15} \mathrm{H}_{18} \mathrm{INO}[\mathrm{M}+\mathrm{H}]^{+} 356.0511$, found 356.0501 .

IR (film) cm': 3039, 2932, 2857, 1647, 1063, 712.

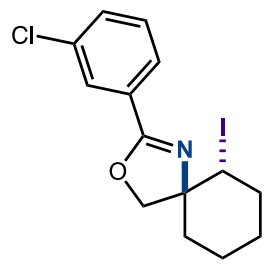

\section{2-(3-chlorophenyl)-6-iodo-3-oxa-1-azaspiro[4.5]dec-1-ene (27)}

Imidate (S27) (50.3 mg, $0.2 \mathrm{mmol}$ ) was subjected to GP3. Upon completion, the combined organic layer was concentrated and the residue was loaded directly onto silica gel and 
purified by flash column chromatography (isocratic: $5: 95$ EtOAc : hexanes) to yield amino-iodinated 27 (75.1 mg, 83\%, 9:1) as a pale yellow solid.

Rf: 0.53 (15\% EtOAc / hexanes).

${ }^{1} \mathrm{H}$ NMR (400 MHz, $\left.\mathbf{C D C l}_{3}\right): \delta=7.95-7.94(\mathrm{~m}, 1 \mathrm{H}), 7.83-7.81(\mathrm{~m}, 1 \mathrm{H}), 7.46-7.43(\mathrm{~m}, 1 \mathrm{H})$, 7.36-7.32 (m, 1H), $4.53(\mathrm{dd}, \mathrm{J}=11.3,3.9 \mathrm{~Hz}, 1 \mathrm{H}), 4.47(\mathrm{~d}, \mathrm{~J}=8.7 \mathrm{~Hz}, 1 \mathrm{H}), 4.21(\mathrm{~d}, \mathrm{~J}=$ $8.7 \mathrm{~Hz}, 1 \mathrm{H}), 2.53-2.47(\mathrm{~m}, 1 \mathrm{H}), 2.09-2.04(\mathrm{~m}, 1 \mathrm{H}), 1.93-1.83(\mathrm{~m}, 3 \mathrm{H}), 1.65-1.61(\mathrm{~m}, 1 \mathrm{H})$, $1.53-1.31(\mathrm{~m}, 2 \mathrm{H})$.

${ }^{13} \mathrm{C}$ NMR (100 MHz, $\left.\mathrm{CDCl}_{3}\right): \delta=161.84,134.56,131.61,129.76,129.65,128.65,126.64$, 76.70, 75.24, 43.92, 36.87, 36.26, 27.03, 22.38 .

HRMS (ESI-TOF) m/z: calc'd for $\mathrm{C}_{14} \mathrm{H}_{15} \mathrm{CIINO}[\mathrm{M}+\mathrm{H}]^{+} 375.9965$, found 375.9956.

IR (film) cm': 3078, 2944, 2921, 2853, 1643, 1315, 973, 754.

MP: $96.3-97.5^{\circ} \mathrm{C}$.

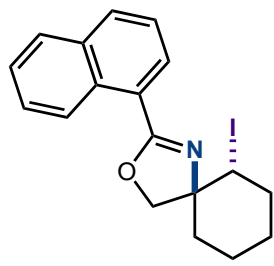

\section{6-iodo-2-(naphthalen-1-yl)-3-oxa-1-azaspiro[4.5]dec-1-ene (28)}

Imidate (S28) ( $83.5 \mathrm{mg}, 0.2 \mathrm{mmol}$ ) was subjected to GP3. Upon completion, the combined organic layer was concentrated and the residue was loaded directly onto silica gel and purified by flash column chromatography (isocratic: $5: 95$, EtOAc : hexanes) to yield amino-iodinated product $28(78.3 \mathrm{mg}, 70 \%, 9: 1)$ as a white solid.

Rf: 0.66 (15\% EtOAc / hexanes).

${ }^{1} \mathrm{H}$ NMR (400 MHz, $\left.\mathrm{CDCl}_{3}\right): \delta=9.08-9.06(\mathrm{~m}, 1 \mathrm{H}), 8.08-8.06(\mathrm{~m}, 1 \mathrm{H})$, 7.97-7.95 $(\mathrm{m}, 1 \mathrm{H})$, 7.88-7.86 (m, 1H), 7.63-7.58 (m, 1H), 7.54-7.47 (m, 2H), $4.67(\mathrm{dd}, \mathrm{J}=11.0,3.9 \mathrm{~Hz}, 1 \mathrm{H})$, $4.51(\mathrm{~d}, \mathrm{~J}=8.6 \mathrm{~Hz}, 1 \mathrm{H}), 4.26(\mathrm{~d}, \mathrm{~J}=8.6 \mathrm{~Hz}, 1 \mathrm{H}), 2.60-2.53(\mathrm{~m}, 1 \mathrm{H}), 2.22-2.16(\mathrm{~m}, 1 \mathrm{H})$ 2.06-1.91 (m, 3H), 1.72-1.65 (m, 1H), 1.58-1.41 (m, 2H).

${ }^{13} \mathrm{C} \mathrm{NMR}\left(\mathbf{1 0 0} \mathrm{MHz}, \mathrm{CDCl}_{3}\right): \delta=163.01,133.86,132.00,131.28,129.08,128.48,127.39$, $126.64,126.22,124.87,124.77,77.36,75.90,44.39,36.98,36.26,26.94,22.45$.

HRMS (ESI-TOF) m/z: calc'd for $\mathrm{C}_{18} \mathrm{H}_{18} \mathrm{INO}[\mathrm{M}+\mathrm{H}]^{+} 392.0511$, found 392.0497.

IR (film) cm': 3091, 3049, 2968, 2933, 2850, 1640, 1509, 1121, 1005, 774.

MP: $99-100^{\circ} \mathrm{C}$. 


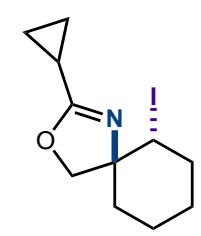

2-cyclopropyl-6-iodo-3-oxa-1-azaspiro[4.5]dec-1-ene (29)

Imidate (S29) (66.3 mg, $0.2 \mathrm{mmol}$ ) was subjected to GP3. Upon completion, the combined organic layer was concentrated and the residue was loaded directly onto silica gel and purified by flash column chromatography (isocratic: $10: 90$, EtOAc : hexanes) to yield amino-iodinated product $29(48.2 \mathrm{mg}, 80 \%, 19: 1)$ as a colorless oil.

Rf: 0.43 (15\% EtOAc / hexanes).

${ }^{1} \mathrm{H}$ NMR $\left(700 \mathrm{MHz}, \mathrm{CDCl}_{3}\right): \delta=4.39(\mathrm{dd}, \mathrm{J}=11.4,3.9 \mathrm{~Hz}, 1 \mathrm{H}), 4.20(\mathrm{~d}, \mathrm{~J}=8.6 \mathrm{~Hz}, 1 \mathrm{H})$, $3.94(\mathrm{~d}, \mathrm{~J}=8.6 \mathrm{~Hz}, 1 \mathrm{H}), 2.41(\mathrm{dq}, \mathrm{J}=12.9,4.1 \mathrm{~Hz}, 1 \mathrm{H}), 1.96-1.93(\mathrm{~m}, 1 \mathrm{H}), 1.81-1.75(\mathrm{~m}$, $3 \mathrm{H}), 1.61-1.53(\mathrm{~m}, 2 \mathrm{H}), 1.40-1.35(\mathrm{~m}, 1 \mathrm{H}), 1.28-1.23(\mathrm{~m}, 1 \mathrm{H}), 0.96-0.95(\mathrm{~m}, 2 \mathrm{H}), 0.84-$ $0.83(\mathrm{~m}, 2 \mathrm{H})$.

${ }^{13} \mathrm{C}$ NMR (175 MHz, $\left.\mathrm{CDCl}_{3}\right): \delta=168.34,76.13,74.27,44.96,36.97,22.59,8.75,7.38$, 7.11 .

HRMS (ESI-TOF) m/z: calc'd for $\mathrm{C}_{11} \mathrm{H}_{16} \mathrm{INO}[\mathrm{M}+\mathrm{H}]^{+} 306.0355$, found 306.0344 .

IR (film) $\mathbf{c m}^{-1}:$ 2930, 2857, 1728, 1658, 1171.

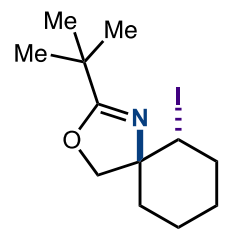

\section{2-(tert-butyl)-6-iodo-3-oxa-1-azaspiro[4.5]dec-1-ene (30)}

Imidate (S30) (39.5 mg, $0.2 \mathrm{mmol}$ ) was subjected to GP3. Upon completion, the combined organic layer was concentrated and the residue was loaded directly onto silica gel and purified by flash column chromatography (isocratic: $10: 90$, EtOAc : hexanes) to yield amino-iodinated product $\mathbf{3 0}(55.1 \mathrm{mg}, 86 \%, 12: 1)$ as a colorless oil.

Rf: 0.61 (15\% EtOAc / hexanes). 
${ }^{1} \mathrm{H}$ NMR (400 MHz, $\left.\mathrm{CDCl}_{3}\right): \delta=4.47(\mathrm{dd}, \mathrm{J}=12.3,4.0 \mathrm{~Hz}, 1 \mathrm{H}), 4.27(\mathrm{~d}, \mathrm{~J}=8.6 \mathrm{~Hz}, 1 \mathrm{H})$, $4.00(\mathrm{~d}, \mathrm{~J}=8.6 \mathrm{~Hz}, 1 \mathrm{H}), 2.48-2.41(\mathrm{~m}, 1 \mathrm{H}), 1.95-1.76(\mathrm{~m}, 4 \mathrm{H}), 1.56-1.52(\mathrm{~m}, 1 \mathrm{H}), 1.43-$ $1.27(\mathrm{~m}, 2 \mathrm{H}), 1.22(\mathrm{~s}, 9 \mathrm{H})$.

${ }^{13} \mathrm{C}$ NMR (100 MHz, $\left.\mathrm{CDCl}_{3}\right): \delta=173.20,75.50,74.34,44.94,37.16,36.82,33.31,28.00$, 27.79, 22.62.

HRMS (ESI-TOF) m/z: calc'd for $\mathrm{C}_{12} \mathrm{H}_{20} \mathrm{INO}[\mathrm{M}+\mathrm{H}]^{+} 322.0668$, found 322.0661 .

IR (film) cm$^{-1}:$ 2954, 2930, 2858, 1730, 1655, 1125, 1097, 990.

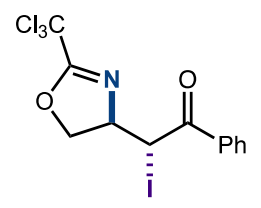

\section{2-iodo-1-phenyl-2-(2-(trichloromethyl)-4,5-dihydrooxazol-4-yl)ethan-1-one (34)}

Imidate (33) $(61.7 \mathrm{mg}, 0.2 \mathrm{mmol})$ was subjected to GP3 with the following modifications: $\mathrm{Nal}$ ( 3 equiv) was used in place of $\mathrm{I}_{2}$, and $\mathrm{Phl}(\mathrm{OAc})_{2}$ (3.5 equiv) was used. Upon completion, the combined organic layer was concentrated and the yield was determined by ${ }^{1} \mathrm{H}$-NMR using dichloroethane as an internal standard (59\%, 1.5:1). Compound 34 was isolated using preparatory thin-layer chromatography (10:90 EtOAc : hexanes) as a colorless oil.

Rf: 0.36 (15\% EtOAc / hexanes).

${ }^{1} \mathrm{H}$ NMR $\left(600 \mathrm{MHz}, \mathrm{CDCl}_{3}\right): \delta=7.99-7.97(\mathrm{~m}, 2 \mathrm{H}), 7.63-7.61(\mathrm{~m}, 1 \mathrm{H}), 7.52-7.49(\mathrm{~m}, 2 \mathrm{H})$, $6.06(\mathrm{~d}, \mathrm{~J}=2.9 \mathrm{~Hz}, 1 \mathrm{H}), 5.03(\mathrm{dd}, \mathrm{J}=9.8,9.8 \mathrm{~Hz}, 1 \mathrm{H}), 4.71(\mathrm{dd}, \mathrm{J}=9.8,7.6 \mathrm{~Hz}, 1 \mathrm{H}), 4.29$ (ddd, $\mathrm{J}=9.6,7.8,3.0 \mathrm{~Hz}, 1 \mathrm{H}$ ).

${ }^{13} \mathrm{C}$ NMR $\left(150 \mathrm{MHz}, \mathrm{CDCl}_{3}\right): \delta=193.89,164.82,134.40,133.36,129.09,128.97,86.64$, $78.08,67.15,33.23$.

HRMS (ESI-TOF) m/z: calc'd for $\mathrm{C}_{12} \mathrm{H}_{9} \mathrm{Cl}_{3} \mathrm{INO}_{2}[\mathrm{M}+\mathrm{H}]^{+} 431.8822$, found 431.8817 .

IR (film) cm-1: 3061, 2980, 2924, 2853, 1770, 1709, 1669, 1233, 986, 795. 


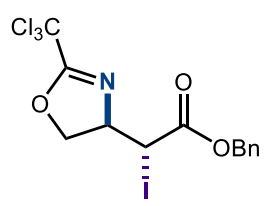

benzyl 2-iodo-2-(2-(trichloromethyl)-4,5-dihydrooxazol-4-yl)acetate (36)

Imidate 35 (67.7 $\mathrm{mg}, 0.2 \mathrm{mmol})$ was subjected to GP3 with the following changes: $\mathrm{Phl}(\mathrm{OAc})_{2}(257.7 \mathrm{mg}, 0.8 \mathrm{mmol})$. In addition, the reaction time extended to 4 hours. Upon completion, the combined organic layer was concentrated and the yield was determined by ${ }^{1} \mathrm{H}$-NMR using dichloroethane as an internal standard $(55 \%, 1: 1)$. Compound $\mathbf{3 6}$ was isolated using preparatory thin-layer chromatography $(10: 90 \mathrm{EtOAc}$ : hexanes) as a yellow oil.

Rf: 0.21 (10\% EtOAc/hexanes)

${ }^{1} \mathbf{H}$ NMR $\left(600 \mathrm{MHz}, \mathbf{C D C l}_{3}\right)$ : Diastereomer $\mathbf{A} \delta=7.42-7.35(\mathrm{~m}, 5 \mathrm{H}), 5.23(\mathrm{~d}, \mathrm{~J}=$ $12.1 \mathrm{~Hz}, 1 \mathrm{H}), 5.19(\mathrm{~d}, \mathrm{~J}=12.1 \mathrm{~Hz}, 1 \mathrm{H}), 4.95(\mathrm{~d}, \mathrm{~J}=4.4 \mathrm{~Hz}, 1 \mathrm{H}), 4.81(\mathrm{dd}, \mathrm{J}=9.7 \mathrm{~Hz}$, $4.7 \mathrm{~Hz}, 1 \mathrm{H}), 4.63(\mathrm{dd}, \mathrm{J}=9.7 \mathrm{~Hz}, 7.6 \mathrm{~Hz}, 1 \mathrm{H}), 4.34$ (ddd, J = 9.7 Hz, 7.5 Hz, $4.4 \mathrm{~Hz}$, $1 \mathrm{H})$.

${ }^{13} \mathbf{C}$ NMR (150 MHz, $\left.\mathbf{C D C l}_{3}\right): \delta=$ Diastereomer $\mathbf{A} \delta=169.69,164.82,134.87,128.85$, $128.81,128.39,68.13,67.47,26.81$.

HRMS (ESI-TOF) m/z: calc'd for $\mathrm{C}_{13} \mathrm{H}_{11} \mathrm{Cl}_{3} \mathrm{INO}_{3}[\mathrm{M}+\mathrm{Na}]^{+} 483.8741$, found 483.8734 .

IR (neat) cm$^{-1}:$ 2934, 1719, 1702, 1658, 1498, 1455, 1380, 1326, 1212, 1188.

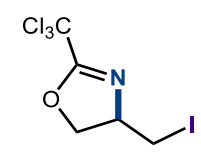

\section{4-(iodomethyl)-2-(trichloromethyl)-4,5-dihydrooxazole $(38,40)$}

Procedure A: Imidate 37 (55.0 $\mathrm{mg}, 0.2 \mathrm{mmol}$ ) was subjected to GP3 with the following changes: Nal (90 mg, $0.4 \mathrm{mmol})$. Upon completion, the combined organic layer was concentrated in vacuo before purification by flash chromatography (1\% EtOAc/hexanes with $\left.1 \% \mathrm{Et}_{3} \mathrm{~N}\right)$ to afford amino-iodinated product $38(49.3 \mathrm{mg}, 75 \%)$ as a yellowish solid.

Procedure B: Imidate 39 (55.3 $\mathrm{mg}, 0.2 \mathrm{mmol}$ ) was subjected to GP3 with the following changes: Nal (90 mg, $0.4 \mathrm{mmol})$. Upon completion, the combined organic layer was concentrated in vacuo before purification by flash chromatography (1\% EtOAc/hexanes with $\left.1 \% \mathrm{Et}_{3} \mathrm{~N}\right)$ to afford amino-iodinated product $40(46.0 \mathrm{mg}, 70 \%)$ as a yellowish solid.

Rf: 0.37 (20\% EtOAc/hexanes) 
${ }^{1} \mathrm{H}$ NMR $\left(400 \mathrm{MHz}, \mathrm{CDCl}_{3}\right): \delta=4.72(\mathrm{dd}, \mathrm{J}=9.2 \mathrm{~Hz}, 8.8 \mathrm{~Hz}, 1 \mathrm{H}), 4.58-4.51(\mathrm{~m}, 1 \mathrm{H})$, $4.42(\mathrm{dd}, \mathrm{J}=8.8 \mathrm{~Hz}, \mathrm{~J}=7.3 \mathrm{~Hz}, 1 \mathrm{H}), 3.46(\mathrm{dd}, \mathrm{J}=10.4 \mathrm{~Hz}, 3.6 \mathrm{~Hz}, 1 \mathrm{H}), 3.32(\mathrm{dd}, \mathrm{J}=$ $10.5 \mathrm{~Hz}, 7.3 \mathrm{~Hz}, 1 \mathrm{H})$.

${ }^{13} \mathrm{C}$ NMR (100 MHz, $\left.\mathrm{CDCl}_{3}\right): \delta=164.31,86.64,67.00,31.07,8.74$.

HRMS (ESI-TOF) m/z: calc'd for $\mathrm{C}_{5} \mathrm{H}_{5} \mathrm{Cl}_{3} \mathrm{INO}[\mathrm{M}+\mathrm{Na}]^{+} 349.8374$, found 349.8396 .

IR (neat) cm$^{-1}:$ 3007, 2962, 2904, 1701, 1656, 1466, 1414, 1355, 1329.

MP: $48.7^{\circ} \mathrm{C}-50.6^{\circ} \mathrm{C}$.

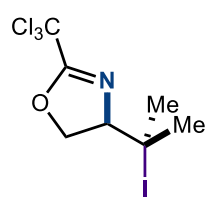

4-(2-iodopropan-2-yl)-2-(trichloromethyl)-4,5-dihydrooxazole (42)

Imidate 41 (46.5 mg, $0.2 \mathrm{mmol}$ ) was subjected to GP3 with the following changes: Nal (60 $\mathrm{mg}, 0.4 \mathrm{mmol})$. In addition, the reaction time shortened to 20 minutes. Upon completion, the combined organic layer was concentrated in vacuo before purification by flash chromatography $\left(1: 98: 1 \mathrm{EtOAc}:\right.$ hexanes : $\left.\mathrm{Et}_{3} \mathrm{~N}\right)$ to afford amino-iodinated product $42(32.1 \mathrm{mg}, 45 \%)$ as a white solid.

Rf: 0.26 (5\% EtOAc/hexanes)

${ }^{1} \mathrm{H}$ NMR $\left(400 \mathrm{MHz}, \mathrm{CDCl}_{3}\right): \delta=4.72(\mathrm{dd}, \mathrm{J}=10.0 \mathrm{~Hz}, \mathrm{~J}=9.3 \mathrm{~Hz}, 1 \mathrm{H}), 4.62(\mathrm{dd}, \mathrm{J}=9.2$ $\mathrm{Hz}, \mathrm{J}=7.6 \mathrm{~Hz}, 1 \mathrm{H}), 4.19(\mathrm{dd}, \mathrm{J}=9.8 \mathrm{~Hz}, \mathrm{~J}=7.5 \mathrm{~Hz}, 1 \mathrm{H}), 2.04(\mathrm{~s}, 3 \mathrm{H}), 1.95(\mathrm{~s}, 3 \mathrm{H})$.

${ }^{13} \mathrm{C}$ NMR (100 MHz, $\left.\mathrm{CDCl}_{3}\right): \delta=163.92,78.64,76.33,50.54,35.17,32.10$.

HRMS (ESI-TOF) m/z: calc'd for $\mathrm{C}_{7} \mathrm{H}_{9} \mathrm{Cl}_{3} \mathrm{INO}[\mathrm{M}+\mathrm{Na}]^{+} 377.8687$, found 377.8703 .

IR (neat) cm$^{-1}:$ 2989, 2966, 2924, 2866, 1763, 1662, 1451.

MP: $87.6^{\circ} \mathrm{C}-89.2^{\circ} \mathrm{C}$. 
VII. Post-Synthetic Functionalization Characterization

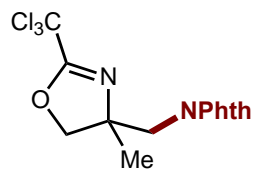

2-((4-methyl-2-(trichloromethyl)-4,5-dihydrooxazol-4-yl)methyl)isoindoline-1,3dione (43)

To a 2-dram vial equipped with a PTFE septum cap and magnetic stir bar was added oxazoline 17 (40 mg, $0.117 \mathrm{mmol}$ ) and KPhthalimide $(43.3 \mathrm{mg}, 0.234 \mathrm{mmol})$. Dry DMF $(1.67 \mathrm{~mL})$ was added to the vial, and the reaction was stirred at $100^{\circ} \mathrm{C}$ for $24 \mathrm{~h}$. Upon completion, the solution was cooled to room temperature then concentrated in vacuo. The solution was then diluted with $\mathrm{CH}_{2} \mathrm{Cl}_{2}$, and washed with $1 \mathrm{M} \mathrm{NaOH}(2 \mathrm{x})$, and brine (1x). The combined organic layer was concentrated in vacuo before purification by flash chromatography (15\% EtOAc/hexanes) to afford product $43(27.1 \mathrm{mg}, 64 \%)$ as a white solid.

Rf: 0.13 (15\% EtOAc/hexanes)

${ }^{1} \mathrm{H}$ NMR (400 MHz, $\left.\mathrm{CDCl}_{3}\right): \delta=7.89-7.85(\mathrm{~m}, 2 \mathrm{H}), 7.78-7.72(\mathrm{~m}, 2 \mathrm{H}), 4.97(\mathrm{~d}, \mathrm{~J}=8.9$ $\mathrm{Hz}, 1 \mathrm{H}), 4.21(\mathrm{~d}, \mathrm{~J}=9.0 \mathrm{~Hz}, 1 \mathrm{H}), 3.95(\mathrm{AB} \mathrm{q}, \mathrm{J}=11.1,2 \mathrm{H}), 1.50(\mathrm{~s}, 3 \mathrm{H})$.

${ }^{13} \mathrm{C}$ NMR (400 MHz, $\left.\mathrm{CDCl}_{3}\right): \delta=168.56,162.69,134.38,131.93,123.64,80.04,72.76$, $45.61,29.83,24.29$.

HRMS (ESI-TOF) m/z: calc'd for $\mathrm{C}_{14} \mathrm{H}_{11} \mathrm{Cl}_{3} \mathrm{~N}_{2} \mathrm{O}_{3}[\mathrm{M}+\mathrm{Na}]^{+} 382.9727$, found 382.9724 .

IR (film) cm-1: 3185, 3059, 2919, 2850, 1773, 1706, 1661, 1604, 1467, 1051, 727, 712.

MP: $156.0^{\circ} \mathrm{C}-159.6^{\circ} \mathrm{C}$

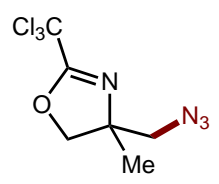

\section{4-(azidomethyl)-4-methyl-2-(trichloromethyl)-4,5-dihydrooxazole (44)}

To a 2-dram vial equipped with a PTFE septum cap and magnetic stir bar was added oxazoline 17 (68.5 mg, $0.2 \mathrm{mmol}$ ) and sodium azide (26 mg, $0.4 \mathrm{mmol})$. Dry DMF (1 mL) was added to the vial, and the reaction was stirred at $80^{\circ} \mathrm{C}$ for $24 \mathrm{~h}$. Upon completion, the solution was cooled to $\mathrm{RT}$ then dumped into $\mathrm{H}_{2} \mathrm{O}$. The aqueous layer was extracted $3 \mathrm{x}$ with $\mathrm{CH}_{2} \mathrm{Cl}_{2}(5 \mathrm{~mL})$. The organic layer was washed $5 x$ with $\mathrm{H}_{2} \mathrm{O}(5 \mathrm{~mL})$. The combined organic layer was concentrated in vacuo before purification by flash chromatography 
(isocratic: $15: 85$, EtOAc : hexanes) to afford product $\mathbf{4 4}(44.7 \mathrm{mg}, 87 \%)$ as a colorless oil.

Rf: 0.25 (10\% EtOAc / hexanes)

${ }^{1} \mathrm{H}$ NMR (400 MHz, $\left.\mathrm{CDCl}_{3}\right): \delta=4.57(\mathrm{~d}, \mathrm{~J}=8.6 \mathrm{~Hz}, 1 \mathrm{H}), 4.23(\mathrm{~d}, \mathrm{~J}=8.6 \mathrm{~Hz}, 1 \mathrm{H}), 3.57$ $(\mathrm{d}, \mathrm{J}=12.5 \mathrm{~Hz}, 1 \mathrm{H}), 3.27(\mathrm{~d}, \mathrm{~J}=12.5 \mathrm{~Hz}, 1 \mathrm{H}), 1.41(\mathrm{~s}, 3 \mathrm{H})$.

${ }^{13} \mathrm{C}$ NMR (100 MHz, $\left.\mathrm{CDCl}_{3}\right): \delta=163.11,86.42,78.65,72.32,58.42,24.17$.

HRMS (ESI-TOF) m/z: calc'd for $\mathrm{C}_{6} \mathrm{H}_{7} \mathrm{Cl}_{3} \mathrm{~N}_{4} \mathrm{O}[\mathrm{M}+\mathrm{Na}]^{+}$278.9583, found 278.9563.

IR (film) cm$^{-1}:$ 2097, 1658, 998.

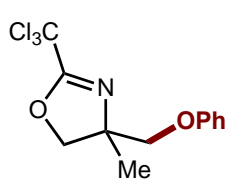

\section{4-methyl-4-(phenoxymethyl)-2-(trichloromethyl)-4,5-dihydrooxazole (45)}

To a 2-dram vial equipped with a PTFE septum cap and magnetic stir bar was added oxazoline 17 (68.5 mg, $0.2 \mathrm{mmol}$ ) and NaOPh (46.4 mg, $0.4 \mathrm{mmol})$. Dry DMF (1 mL) was added to the vial, and the reaction was stirred at $100^{\circ} \mathrm{C}$ for $15 \mathrm{~h}$. Upon completion, the solution was cooled to room temperature then dumped into $\mathrm{H}_{2} \mathrm{O}$. The aqueous layer was extracted $3 x$ with $\mathrm{CH}_{2} \mathrm{Cl}_{2}(5 \mathrm{~mL})$. The organic layer was washed with $10 \% \mathrm{NaOH}(\mathrm{aq})$ followed by $5 \mathrm{x} \mathrm{H}_{2} \mathrm{O}(5 \mathrm{~mL})$. The combined organic layer was concentrated in vacuo before purification by flash chromatography (isocratic: $20: 80$, EtOAc : hexanes) to afford product $45(57.4 \mathrm{mg}, 93 \%)$ as a white solid.

Rf: 0.27 (10\% EtOAc / hexanes).

${ }^{1} \mathrm{H}$ NMR (400 MHz, $\left.\mathrm{CDCl}_{3}\right): \delta=7.30-7.27(\mathrm{~m}, 2 \mathrm{H}), 6.99-6.95(\mathrm{~m}, 1 \mathrm{H}), 6.91-6.88(\mathrm{~m}$, $2 \mathrm{H}), 4.78(\mathrm{~d}, \mathrm{~J}=8.5 \mathrm{~Hz}, 1 \mathrm{H}), 4.32(\mathrm{~d}, \mathrm{~J}=8.5 \mathrm{~Hz}, 1 \mathrm{H}), 4.03(\mathrm{~d}, \mathrm{~J}=9.2 \mathrm{~Hz}, 1 \mathrm{H}), 3.96(\mathrm{~d}, \mathrm{~J}$ $=9.2 \mathrm{~Hz}, 1 \mathrm{H}), 1.52(\mathrm{~s}, 3 \mathrm{H})$.

${ }^{13} \mathrm{C}$ NMR (100 MHz, $\left.\mathrm{CDCl}_{3}\right): \delta=162.89,158.63,129.65,121.46,114.73,78.96,77.35$, $72.70,71.78,23.35$.

HRMS (ESI-TOF) m/z: calc'd for $\mathrm{C}_{12} \mathrm{H}_{12} \mathrm{Cl}_{3} \mathrm{NO}_{2}[\mathrm{M}+\mathrm{Na}]^{+} 329.9800$, found 329.9822 .

IR (film) cm$^{-1}:$ 2976, 2930, 2871, 1665, 1236, 1080.

MP: $111-112^{\circ} \mathrm{C}$. 


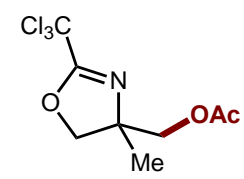

\section{(4-methyl-2-(trichloromethyl)-4,5-dihydrooxazol-4-yl)methyl acetate (46)}

To a 2-dram vial equipped with a PTFE septum cap and magnetic stir bar was added oxazoline 17 (68.5 mg, $0.2 \mathrm{mmol})$ and KOAc (78.5 mg, $0.8 \mathrm{mmol})$. Dry DMF (1 mL) was added to the vial, and the reaction was stirred at $100^{\circ} \mathrm{C}$ for $48 \mathrm{~h}$. Upon completion, the solution was cooled to room temperature then dumped into $\mathrm{H}_{2} \mathrm{O}$. The aqueous layer was extracted $3 x$ with $\mathrm{CH}_{2} \mathrm{Cl}_{2}(5 \mathrm{~mL})$. The organic layer was washed $5 x$ with $\mathrm{H}_{2} \mathrm{O}(5 \mathrm{~mL})$. The combined organic layer was concentrated in vacuo before purification by flash chromatography (isocratic: $20: 80$, EtOAc : hexanes) to afford product 46 (54.9 mg, $100 \%)$ as a colorless oil.

Rf: 0.25 (10\% EtOAc / hexanes).

${ }^{1} \mathrm{H}$ NMR (400 MHz, $\left.\mathrm{CDCl}_{3}\right): \delta=4.57(\mathrm{~d}, \mathrm{~J}=8.6 \mathrm{~Hz}, 1 \mathrm{H}), 4.24(\mathrm{~d}, \mathrm{~J}=8.6 \mathrm{~Hz}, 1 \mathrm{H}), 4.16$ (d, J = 11.4 Hz, 1H), 4.09 (d, J = 11.4 Hz, 1H), $2.06(\mathrm{~s}, 3 \mathrm{H}), 1.42(\mathrm{~s}, 3 \mathrm{H})$.

${ }^{13} \mathrm{C}$ NMR (100 MHz, $\left.\mathrm{CDCl}_{3}\right): \delta=170.69,169.82,86.54,78.46,77.36,71.09,68.58,23.06$, 20.80 .

HRMS (ESI-TOF) m/z: calc'd for $\mathrm{C}_{8} \mathrm{H}_{10} \mathrm{Cl}_{3} \mathrm{NO}_{3}[\mathrm{M}+\mathrm{Na}]^{+} 295.9600$, found 295.9620.

IR (film) cm$^{-1}: 1735,1660,1160$.

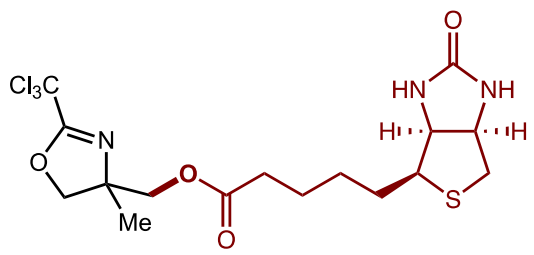

(4-methyl-2-(trichloromethyl)-4,5-dihydrooxazol-4-yl)methyl 5-((3aS,4S,6a R)-2oxohexahydro-1H-thieno[3,4- $d$ ] imidazol-4-yl)pentanoate (47)

To a 2-dram vial equipped with a PTFE septum cap and magnetic stir bar was added oxazoline 17 (68.5 mg, $0.2 \mathrm{mmol}$ ), D-biotin $\left(97.7 \mathrm{mg}, 0.4 \mathrm{mmol}\right.$ ), and $\mathrm{K}_{2} \mathrm{CO}_{3}$ (110.6 $\mathrm{mg}$, $0.8 \mathrm{mmol})$. Dry DMF $(1 \mathrm{~mL})$ was added to the vial, and the reaction was stirred at $110^{\circ} \mathrm{C}$ for $7 \mathrm{~h}$. Upon completion, the solution was cooled to room temperature and concentrated in vacuo before purification by flash chromatography (isocratic: $5: 95, \mathrm{MeOH}: \mathrm{CH}_{2} \mathrm{Cl}_{2}$ ) to afford product 47 (69.7 mg, 76\%, 1:1 dr) as a pale yellow oil. 
Rf: $0.22\left(5 \% \mathrm{MeOH} / \mathrm{CH}_{2} \mathrm{Cl}_{2}\right)$.

${ }^{1} \mathbf{H}$ NMR (600 MHz, $\left.\mathbf{C D C l}_{3}\right):(1: 1$ mixture of two diastereomers) $\delta=5.55(\mathrm{bs}, 1 \mathrm{H}), 5.50$ (bs, $1 \mathrm{H}), 5.09$ (bs, 2H), $4.57(\mathrm{~d}, \mathrm{~J}=8.6 \mathrm{~Hz}, 1 \mathrm{H}), 4.57(\mathrm{~d}, \mathrm{~J}=8.6 \mathrm{~Hz}, 1 \mathrm{H}), 4.52-4.50(\mathrm{~m}$, 2H), 4.32-4.30 (m, 2H), $4.25(\mathrm{~d}, \mathrm{~J}=8.6 \mathrm{~Hz}, 1 \mathrm{H}), 4.25(\mathrm{~d}, \mathrm{~J}=8.6 \mathrm{~Hz}, 1 \mathrm{H}), 4.18(\mathrm{~d}, \mathrm{~J}=11.4$ $\mathrm{Hz}, 1 \mathrm{H}), 4.17(\mathrm{~d}, \mathrm{~J}=11.4 \mathrm{~Hz}, 1 \mathrm{H}), 4.13(\mathrm{~d}, \mathrm{~J}=11.4 \mathrm{~Hz}, 1 \mathrm{H}), 4.12(\mathrm{~d}, \mathrm{~J}=11.4 \mathrm{~Hz}, 1 \mathrm{H})$, 3.16-3.13 (m, 2H), 2.93-2.90 (m, 2H), 2.74-2.72 (m, 2H), 2.37-2.35 (m, 4H), 1.74-1.61 (m, $10 \mathrm{H}), 1.50-1.45(\mathrm{~m}, 2 \mathrm{H}), 1.43(\mathrm{~s}, 3 \mathrm{H}), 1.43(\mathrm{~s}, 3 \mathrm{H})$.

${ }^{13} \mathrm{C}$ NMR (150 MHz, $\left.\mathrm{CDCl}_{3}\right): \delta=173.79,173.33,173.31,163.77,163.72,162.76,86.56$, $78.46,71.12$, 68.47, 62.09, 62.07, 60.45, 60.24, 55.54, 55.52, 55.47, 40.68, 40.65, 34.07, $33.86,33.82,28.48,28.45,28.37,24.92,24.81,24.78,23.16,14.39$.

HRMS (ESI-TOF) m/z: calc'd for $\mathrm{C}_{16} \mathrm{H}_{22} \mathrm{Cl}_{3} \mathrm{~N}_{3} \mathrm{O}_{4} \mathrm{~S}$ [M+Na]+ 480.0294 , found 480.0302 .

IR (film) cm': 3271, 3054, 2932, 2863, 2357, 1733, 1695, 1667, 1147, 1089, 1017, 731.

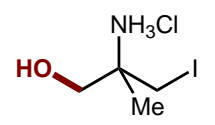

\section{2-(chloro- $\lambda^{5}$-azaneyl)-3-iodo-2-methylpropan-1-ol (48)}

To a 2-dram vial equipped with a PTFE septum cap and magnetic stir bar was added oxazoline $17(68.5 \mathrm{mg}, 0.2 \mathrm{mmol})$ and $\mathrm{MeOH}(1 \mathrm{~mL}) .2 \mathrm{M} \mathrm{HCl}(\mathrm{aq})(0.2 \mathrm{~mL}, 0.4 \mathrm{mmol})$ was added to the vial, and the reaction was stirred at room temperature for $16 \mathrm{~h}$. The reaction was concentrated in vacuo and rinsed with hexanes to afford product $\mathbf{X}$ (50.3 $\mathrm{mg}, 100 \%)$ as a light yellow solid.

${ }^{1} \mathrm{H}$ NMR (400 MHz, d4-MeOD): $\delta=8.22$ (bs, 3H), 3.69 (dd, J = 22.1, $11.5 \mathrm{~Hz}, 2 \mathrm{H}$ ), 3.50 (dd, $\mathrm{J}=11.9,11.1 \mathrm{~Hz}, 2 \mathrm{H}), 1.41(\mathrm{~s}, 3 \mathrm{H})$.

${ }^{13} \mathrm{C}$ NMR (100 MHz, $\left.\mathrm{d}_{4}-\mathrm{MeOD}\right): \delta=65.77,57.66,21.58,8.76$.

HRMS (ESI-TOF) m/z: calc'd for $\mathrm{C}_{4} \mathrm{H}_{11} \mathrm{CIINO}[\mathrm{M}-\mathrm{Cl}]^{+} 215.9900$, found 215.9891 .

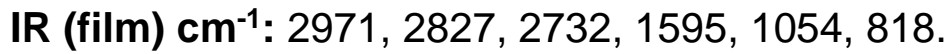


<smiles>NC(CO)(CI)NC(=O)C(Cl)(Cl)Cl</smiles>

\section{2,2,2-trichloro-N-(1-hydroxy-3-iodo-2-methylpropan-2-yl)acetamide (49)}

To a 2-dram vial equipped with a PTFE septum cap and magnetic stir bar was added oxazoline 17 (68.5 mg, $0.2 \mathrm{mmol}$ ) and TsOH monohydrate (38 mg, $0.2 \mathrm{mmol})$. MeCN (1 $\mathrm{mL})$ and $\mathrm{H}_{2} \mathrm{O}(0.5 \mathrm{~mL})$ were added to the vial, and the reaction was stirred at room temperature for $8 \mathrm{~min}$. Upon completion, the solution was dumped into $\mathrm{H}_{2} \mathrm{O}$ and quenched with $\mathrm{NaHCO}_{3}(\mathrm{aq})$. The aqueous layer was extracted $3 x$ with $\mathrm{CH}_{2} \mathrm{Cl}_{2}(5 \mathrm{~mL})$. The combined organic layer was concentrated in vacuo before purification by flash chromatography (isocratic: $15: 85$, EtOAc : hexanes) to afford product 49 (71.4 mg, 99\%) as a yellow oil.

Rf: 0.33 (40\% EtOAc / hexanes).

${ }^{1} \mathrm{H}$ NMR (600 MHz, CDCl 3$): \delta=7.02(\mathrm{bs}, 1 \mathrm{H}), 3.92(\mathrm{~d}, \mathrm{~J}=11.4 \mathrm{~Hz}, 1 \mathrm{H}), 3.79(\mathrm{~d}, \mathrm{~J}=11.3$ $\mathrm{Hz}, 1 \mathrm{H}), 3.70(\mathrm{~d}, \mathrm{~J}=10.6,1 \mathrm{H}), 3.56(\mathrm{~d}, \mathrm{~J}=10.6 \mathrm{~Hz}, 1 \mathrm{H}), 2.73(\mathrm{bs}, 1 \mathrm{H}), 1.51(\mathrm{~s}, 3 \mathrm{H})$.

${ }^{13} \mathrm{C}$ NMR (100 MHz, $\left.\mathrm{CDCl}_{3}\right): \delta=161.67,92.76,66.85,57.75,22.04,12.20$.

HRMS (ESI-TOF) m/z: calc'd for $\mathrm{C}_{6} \mathrm{H}_{9} \mathrm{Cl}_{3} \mathrm{INO}_{2}[\mathrm{M}+\mathrm{Na}]^{+} 358.8700$, found 358.8721 .

IR (film) cm$^{-1}:$ 3462, 3376, 1697, 1045, 816.

MP: $102-104^{\circ} \mathrm{C}$.

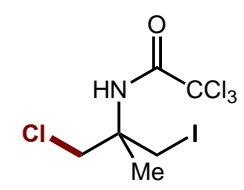

\section{2,2,2-trichloro-N-(1-chloro-3-iodo-2-methylpropan-2-yl)acetamide (50)}

To a 2-dram vial equipped with a PTFE septum cap and magnetic stir bar was added oxazoline $17(68.5 \mathrm{mg}, 0.2 \mathrm{mmol})$ and $\mathrm{CH}_{2} \mathrm{Cl}_{2}(1.3 \mathrm{~mL}) .4 \mathrm{M} \mathrm{HCl}$ in dioxane $(0.2 \mathrm{~mL}, 0.8$ $\mathrm{mmol}$ ) was added to the vial, and the reaction was stirred at room temperature for $12 \mathrm{~h}$. The reaction was quenched with saturated $\mathrm{NaHCO}_{3}$ (aq). The aqueous layer was extracted $3 x$ with $\mathrm{CH}_{2} \mathrm{Cl}_{2}(5 \mathrm{~mL})$. The combined organic layer was concentrated in vacuo before purification by flash chromatography (isocratic: $10: 90$, EtOAc : hexanes) to afford product $50(74.0 \mathrm{mg}, 98 \%)$ as a white solid.

Rf: 0.40 (10\% EtOAc / hexanes). 
${ }^{1} \mathrm{H}$ NMR (400 MHz, $\left.\mathrm{CDCl}_{3}\right): \delta=6.74(\mathrm{bs}, 1 \mathrm{H}), 4.08(\mathrm{~d}, \mathrm{~J}=11.4,1 \mathrm{H}), 3.84(\mathrm{~d}, \mathrm{~J}=10.6$ $\mathrm{Hz}, 1 \mathrm{H}), 3.78(\mathrm{~d}, \mathrm{~J}=11.4 \mathrm{~Hz}, 1 \mathrm{H}), 3.54(\mathrm{~d}, \mathrm{~J}=10.6 \mathrm{~Hz}, 1 \mathrm{H}), 1.63(\mathrm{~s}, 3 \mathrm{H})$.

${ }^{13} \mathrm{C}$ NMR (100 MHz, $\left.\mathrm{CDCl}_{3}\right): \delta=161.0,92.5,57.1,47.8,22.8,11.7$.

HRMS (ESI-TOF) m/z: calc'd for $\mathrm{C}_{6} \mathrm{H}_{8} \mathrm{Cl}_{4} \mathrm{INO}[\mathrm{M}+\mathrm{Na}]^{+} 399.8302$, found 399.8302 .

IR (film) cm$^{-1}:$ 3289, 1693, 1528, 844, 820.

MP: $81-82^{\circ} \mathrm{C}$.

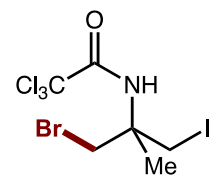

\section{2,2,2-trichloro-N-(1-bromo-3-iodo-2-methylpropan-2-yl)acetamide (51)}

To a 2-dram vial equipped with a PTFE septum cap and magnetic stir bar was added oxazoline 17 (68.5 mg, $0.2 \mathrm{mmol})$ and $\mathrm{CH}_{2} \mathrm{Cl}_{2}(0.5 \mathrm{~mL})$. TMSBr $(52 \mu \mathrm{L}, 0.4 \mathrm{mmol})$ was added to the vial, and the reaction was stirred at room temperature for $30 \mathrm{~min}$. The reaction was concentrated in vacuo before purification by flash chromatography (isocratic: 10 : 90, EtOAc : hexanes) to afford product 51 (78.7 mg, 93\%) as a white solid.

Rf: 0.50 (15\% EtOAc / hexanes).

${ }^{1} \mathrm{H}$ NMR $\left(600 \mathrm{MHz}, \mathrm{CDCl}_{3}\right): \delta=6.74(\mathrm{bs}, 1 \mathrm{H}), 4.03(\mathrm{~d}, \mathrm{~J}=10.7 \mathrm{~Hz}, 1 \mathrm{H}), 3.91(\mathrm{~d}, \mathrm{~J}=10.6$ $\mathrm{Hz}, 1 \mathrm{H}), 3.69(\mathrm{~d}, \mathrm{~J}=10.7 \mathrm{~Hz}, 1 \mathrm{H}), 3.57(\mathrm{~d}, \mathrm{~J}=10.6 \mathrm{~Hz}, 1 \mathrm{H}), 1.67(\mathrm{~s}, 3 \mathrm{H})$.

${ }^{13} \mathrm{C}$ NMR (150 MHz, $\left.\mathrm{CDCl}_{3}\right): \delta=161.05,92.51,56.31,36.88,23.74,11.92$.

HRMS (ESI-TOF) m/z: calc'd for $\mathrm{C}_{6} \mathrm{H}_{8} \mathrm{BrCl}_{3} \mathrm{INO}[\mathrm{M}+\mathrm{Na}]^{+}$443.7797, found 443.7775 .

IR (film) cm$^{-1}:$ 3293, 2981, 2924, 1692, 1528, 1256, 819.

MP: $99-101^{\circ} \mathrm{C}$. 


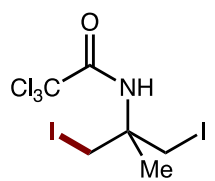

\section{2,2,2-trichloro-N-(1,3-diiodo-2-methylpropan-2-yl)acetamide (51A)}

To a 2-dram vial equipped with PTFE septa cap and magnetic stir bar, was added hexamethyldisilane $(0.4 \mathrm{mmol})$ and $\mathrm{I}_{2}(0.4 \mathrm{mmol})$ under $\mathrm{N}_{2}$. The reaction was refluxed for 1 hour. After cooling to room temperature, oxazoline $17(68.5 \mathrm{mg}, 0.2 \mathrm{mmol})$ was added as a solution in $\mathrm{CH}_{2} \mathrm{Cl}_{2}(1 \mathrm{~mL})$ and stirred for 12 hours. The reaction was concentrated in vacuo before purification by flash chromatography (isocratic: $10: 90$, EtOAc : hexanes) to afford product $\mathbf{X}(50.8 \mathrm{mg}, 54 \%)$ as a white solid.

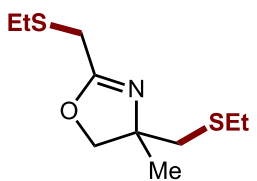

\section{2,4-bis((ethylthio)methyl)-4-methyl-4,5-dihydrooxazole (52)}

To a 2-dram vial equipped with a PTFE septum cap and magnetic stir bar was added oxazoline $17(40 \mathrm{mg}, 0.117 \mathrm{mmol})$ and $\mathrm{K}_{2} \mathrm{CO}_{3}(80.8 \mathrm{mg}, 0.585 \mathrm{mmol})$. The vial was evacuated and refilled with $\mathrm{N}_{2}(3 \mathrm{x})$. Ethanethiol $(0.042 \mathrm{~mL}, 0.585 \mathrm{mmol})$ and DMF (1.67 $\mathrm{mL}$ ) were added to the vial, and the reaction was stirred under $\mathrm{N}_{2}$ at $100^{\circ} \mathrm{C}$ for $48 \mathrm{~h}$. Upon completion, the solution was cooled to room temperature, then diluted with $\mathrm{H}_{2} \mathrm{O}$ and extracted with $\mathrm{CH}_{2} \mathrm{Cl}_{2}(3 \mathrm{x})$. The aqueous layer was extracted $(3 \mathrm{x})$ with $\mathrm{CH}_{2} \mathrm{Cl}_{2}(5 \mathrm{~mL})$. The organic layer was washed with $\mathrm{H}_{2} \mathrm{O}, 1 \mathrm{M} \mathrm{NaOH}$, and brine, then dried over $\mathrm{MgSO}_{4}$. The combined organic layer was concentrated in vacuo before purification by flash chromatography (15\% EtOAc/hexanes) to afford product $52(24.9 \mathrm{mg}, 91 \%)$ as a colorless oil.

Rf: 0.15 (15\% EtOAc/hexanes)

${ }^{1} \mathrm{H}$ NMR $\left(400 \mathrm{MHz}, \mathrm{CDCl}_{3}\right): \delta=4.28(\mathrm{~d}, \mathrm{~J}=8.4 \mathrm{~Hz}, 1 \mathrm{H}), 3.95(\mathrm{~d}, \mathrm{~J}=8.4 \mathrm{~Hz}, 1 \mathrm{H}), 3.27(\mathrm{~s}$, $2 \mathrm{H}), 2.71(\mathrm{~s}, 2 \mathrm{H}), 2.66(\mathrm{q}, \mathrm{J}=7.4 \mathrm{~Hz}, 2 \mathrm{H}), 2.58(\mathrm{q}, \mathrm{J}=7.5,2 \mathrm{H}), 1.35(\mathrm{~s}, 3 \mathrm{H}), 1.29-1.21$ $(\mathrm{m}, 6 \mathrm{H})$.

${ }^{13} \mathrm{C}$ NMR $\left(100 \mathrm{MHz}, \mathrm{CDCl}_{3}\right): \delta=164.27,77.35,71.46,42.79,27.95,27.92,26.85,26.73$, $15.02,14.42$.

HRMS (ESI-TOF) m/z: calc'd for $\mathrm{C}_{10} \mathrm{H}_{19} \mathrm{NOS}_{2}[\mathrm{M}+\mathrm{Na}]^{+} 256.0800$, found 256.0796.

IR (film) cm': 2965, 2925, 2870, 1666, 1451, 1409, 1374, 1267, 730. 


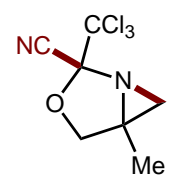

\section{5-methyl-2-(trichloromethyl)-3-oxa-1-azabicyclo[3.1.0]hexane-2-carbonitrile (53)}

To a 2-dram vial equipped with a PTFE septum cap and magnetic stir bar was added oxazoline 17 (68.5 mg, $0.2 \mathrm{mmol})$ and $\mathrm{NaCN}(49.0 \mathrm{mg}, 1.0 \mathrm{mmol})$. Dry DMF (1 mL) was added to the vial, and the reaction was stirred at $50^{\circ} \mathrm{C}$ for $12 \mathrm{~h}$. Upon completion, the solution was cooled to room temperature then dumped into aqueous $\mathrm{NaHCO}_{3}(5 \mathrm{~mL})$ and $\mathrm{Et}_{2} \mathrm{O}(5 \mathrm{~mL})$. The aqueous layer was extracted $3 x$ with EtOAc $(5 \mathrm{~mL})$. The organic layer was washed $5 x$ with $\mathrm{H}_{2} \mathrm{O}(5 \mathrm{~mL})$. The combined organic layer was concentrated in vacuo before purification by flash chromatography (isocratic: $20: 80$, EtOAc : hexanes) to afford product $53(40.1 \mathrm{mg}, 83 \%, 8: 1)$ as a colorless oil.

Rf: 0.35 (20\% EtOAc / hexanes).

${ }^{1} \mathrm{H}$ NMR $\left(400 \mathrm{MHz}, \mathrm{CDCl}_{3}\right): \delta=4.41(\mathrm{~d}, \mathrm{~J}=8.2 \mathrm{~Hz}, 1 \mathrm{H}), 4.20(\mathrm{dd}, \mathrm{J}=8.1,1.7 \mathrm{~Hz}, 1 \mathrm{H})$, $2.35(\mathrm{~d}, 1.3 \mathrm{~Hz}, 1 \mathrm{H}), 2.14(\mathrm{t}, 1.4 \mathrm{~Hz}, 1 \mathrm{H}), 1.51(\mathrm{~s}, 3 \mathrm{H})$.

${ }^{13} \mathrm{C}$ NMR (175 MHz, $\left.\mathrm{CDCl}_{3}\right): \delta=113.79,104.73,75.74,52.28,38.46,29.84,16.96$.

HRMS (ESI-TOF) m/z: calc'd for $\mathrm{C}_{7} \mathrm{H}_{7} \mathrm{Cl}_{3} \mathrm{~N}_{2} \mathrm{O}[\mathrm{M}+\mathrm{Na}]^{+}$262.9522, found 262.9521.

IR (film) cm-1: 2973, 2906, 2357, 1177, 1021, 839, 819.

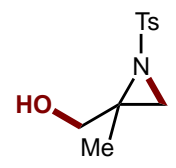

\section{(2-methyl-1-tosylaziridin-2-yl)methanol (54)}

To a 2-dram vial equipped with a PTFE septum cap and magnetic stir bar was added oxazoline $17(68.5 \mathrm{mg}, 0.2 \mathrm{mmol})$ and $6 \mathrm{M} \mathrm{HCl}(0.3 \mathrm{~mL})$. $\mathrm{MeOH}(1 \mathrm{~mL})$ was added to the vial, and the reaction was stirred at room temperature until starting material was consumed as determined by TLC (1h). The solution was concentrated in vacuo, and the residue was dissolved in $\mathrm{MeOH}(1 \mathrm{~mL})$ and excess $\mathrm{KHCO}_{3}$ was added. The suspension was stirred at room temperature for 45 mins before being concentrated under reduced pressure. The residue was dissolved in $\mathrm{CH}_{2} \mathrm{Cl}_{2}(1 \mathrm{~mL})$ and cooled to $0^{\circ} \mathrm{C}$ followed by addition of $\mathrm{TsCl}(46 \mathrm{mg}, 0.24 \mathrm{mmol})$ and $\mathrm{Et}_{3} \mathrm{~N}(34 \mu \mathrm{L}, 0.24 \mathrm{mmol})$. The solution was stirred for $1.5 \mathrm{hr}$ before being dumped into $\mathrm{H}_{2} \mathrm{O}$. The aqueous layer was extracted $3 x$ with $\mathrm{CH}_{2} \mathrm{Cl}_{2}$ $(5 \mathrm{~mL})$. The combined organic layer was concentrated in vacuo before purification by 
flash chromatography (isocratic: $50: 50$, EtOAc : hexanes) to afford product 54 (29.4 mg, $61 \%)$ as an off-white solid.

Rf: 0.26 (40\% EtOAc / hexanes).

${ }^{1} \mathrm{H}$ NMR (400 MHz, $\left.\mathrm{CDCl}_{3}\right): \delta=7.82(\mathrm{~d}, \mathrm{~J}=8.3,2 \mathrm{H}), 7.33(\mathrm{~d}, \mathrm{~J}=8.5,2 \mathrm{H}), 3.85(\mathrm{dd}, \mathrm{J}=$ 12.6, $6.9 \mathrm{~Hz}, 1 \mathrm{H}), 3.70(\mathrm{dd}, \mathrm{J}=12.7,6.9 \mathrm{~Hz}, 1 \mathrm{H}), 2.62(\mathrm{~s}, 1 \mathrm{H}), 2.53(\mathrm{~s}, 1 \mathrm{H}), 2.44(\mathrm{~s}, 3 \mathrm{H})$, $2.33(\mathrm{t}, \mathrm{J}=7.0,1 \mathrm{H}), 1.64(\mathrm{~s}, 3 \mathrm{H})$.

${ }^{13} \mathrm{C}$ NMR (100 MHz, $\left.\mathrm{CDCl}_{3}\right): \delta=144.41,137.60,129.83,127.51,65.33,51.78,38.83$, 21.80, 17.38.

HRMS (ESI-TOF) m/z: calc'd for $\mathrm{C}_{11} \mathrm{H}_{15} \mathrm{NO}_{3} \mathrm{~S}[\mathrm{M}+\mathrm{Na}]^{+} 264.0700$, found 264.0669.

IR (film) cm$^{-1}:$ 3295, 3035, 1693, 1382, 1157.

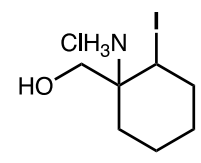

\section{(1-amino-2-iodocyclohexyl)methanol chloride salt (56)}

To a 2-dram vial equipped with a PTFE septum cap and magnetic stir bar was added oxazoline 2 (360 mg, $0.94 \mathrm{mmol})$ and $\mathrm{MeOH}(5 \mathrm{~mL})$. $2 \mathrm{M} \mathrm{HCl}(\mathrm{aq})(1.0 \mathrm{~mL}, 1.9 \mathrm{mmol})$ was added to the vial, and the reaction was stirred at room temperature for $16 \mathrm{~h}$. The reaction was concentrated in vacuo and rinsed with hexanes to afford product $56(209.2 \mathrm{mg}$, $100 \%$ ) as a white solid. A single crystal was prepared using methanol and ether through vapor diffusion at room temperature.

${ }^{1} \mathrm{H}$ NMR (400 MHz, d4-MeOD): $\delta=4.42(\mathrm{dd}, \mathrm{J}=11.4,4.4 \mathrm{~Hz}), 3.95(\mathrm{~d}, \mathrm{~J}=11.7 \mathrm{~Hz}), 3.78$ $(\mathrm{dd}, \mathrm{J}=11.7,1.1 \mathrm{~Hz}), 2.33-2.25(\mathrm{~m}, 2 \mathrm{H}), 2.11-2.04(\mathrm{~m}, 1 \mathrm{H}), 1.77-1.70(\mathrm{~m}, 1 \mathrm{H}), 1.63-1.44$ $(\mathrm{m}, 4 \mathrm{H})$.

${ }^{13} \mathrm{C}$ NMR (100 MHz, $\left.\mathrm{d}_{4}-\mathrm{MeOD}\right): \delta=63.98,60.96,37.48,34.12,31.30,28.27,21.93$.

HRMS (ESI-TOF) m/z: calc'd for $\mathrm{C}_{4} \mathrm{H}_{11} \mathrm{CIINO}[\mathrm{M}-\mathrm{Cl}]^{+} 256.0198$, found 256.0170 .

IR (film) cm-1: 3222, 3070, 2980, 2883, 1600, 1524, 1062.

MP: $191-193^{\circ} \mathrm{C}$. 
VIII. One-pot Procedure
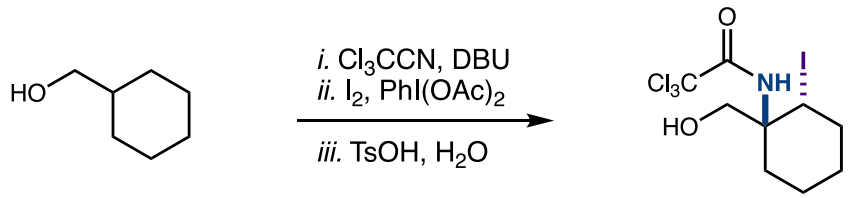

\section{2,2,2-trichloro-N-(1-(hydroxymethyl)-2-iodocyclohexyl)acetamide (55)}

Cyclohexylmethanol $(2.15 \mathrm{~mL}, 0.2 \mathrm{mmol})$ was subjected to GP1. Upon completion (monitored by TLC) the reaction was concentrated and subjected to GP2. Upon completion, the reaction was concentrated. $\mathrm{TsOH}$ monohydrate $(38 \mathrm{mg}, 0.2 \mathrm{mmol}$ ), $\mathrm{MeCN}(1 \mathrm{~mL})$ and $\mathrm{H}_{2} \mathrm{O}(0.5 \mathrm{~mL})$ were added to the vial, and the reaction was stirred at room temperature. Upon completion (as determined by TLC), the solution was dumped into $\mathrm{H}_{2} \mathrm{O}$ and quenched with $\mathrm{NaHCO}_{3}(\mathrm{aq})$. The aqueous layer was extracted $3 \mathrm{x}$ with $\mathrm{CH}_{2} \mathrm{Cl}_{2}(5 \mathrm{~mL})$. The combined organic layer was concentrated in vacuo before purification by flash chromatography (isocratic: $15: 85$, EtOAc : hexanes) to afford product 55 (57.7 $\mathrm{mg}, 72 \%$ ) as a pale yellow solid.

Rf: 0.37 (20\% EtOAc / hexanes).

${ }^{1} \mathrm{H}$ NMR $\left(600 \mathrm{MHz}, \mathrm{CDCl}_{3}\right): \delta=7.24(\mathrm{bs}, 1 \mathrm{H}), 5.09(\mathrm{dd}, \mathrm{J}=10.7,4.3 \mathrm{~Hz}, 1 \mathrm{H}), 4.09(\mathrm{~d}, \mathrm{~J}$ $=12.0 \mathrm{~Hz}, 1 \mathrm{H}), 3.94(\mathrm{~d}, \mathrm{~J}=11.9 \mathrm{~Hz}, 1 \mathrm{H}), 2.68(\mathrm{bs}, 1 \mathrm{H}), 2.36(\mathrm{~m}, 1 \mathrm{H}), 2.22-2.18(\mathrm{~m}, 1 \mathrm{H})$, 2.15-2.09 (m, 2H), 1.72-1.64 (m, 2H), 1.56-1.50 (m, 2H).

${ }^{13} \mathrm{C}$ NMR $\left(100 \mathrm{MHz}, \mathrm{CDCl}_{3}\right): \delta=161.42,93.03,66.16,60.69,35.93,34.61,30.59,26.81$, 21.54.

HRMS (ESI-TOF) m/z: calc'd for $\mathrm{C}_{9} \mathrm{H}_{13} \mathrm{Cl}_{3} \mathrm{INO}_{2}[\mathrm{M}+\mathrm{Na}]^{+} 421.8954$, found 421.8956 .

IR (film) cm$^{-1}:$ 3368, 2939, 2865, 1697, 1510, 818. 


\section{C-H lodination}<smiles>CC1(COC(=N)C(Cl)(Cl)Cl)CCCCC1</smiles>

(1-iodocyclohexyl)methyl 2,2,2-trichloroacetimidate (4)

To a 2-dram vial equipped with a PTFE septum cap and magnetic stir bar, was added imidate 1 (51.7 mg, $0.2 \mathrm{mmol})$, iodobenzene diacetate (77.3 mg. $0.24 \mathrm{mmol}$ ) and Nal (36.0 mg, $0.24 \mathrm{mmol})$. This vial was evacuated and backfilled with $\mathrm{N}_{2}(3 \mathrm{x})$. Dry, degassed ${ }^{\mathrm{t}} \mathrm{BuOH}(0.66 \mathrm{~mL})$ was added to the vial under $\mathrm{N}_{2}$. The reaction was irradiated with two $26 \mathrm{~W}$ compact fluorescent light bulbs and cooled by two fans for $1 \mathrm{~h}$. Upon completion, the solution was quenched via a reductive workup with $20 \% \mathrm{Na}_{2} \mathrm{~S}_{2} \mathrm{O}_{3}$. The aqueous layer was extracted with EtOAc (3x). The combined organic layer was concentrated in vacuo before purification by flash chromatography (1\% EtOAc/hexanes with $\left.1 \% \mathrm{Et}_{3} \mathrm{~N}\right)$ to afford iodinated product $4(57.2 \mathrm{mg}, 74 \%)$ as a yellow oil.

Rf: 0.44 (5\% EtOAc/hexanes)

${ }^{1} \mathrm{H}$ NMR $\left(400 \mathrm{MHz}, \mathrm{CDCl}_{3}\right): \delta=8.36(\mathrm{bs}, 1 \mathrm{H}), 4.60(\mathrm{~s}, 2 \mathrm{H}), 2.14-2.10(\mathrm{~m}, 2 \mathrm{H}), 1.81-$ $1.71(\mathrm{~m}, 6 \mathrm{H}), 1.44-1.37(\mathrm{~m}, 2 \mathrm{H})$.

${ }^{13} \mathrm{C}$ NMR (100 MHz, $\left.\mathrm{CDCl}_{3}\right): \delta=162.41,91.39,79.46,57.58,39.53,25.43,24.25$.

HRMS (ESI-TOF) m/z: calc'd for $\mathrm{C}_{9} \mathrm{H}_{13} \mathrm{Cl}_{3} \mathrm{INO}[\mathrm{M}+\mathrm{H}]^{+} 383.9180$, found 383.9197.

IR (neat) cm$^{-1}:$ 3343, 2929, 2857, 1663, 1446, 1301, 1284, 1076, 792. 


\section{Rate Experiments}

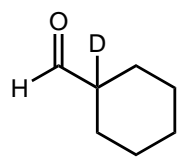

\section{cyclohexane-1- $d$-1-carbaldehyde}

To a $10 \mathrm{~mL}$ pressure tube equipped with a magnetic stir bar was added cyclohexane carbaldehyde $(610 \mu \mathrm{L}, 5 \mathrm{mmol})$ and $\mathrm{D}_{2} \mathrm{O}$. The reaction was heated to $100^{\circ} \mathrm{C}$ and allowed to stir overnight. After cooling to room temperature, $1 \mathrm{M} \mathrm{HCl}(3 \mathrm{~mL})$ and $\mathrm{CH}_{2} \mathrm{Cl}_{2}(12 \mathrm{~mL})$ were added. The aqueous layer was extracted $3 x$ with $\mathrm{CH}_{2} \mathrm{Cl}_{2}(10 \mathrm{~mL})$. The combined organic layer was washed with $\mathrm{NaHCO}_{3}$ (sat. aq) and brine before being dried on anhydrous $\mathrm{MgSO}_{4}$. The organic layer was concentrated in vacuo. ${ }^{1} \mathrm{H}$-NMR analysis indicated a deuterium incorporation of $75 \%$.

The crude mixture was subjected to the above procedure one additional time to afford the title compound with $98 \%$ deuterium incorporation which was carried forward without any additional purification.

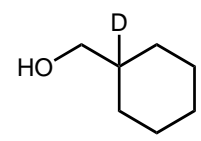

\section{(cyclohexyl-1-d)methanol}

To a $10 \mathrm{~mL}$ round bottom flask equipped with a magnetic stir bar was added aldehyde (226 mg, $2.0 \mathrm{mmol})$ and $d_{4}-\mathrm{MeOD}(2.0 \mathrm{~mL})$. The mixture was brought to $0^{\circ} \mathrm{C}$, and $\mathrm{NaBH}_{4}$ (151 mg, $4.0 \mathrm{mmol}$ ) was added portionwise. The solution was brought to room temperature and allowed to stir $1 \mathrm{~h}$. After starting material had been consumed, the reaction was quenched with brine. The aqueous layer was extracted $3 x$ with $\mathrm{CH}_{2} \mathrm{Cl}_{2}(10$ $\mathrm{mL}$ ), and the combine organic layer was dried over anhydrous $\mathrm{MgSO}_{4}$ and concentrated in vacuo to yield the title compound as a colorless oil. ${ }^{1} \mathrm{H}$-NMR analysis indicated a deuterium incorporation of $>95 \%$. Spectroscopic data matches literature reports.

${ }^{1} \mathrm{H}$ NMR $\left(400 \mathrm{MHz}, \mathrm{CDCl}_{3}\right): \delta=3.31(\mathrm{~s}, 2 \mathrm{H}), 1.75-1.64(\mathrm{~m}, 5 \mathrm{H}), 1.30-1.14(\mathrm{~m}, 3 \mathrm{H})$, $0.930 .87(\mathrm{~m}, 2 \mathrm{H})$.

${ }^{13} \mathrm{C}$ NMR (100 MHz, $\left.\mathrm{CDCl}_{3}\right): \delta=68.79,41.18(\mathrm{t}, \mathrm{J}=19.1 \mathrm{~Hz}), 30.74,27.77,27.02$.

Note: If $\mathrm{MeOH}$ was used in lieu of $d_{4}-\mathrm{MeOD}$, erosion of deuterium incorporation was observed. 


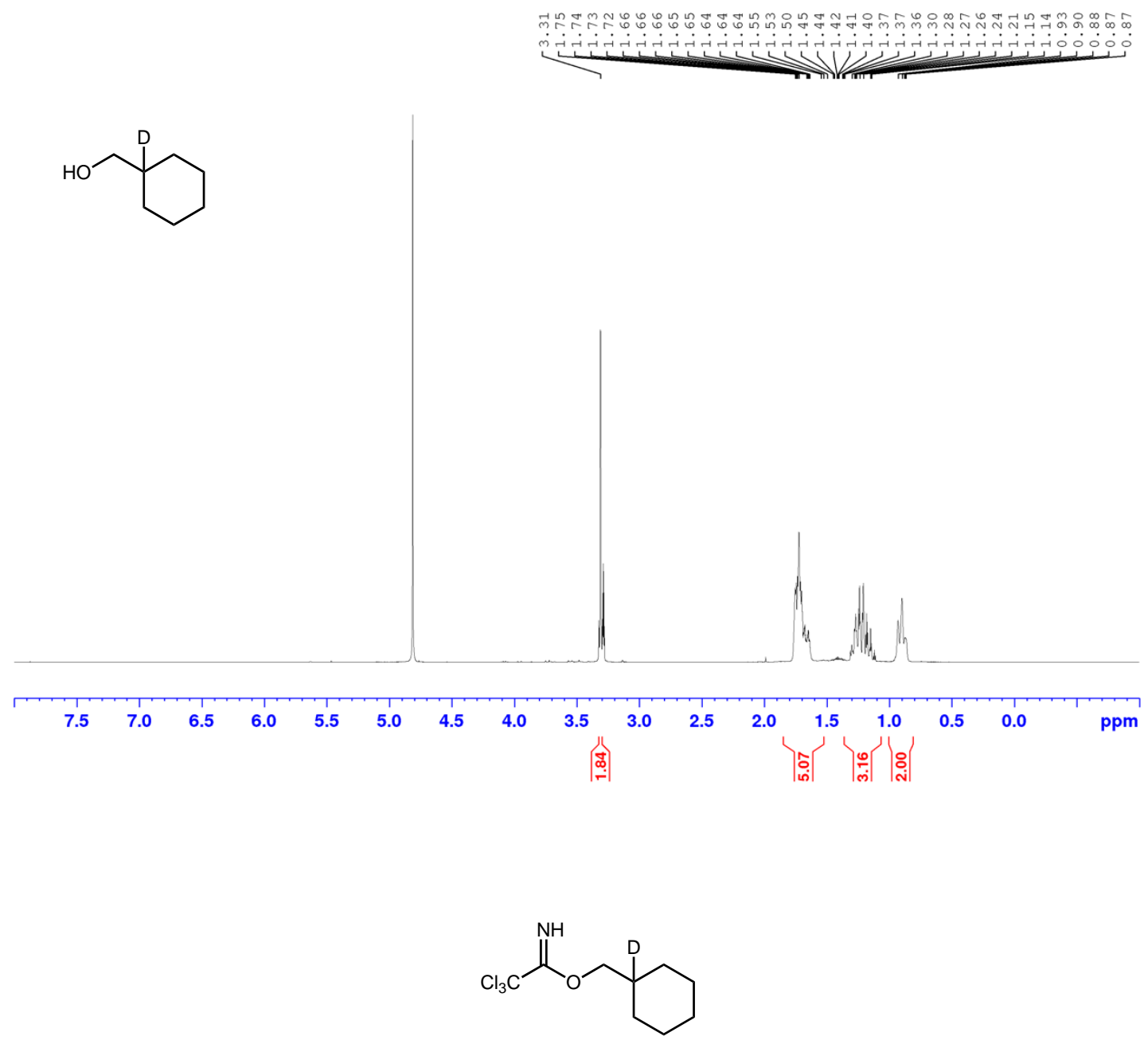

(cyclohexyl-1-d)methyl 2,2,2-trichloroacetimidate (1-D)

Alcohol (461 mg, $5 \mathrm{mmol}$ ) was subjected to GP1. Upon completion (monitored by TLC) the reaction was concentrated and loaded directly onto silica gel and purified by flash column chromatography (isocratic: $1: 98: 1$, EtOAc : hexanes : $\mathrm{Et}_{3} \mathrm{~N}$ ) to yield imidate 1 $D(1.3 \mathrm{~g}, 100 \%)$ as a colorless oil.

Rf: 0.47 (10\% EtOAc / hexanes).

${ }^{1} \mathrm{H}$ NMR (400 MHz, $\left.\mathrm{CDCl}_{3}\right): \delta=8.20(\mathrm{bs}, 1 \mathrm{H}), 4.08(\mathrm{~s}, 2 \mathrm{H}), 1.83-1.79(\mathrm{~m}, 2 \mathrm{H}), 1.77-1.67$ $(\mathrm{m}, 3 \mathrm{H}), 1.37-1.17(\mathrm{~m}, 3 \mathrm{H}), 1.08-1.02(\mathrm{~m}, 2 \mathrm{H})$.

${ }^{13} \mathrm{C}$ NMR (100 MHz, $\left.\mathrm{CDCl}_{3}\right): \delta=163.27,91.85,74.67,36.57(\mathrm{t}, \mathrm{J}=19.3 \mathrm{~Hz}), 29.54$, $26.48,25.78$. 
HRMS (ESI-TOF) m/z: calc'd for $\mathrm{C}_{9} \mathrm{H}_{13} \mathrm{DCl}_{3} \mathrm{NO}[\mathrm{M}+\mathrm{H}]^{+}$259.0282, found 259.0277 .

IR (film) cm': 3345, 2923, 2852, 1662, 1078, 793.

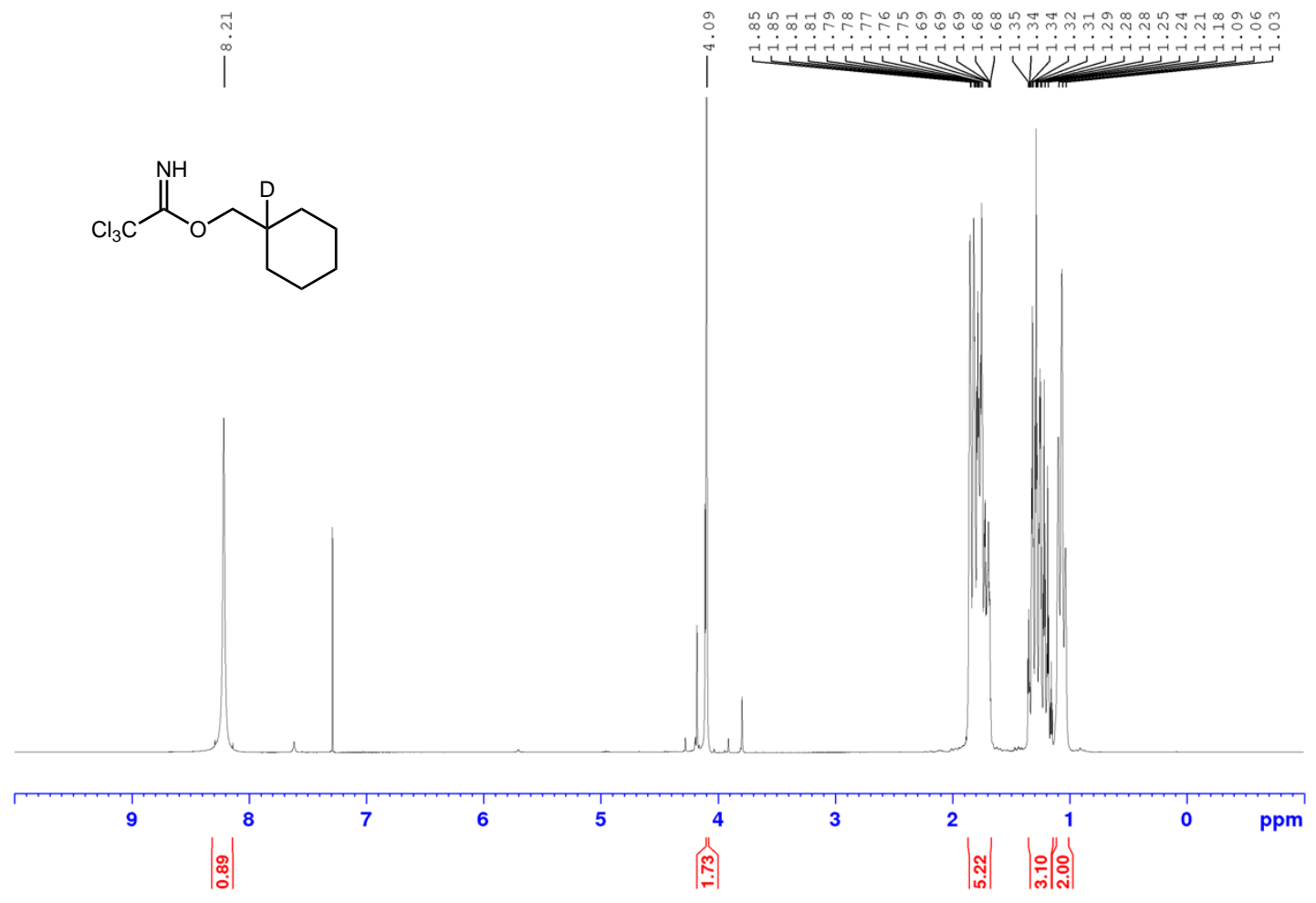


Intramolecular KIE (HAT)

Initial rate for $\mathbf{C}-\mathrm{H}$ iodination<smiles>N=C(OCC1(O)CCCCC1)C(Cl)(Cl)Cl</smiles><smiles></smiles>

Imidate 1 (51.7 mg, $0.2 \mathrm{mmol}$ ) was subjected to GP4. The ${ }^{1} \mathrm{H}-\mathrm{NMR}$ of each sample was collected and analyzed for formation of alkyl iodide intermediate.

Analysis: The NMR yields of alkyl iodide 4 were plotted against time in seconds. The slope was utilized to calculate the initial rate of the reaction. The procedure was repeated, and the average of two trials was taken. Calculated values are tabulated below.

Initial rates for $\beta-H$ cyclohexylmethanol imidate

$\begin{array}{lll}\text { Run } & \text { H-initial rate } & \mathbf{R}^{2} \\ \mathbf{1} & 0.1777 & 0.998 \\ \mathbf{2} & 0.1897 & 0.994 \\ \text { Avg. } & \mathbf{0 . 1 8 3 7} & \end{array}$

Initial rates for $\beta-D$ cyclohexylmethanol imidate

\begin{tabular}{lll} 
Run & D-initial rate & $\mathbf{R}^{2}$ \\
\hline $\mathbf{1}$ & 0.0877 & 0.999 \\
$\mathbf{2}$ & 0.0743 & 0.979 \\
Avg. & $\mathbf{0 . 0 8 1}$ &
\end{tabular}

$$
\mathrm{KIE}(\mathrm{HAT})=\mathrm{K}_{\mathrm{H}} / \mathrm{K}_{\mathrm{D}}=2.3
$$




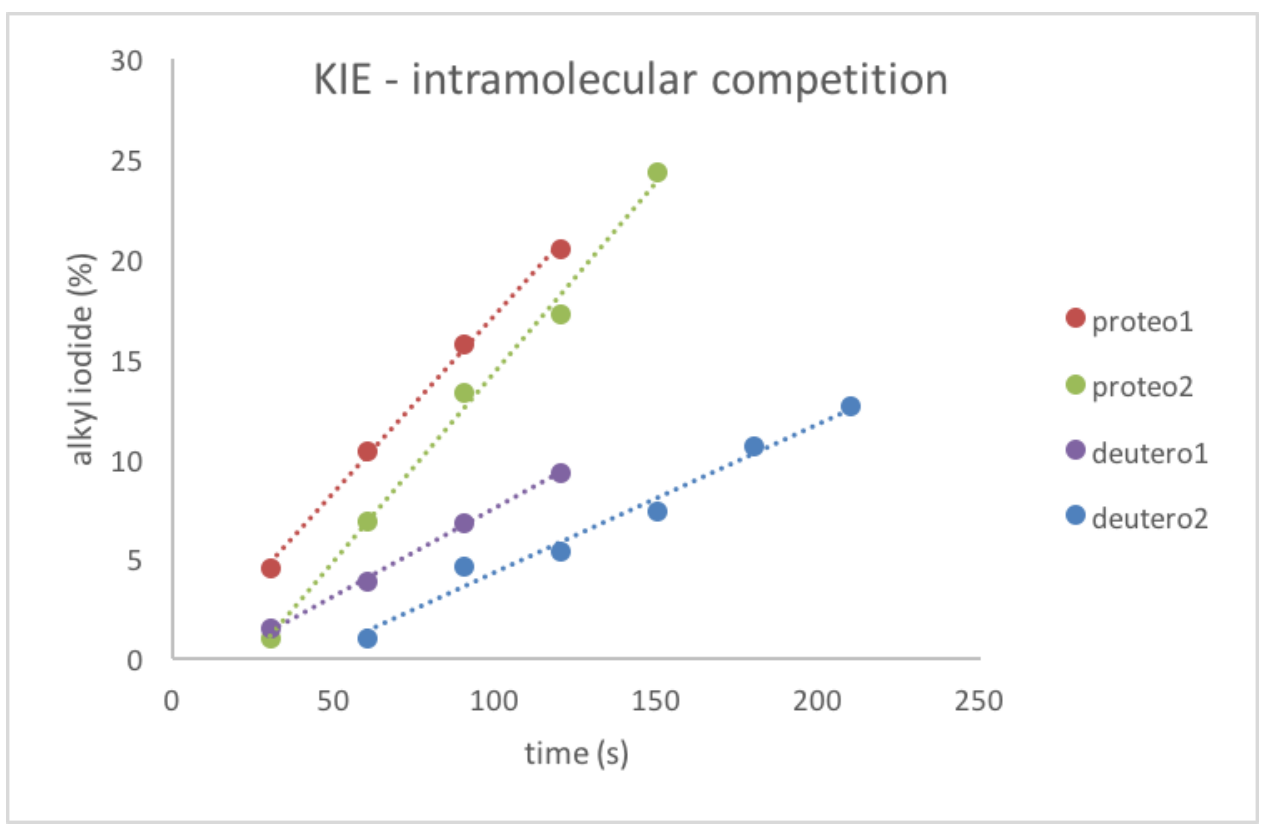

Intramolecular KIE (amino-iodination)

Initial rate for amino-iodination (from imidate 1-D)
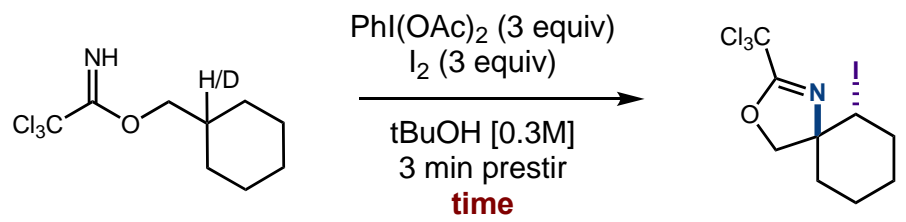

Imidate 1 (51.7 $\mathrm{mg}, 0.2 \mathrm{mmol}$ ) was subjected to GP4. The ${ }^{1} \mathrm{H}-\mathrm{NMR}$ of each sample was collected and analyzed. The ${ }^{1} \mathrm{H}$-NMR of each sample was collected and analyzed for formation of amino-iodinated product.

Analysis: The NMR yields of amino-iodinated product $\mathbf{2}$ were plotted against time in seconds. The slope was utilized to calculate the initial rate of the reaction. The procedure was repeated, and the average of three trials was taken. Calculated values are tabulated below.

Initial rates for $\beta$-H cyclohexylmethanol imidate

\begin{tabular}{|lll|} 
Run & H-initial rate & $\mathbf{R}^{\mathbf{2}}$ \\
\hline $\mathbf{1}$ & 0.0853 & 0.983 \\
$\mathbf{2}$ & 0.0887 & 0.971 \\
\hline Avg. & $\mathbf{0 . 0 8 7 0}$ & \\
\hline
\end{tabular}


Initial rates for $\beta$-D cyclohexylmethanol imidate

$\begin{array}{lll}\text { Run } & \text { D-initial rate } & \mathbf{R}^{\mathbf{2}} \\ \mathbf{1} & 0.0747 & 0.9999 \\ \mathbf{2} & 0.0743 & 0.990 \\ \text { Avg. } & \mathbf{0 . 0 7 4 5} & \end{array}$

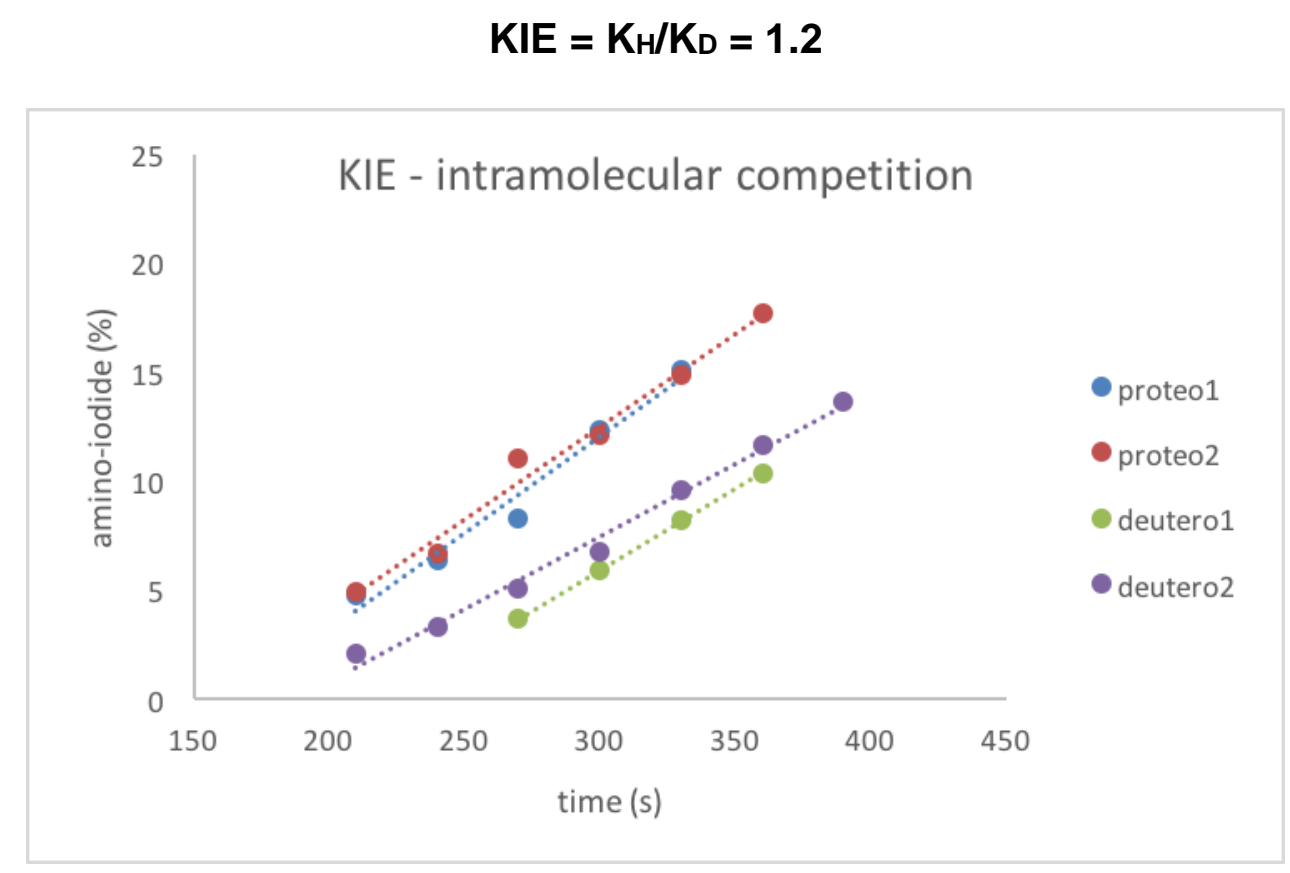

Relative Rate Studies

Initial rate for $\mathrm{C}-\mathrm{H}$ iodination (from imidate 1 )

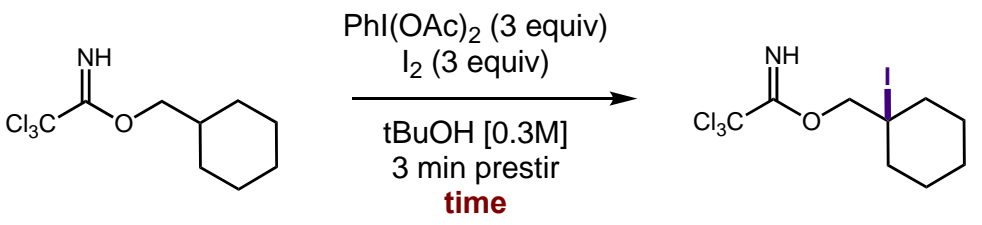

Imidate 1 (51.7 mg, $0.2 \mathrm{mmol})$ was subjected to GP4. The ${ }^{1} \mathrm{H}-\mathrm{NMR}$ of each sample was collected and analyzed. The ${ }^{1} \mathrm{H}$-NMR of each sample was collected and analyzed for formation of alkyl iodide intermediate. 
Analysis: The NMR yields of alkyl iodide 4 were plotted against time in seconds. The slope was utilized to calculate the initial rate of the reaction. The procedure was repeated, and the average of three trials was taken. Calculated values are tabulated below.

Initial rates for cyclohexylmethanol imidate iodination

\begin{tabular}{lll} 
Run & Initial rate & $\mathbf{R}^{\mathbf{2}}$ \\
\hline $\mathbf{1}$ & 0.1777 & 0.998 \\
$\mathbf{2}$ & 0.1897 & 0.994 \\
Avg. & $\mathbf{0 . 1 8 3 7}\left(\mathbf{k}_{1}\right)$ & \\
\hline
\end{tabular}

Initial rate for amino-iodination (from iodo-imidate 4)
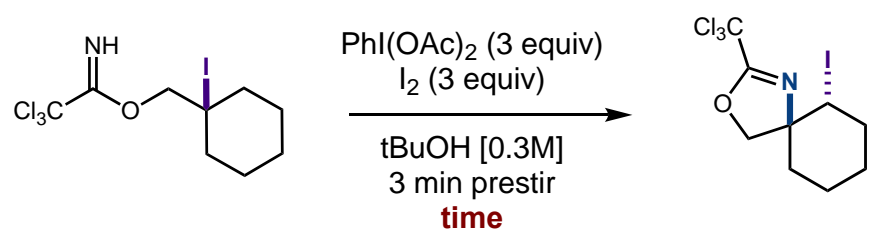

Imidate 4 (51.7 $\mathrm{mg}, 0.2 \mathrm{mmol})$ was subjected to GP4. The ${ }^{1} \mathrm{H}-\mathrm{NMR}$ of each sample was collected and analyzed. The ${ }^{1} \mathrm{H}$-NMR of each sample was collected and analyzed for formation of amino-iodinated product.

Analysis: The NMR yields of amino iodinated product 2 were plotted against time in seconds. The slope was utilized to calculate the initial rate of the reaction. The procedure was repeated, and the average of three trials was taken. Calculated values are tabulated below.

Initial rates for amino-iodination from iodo-imidate

\begin{tabular}{lll} 
Run & Initial rate & $\mathbf{R}^{2}$ \\
\hline $\mathbf{1}$ & 0.1059 & 0.912 \\
$\mathbf{2}$ & 0.1220 & 0.973 \\
$\mathbf{3}$ & 0.1085 & 0.973 \\
Avg. & $\mathbf{0 . 1 1 0 7}\left(\mathbf{k}_{2}\right)$ & \\
\hline
\end{tabular}


Initial rate for amino-iodination (from imidate 1)<smiles>N=C(OCC1CCCCC1)C(Cl)(Cl)Cl</smiles>
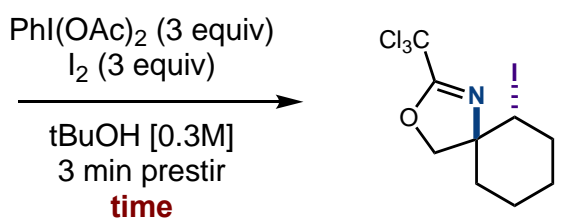

Imidate 1 (51.7 mg, $0.2 \mathrm{mmol}$ ) was subjected to GP4. The ${ }^{1} \mathrm{H}-\mathrm{NMR}$ of each sample was collected and analyzed. The ${ }^{1} \mathrm{H}-\mathrm{NMR}$ of each sample was collected and analyzed for formation of amino-iodinated product.

Analysis: The NMR yields of amino-iodinated product 4 were plotted against time in seconds. The slope was utilized to calculate the initial rate of the reaction. The procedure was repeated, and the average of three trials was taken. Calculated values are tabulated below.

Initial rates for cyclohexylmethanol imidate amino-iodination

\begin{tabular}{lll} 
Run & Initial rate & $\mathbf{R}^{2}$ \\
\hline $\mathbf{1}$ & 0.0853 & 0.983 \\
$\mathbf{2}$ & 0.0887 & 0.971 \\
\hline Avg. & $\mathbf{0 . 0 8 7 0}\left(\mathrm{k}_{3}\right)$ & \\
\hline
\end{tabular}

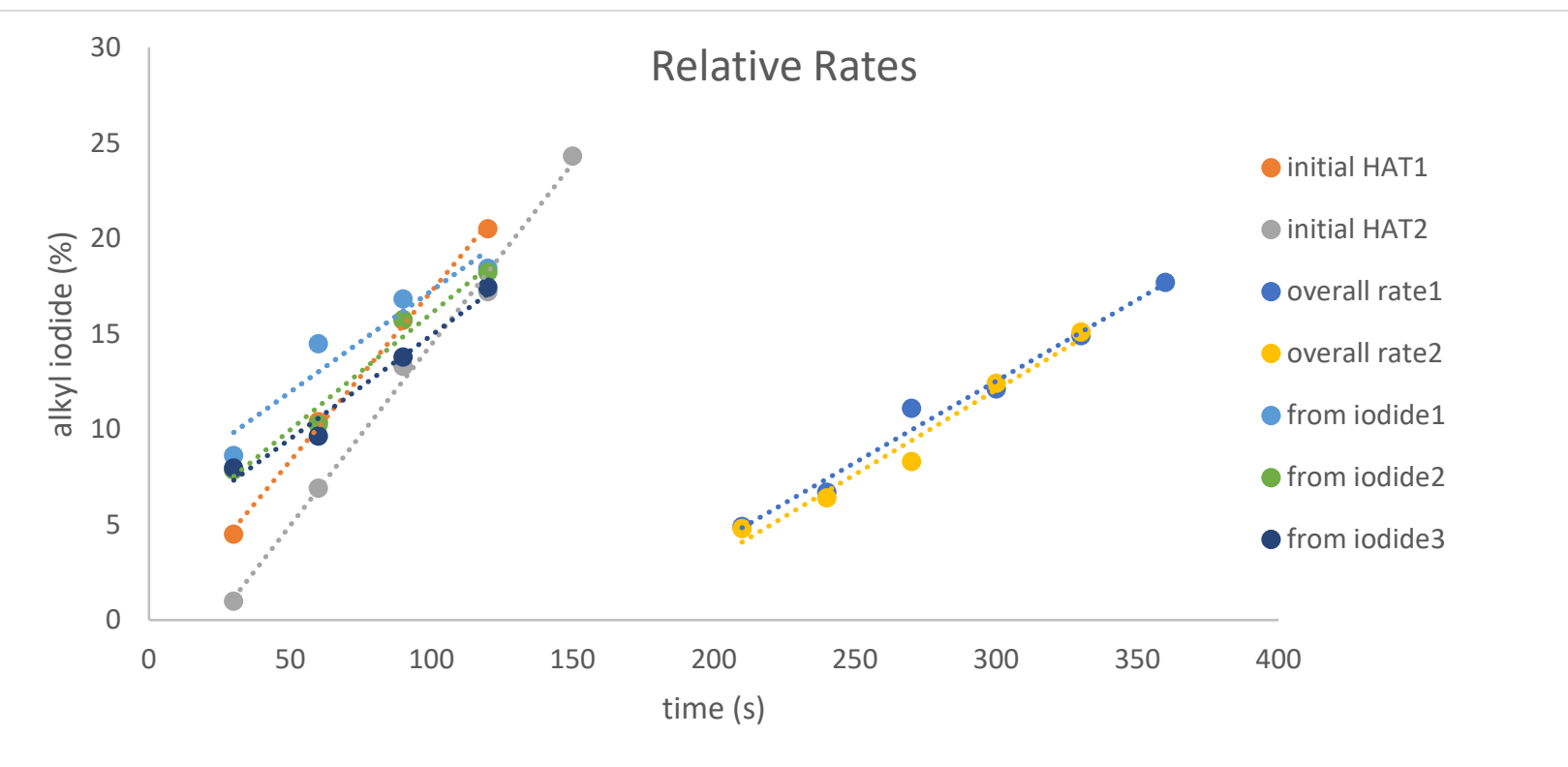

\begin{tabular}{lll}
$\mathbf{k}$ & Initial rate $(\mathbf{a v g})$ & Relative Rate \\
\hline $\mathbf{1}$ & 0.1837 & $\mathbf{2 . 1}$ \\
$\mathbf{2}$ & 0.1107 & 1.3 \\
$\mathbf{3}$ & 0.0870 & 1.0 \\
\hline
\end{tabular}




\section{Mechanistic Experiments and Reaction Profile}

\section{Amino-iodination reaction profile}<smiles>N=C(OCC1CCCCC1)C(Cl)(Cl)Cl</smiles><smiles>N=C(OCC1(I)CCCCC1)C(Cl)(Cl)Cl</smiles><smiles>ClC1=NC2(CCCCC2I)CO1</smiles><smiles>ClC(Cl)(Cl)C1=NC2(CCCCC2)CO1</smiles>

Imidate 1 (51.7 mg, $0.2 \mathrm{mmol}$ ) was subjected to GP4. The ${ }^{1} \mathrm{H}-\mathrm{NMR}$ of each sample was collected and analyzed. The ${ }^{1} \mathrm{H}$-NMR of each sample was collected and analyzed for product distribution.

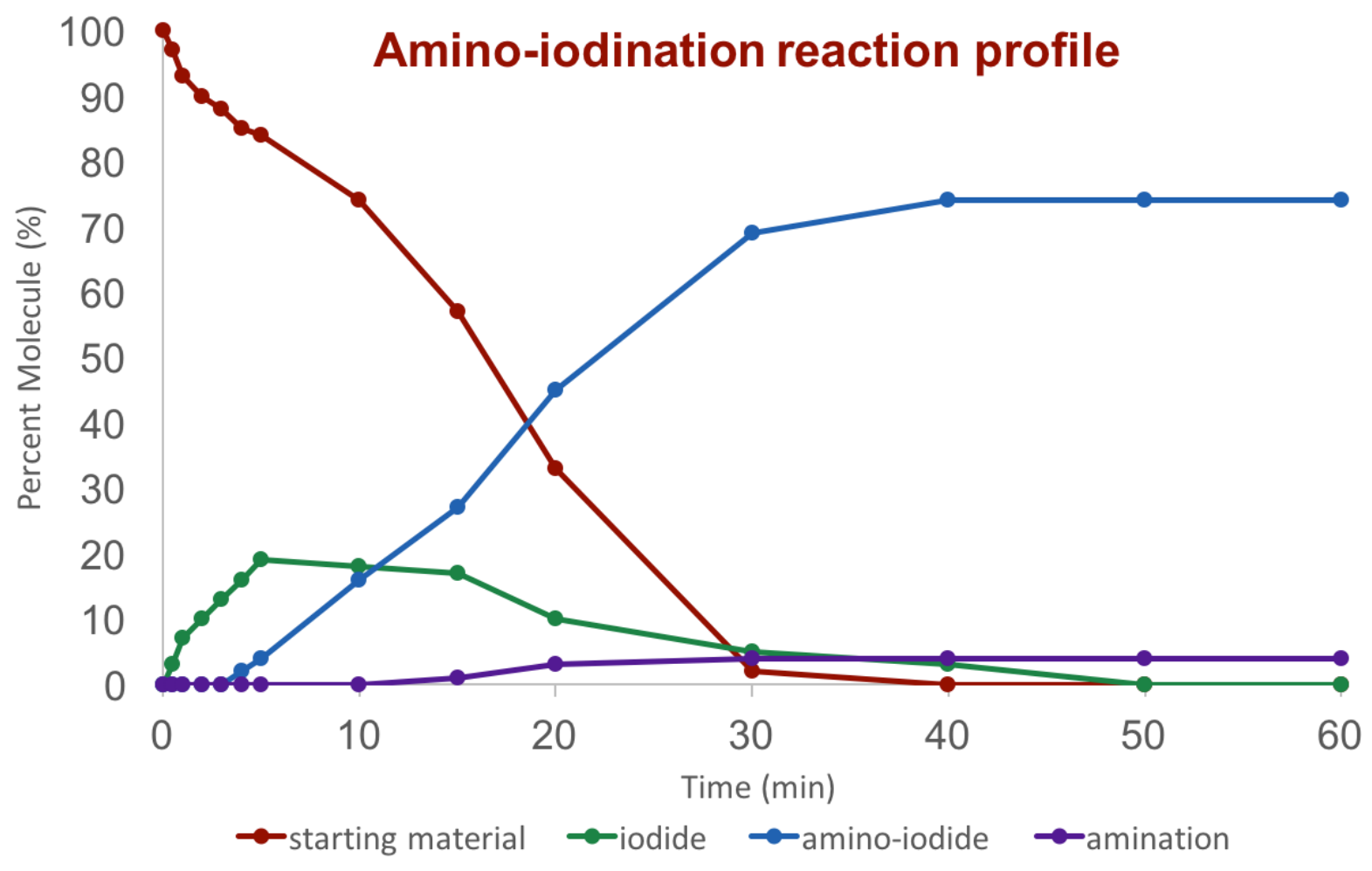


<smiles>CC(=O)OCC1CCCCC1</smiles>

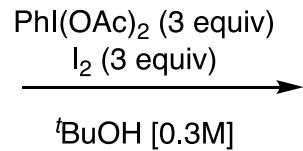<smiles>CC(=O)OCC1(C)CCCCC1</smiles>

not observed

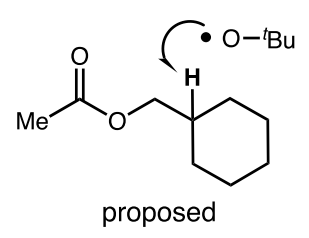

Cyclohexylmethyl acetate was subjected to GP3. After $1 \mathrm{~h}$, the reaction was diluted with $\mathrm{CH}_{2} \mathrm{Cl}_{2}$ and quenched with $\mathrm{Na}_{2} \mathrm{~S}_{2} \mathrm{O}_{3}(20 \%$, aq). The aqueous layer was extracted $3 x$ with $\mathrm{CH}_{2} \mathrm{Cl}_{2}(5 \mathrm{~mL})$. The organic layer was concentrated, and the crude mixture was analyzed by ${ }^{1} \mathrm{H}$-NMR using dichloroethane as internal standard. Starting cyclohexylmethyl acetate was observed as $100 \%$ with no product formation.

Analysis: Modeled after imidate 1, the ester contains a tertiary $\mathrm{C}-\mathrm{H}$ of similar strength. If the $\mathrm{C}-\mathrm{H}$ abstraction were to occur by intermolecular HAT from an in situ generated alkoxy radical, an alkyl iodide product should be observed. However, when subjected to reaction conditions, $100 \%$ of the starting material remained, indicating this intermolecular HAT is likely not operating in the reaction. These data support directed reactivity via an imidate radical. 


\section{Probing allyl imidate as a competent intermediate}<smiles>N=C(OCC1CCCCC1)C(Cl)(Cl)Cl</smiles>

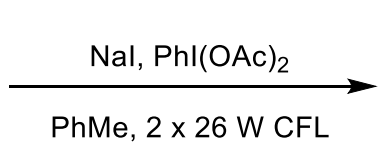<smiles>N=C(OCC1=CCCCC1)C(Cl)(Cl)Cl</smiles>

$50 \%$ iodide intermediate (4)

$12 \%$ S.M. (1)

$7 \%$ amino-iodinated product (2)

$4 \%$ olefin (5)

\section{cyclohex-1-en-1-ylmethyl 2,2,2-trichloroacetimidate (5)}

To a 2-dram vial equipped with a PTFE septum cap and magnetic stir bar, was added imidate 1 (51.7 mg, $0.2 \mathrm{mmol}$ ), iodobenzene diacetate (193 mg. $0.6 \mathrm{mmol}$ ) and $\mathrm{Nal}$ (90 $\mathrm{mg}, 0.6 \mathrm{mmol}$ ). This vial was evacuated and backfilled with $\mathrm{N}_{2}(3 \mathrm{x})$. Dry, degassed PhMe $(1 \mathrm{~mL})$ was added to the vial under $\mathrm{N}_{2}$. The reaction was irradiated with two $26 \mathrm{~W}$ compact fluorescent light bulbs and cooled by two fans for 2.66h. Upon completion, the solution was quenched via a reductive workup with $20 \% \mathrm{Na}_{2} \mathrm{~S}_{2} \mathrm{O}_{3}$. The organic layer was extracted with EtOAc (3x). The combined organic layer was concentrated in vacuo to afford $4 \%$ of the observed desaturated product 5 , along with $50 \%$ of the alkyl iodide intermediate $4,12 \%$ remaining starting material 1 , and $7 \%$ of the amino-iodinated product 2.

Note: PhMe was used as a solvent in lieu of ${ }^{\mathrm{t}} \mathrm{BuOH}$ because the rate of the reaction was observed to be slower in PhMe, allowing for observation of the desaturated product in-situ.

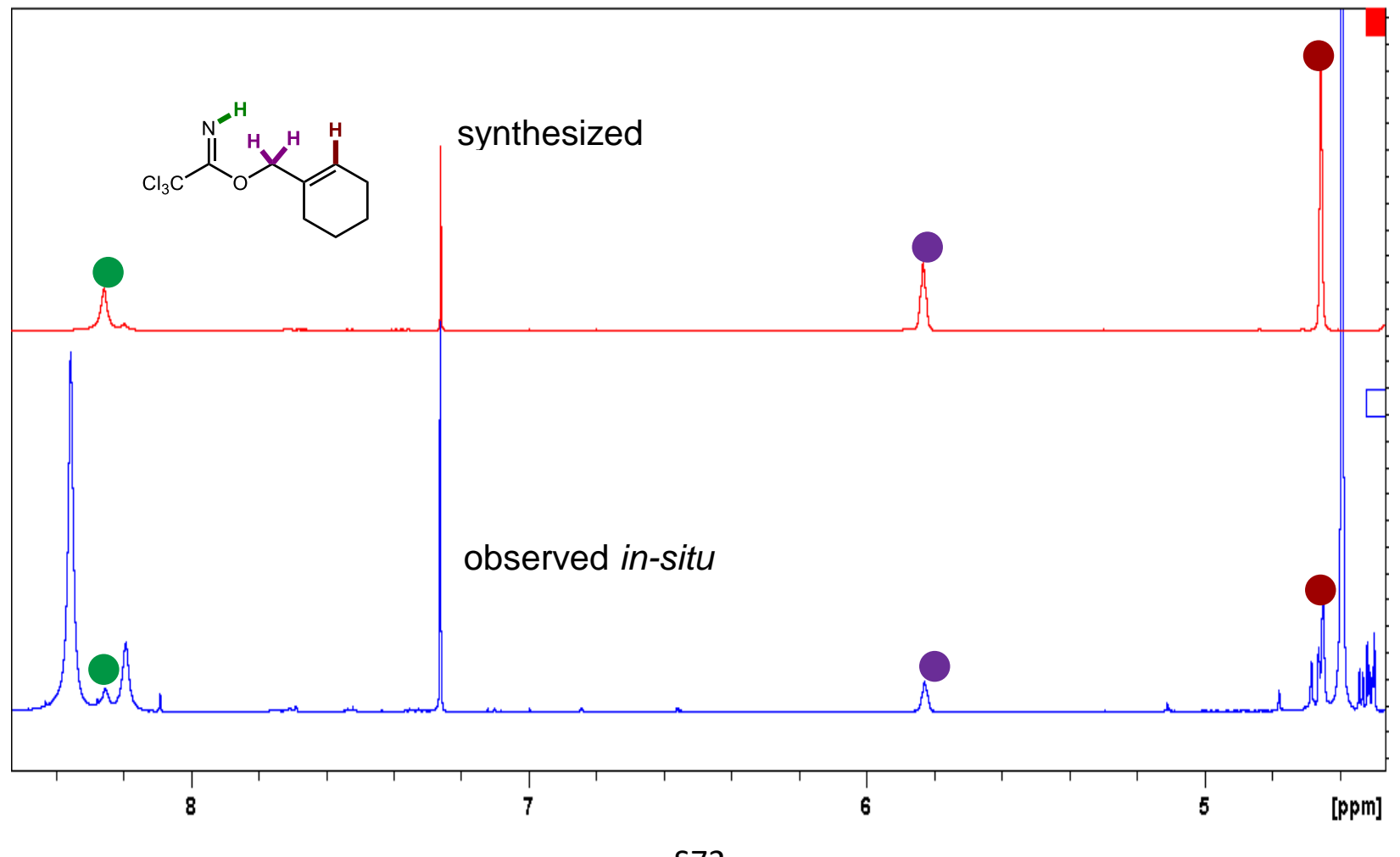




\section{Subjecting allyl imidate to modified reaction conditions}

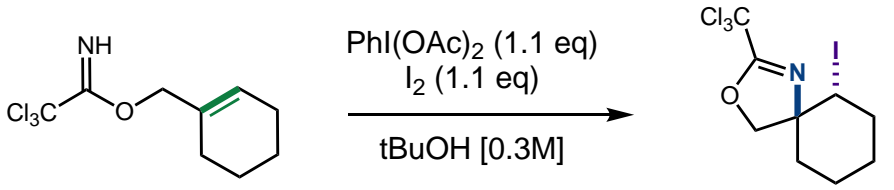

To a 2-dram vial equipped with a PTFE septum cap and magnetic stir bar was added Cyclohexenylmethanol imidate $5(51.3 \mathrm{mg}, 0.2 \mathrm{mmol})$ and ${ }^{\mathrm{B}} \mathrm{BuOH}(0.7 \mathrm{~mL})$. lodobenzene diacetate $(71 \mathrm{mg}, 0.22 \mathrm{mmol})$ and $\mathrm{I}_{2}(56 \mathrm{mg}, 0.22 \mathrm{mmol})$ were added, and the reaction was irriadiated with $2 \times 26 \mathrm{~W}$ CFLs, cooled with $2 x$ fans, and stirred at room temperature for 30 mins. Upon completion, the solution was quenched with $\mathrm{Na}_{2} \mathrm{~S}_{2} \mathrm{O}_{3}(20 \%$, aq), and the aqueous layer was extracted with $\mathrm{CH}_{2} \mathrm{Cl}_{2} 3 \times(5 \mathrm{~mL})$. The combined organic layer was concentrated in vacuo, and the crude mixture was analyzed by ${ }^{1} \mathrm{H}-\mathrm{NMR}$ using dichloroethane as an internal standard, showing 59\% (>20:1 dr) of the title compound 2.

Note 1: In a separate control experiment, it was found that $I_{2}$ rapidly decomposes the allyl imidate when iodobenzene diacetate is not present. For this reason, no prestir was used in this experiment.

Note 2: When subjecting allyl imidate 5 to standard reaction conditions (GP3), low mass balance was observed. We attribute this to the high level of oxidant present in the mixture. As evident from the reaction profile, there is a high concentration of alkyl iodide intermediate generated before any olefin intermediate is formed. Because of this, $\mathrm{I}_{2}$ in the reaction has been at least partially consumed before olefin is generated.

Analysis: allyl imidate $\mathbf{5}$ is a competent intermediate in this reaction.

On the Origin of Diastereoselectivity
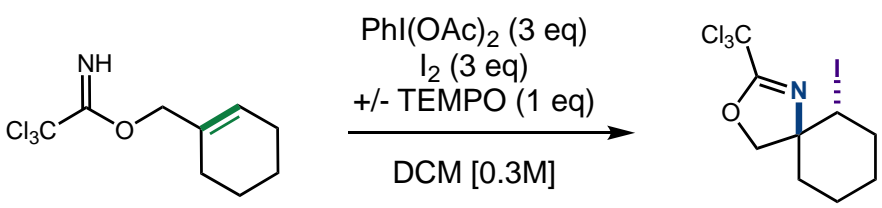

(Procedure 1) To a 2-dram vial equipped with a PTFE septum cap and magnetic stir bar was added Cyclohexenylmethanol imidate $5(51.3 \mathrm{mg}, 0.2 \mathrm{mmol})$ and $\mathrm{CH}_{2} \mathrm{Cl}_{2}(0.7 \mathrm{~mL})$. lodobenzene diacetate $(193 \mathrm{mg}, 0.6 \mathrm{mmol}), \mathrm{I}_{2}(152 \mathrm{mg}, 0.6 \mathrm{mmol})$, and TEMPO (31 mg, $0.2 \mathrm{mmol}$ ) were added. The reaction was irriadiated with $2 x 26 \mathrm{~W}$ CFLs, cooled with $2 x$ fans, and stirred at room temperature for 30 mins. Upon completion, the solution was quenched with $\mathrm{Na}_{2} \mathrm{~S}_{2} \mathrm{O}_{3}\left(20 \%\right.$, aq), and the aqueous layer was extracted with $\mathrm{CH}_{2} \mathrm{Cl}_{2} 3 x$ $(5 \mathrm{~mL})$. The combined organic layer was concentrated in vacuo, and the crude mixture was analyzed by ${ }^{1} \mathrm{H}$-NMR using dichloroethane as an internal standard, showing $84 \%$ $(>20: 1)$ of the title compound 2 . 
(Procedure 2) To a 2-dram vial equipped with a PTFE septum cap and magnetic stir bar was added Cyclohexenylmethanol imidate $5(51.3 \mathrm{mg}, 0.2 \mathrm{mmol})$ and $\mathrm{CH}_{2} \mathrm{Cl}_{2}(0.7 \mathrm{~mL})$. lodobenzene diacetate $(193 \mathrm{mg}, 0.6 \mathrm{mmol})$, and $\mathrm{I}_{2}(152 \mathrm{mg}, 0.6 \mathrm{mmol}$ ) were added to the vial. The reaction was irriadiated with $2 x 26 \mathrm{~W}$ CFLs, cooled with $2 x$ fans, and stirred at room temperature for 30 mins. Upon completion, the solution was quenched with $\mathrm{Na}_{2} \mathrm{~S}_{2} \mathrm{O}_{3}$ $\left(20 \%\right.$, aq), and the aqueous layer was extracted with $\mathrm{CH}_{2} \mathrm{Cl}_{2} 3 \times(5 \mathrm{~mL})$. The combined organic layer was concentrated in vacuo, and the crude mixture was analyzed by ${ }^{1} \mathrm{H}-\mathrm{NMR}$ using dichloroethane as an internal standard, showing $83 \%(5: 1)$ of the title compound 2.

Note: In a separate control experiment, it was found that $I_{2}$ rapidly decomposes the allyl imidate when iodobenzene diacetate is not present. For this reason, no prestir was used in this experiment.

Analysis: Using $\mathrm{CH}_{2} \mathrm{Cl}_{2}$ as a solvent in this reaction consistently yielded the title compound in $5: 1 \mathrm{dr}$. As no prestir is used in these experiments, all $\mathrm{I}_{2}$ is not fully solubilized. Because of this, a lower diastereomeric ratio is observed, likely due to competing radical and ionic mechanistic pathways. With the addition of a radical trap, we believe the diastereomeric ratio is greatly increased $(>20: 1)$ due to suppression of the radical-mediated 5-exo-trig cyclization pathway. Based on these data, we believe our amino-iodination reaction proceeds through an ionic cyclization, resulting in increased diastereomeric ratios. This is supported by enhanced solubility of $\mathrm{I}_{2}$ in ${ }^{t} \mathrm{BuOH}$ over $\mathrm{CH}_{2} \mathrm{Cl}_{2}$.

\section{Probing an "amination-first" mechanism}

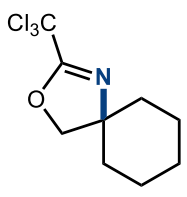

\section{2-(trichloromethyl)-3-oxa-1-azaspiro[4.5]dec-1-ene (3)}

To a 2-dram vial equipped with a PTFE septum cap and magnetic stir bar, was added imidate 3 (51.7 mg, $0.2 \mathrm{mmol}$ ), iodobenzene diacetate (193 mg. $0.6 \mathrm{mmol}$ ) and $\mathrm{Nal}(90$ $\mathrm{mg}, 0.6 \mathrm{mmol}$ ). This vial was evacuated and backfilled with $\mathrm{N}_{2}(3 \mathrm{x})$. Dry, degassed hexafluoro-2-propanol $(0.75 \mathrm{~mL})$ and $\mathrm{CH}_{2} \mathrm{Cl}_{2}(0.25 \mathrm{~mL})$ was added to the vial under $\mathrm{N}_{2}$. The reaction was irradiated with two $26 \mathrm{~W}$ compact fluorescent light bulbs and cooled by two fans for $1 \mathrm{~h}$. Upon completion, the solution was quenched via a reductive workup with $20 \% \mathrm{Na}_{2} \mathrm{~S}_{2} \mathrm{O}_{3}$. The aqueous layer was extracted with EtOAc (3x). The combined organic layer was concentrated in vacuo before purification by flash chromatography $\left(1 \% \mathrm{EtOAc} /\right.$ hexanes with $\left.1 \% \mathrm{Et}_{3} \mathrm{~N}\right)$ to afford aminated product $3(30.1 \mathrm{mg}, 59 \%)$ as a yellow oil.

Rf: 0.27 (5\% EtOAc/hexanes)

${ }^{1} \mathrm{H}$ NMR $\left(400 \mathrm{MHz}, \mathrm{CDCl}_{3}\right): \delta=4.32(\mathrm{~s}, 2 \mathrm{H}), 1.83-1.76(\mathrm{~m}, 4 \mathrm{H}), 1.66-1.54(\mathrm{~m}, 3 \mathrm{H})$, $1.40-1.23(\mathrm{~m}, 3 \mathrm{H})$. 
${ }^{13} \mathrm{C}$ NMR (100 MHz, $\left.\mathrm{CDCl}_{3}\right): \delta=160.71,87.05,80.43,72.42,36.62,25.00,22.73$.

HRMS (ESI-TOF) m/z: calc'd for $\mathrm{C}_{9} \mathrm{H}_{12} \mathrm{Cl}_{3} \mathrm{NO}[\mathrm{M}+\mathrm{H}]^{+}$256.0057, found 256.0032 .

IR (neat) cm$^{-1}:$ 2931, 2856, 1766, 1661, 1450, 1358, 1230, 994.

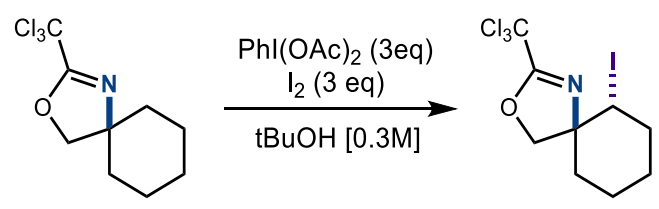

Amination product $3(25.3 \mathrm{mg}, 0.1 \mathrm{mmol})$ was subjected to GP3. Upon completion, the combined organic layer was concentrated in vacuo to achieve recovery of the starting material $3\left(77 \%-{ }^{1} \mathrm{H}-\mathrm{NMR}\right)$.

Analysis: The aminated product was subjected to reaction conditions to test the hypothesis that the amination product could undergo $\beta$ scission to generate a reactive olefin that would then be amino-iodinated in an "amination first" mechanism. No target molecule was formed indicating this side product does not lead to target product.

\section{Examining elimination mechanism}<smiles>N=C(OCC1CCCCC1)C(Cl)(Cl)Cl</smiles>

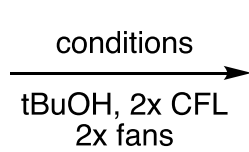<smiles>N=C(OCC1=CCCCC1)C(Cl)(Cl)Cl</smiles><smiles>ClC1=NC2(CCCC[C@H]2I)CO1</smiles>

(Procedure 1) Cyclohexenylmethanol imidate $1(51.3 \mathrm{mg}, 0.2 \mathrm{mmol})$ was subjected to GP3. Upon completion, the solution was quenched with $\mathrm{Na}_{2} \mathrm{~S}_{2} \mathrm{O}_{3}(20 \%$, aq), and the organic layer was extracted with $\mathrm{CH}_{2} \mathrm{Cl}_{2} 3 \times(5 \mathrm{~mL})$. The combined organic layer was concentrated in vacuo, and the crude mixture was analyzed by ${ }^{1} \mathrm{H}-\mathrm{NMR}$ using dichloroethane as an internal standard. The results are tabulated below.

Entry
1
2
3
4
5
6
7
8

Additive $\mathrm{NaOAc}$ $\mathrm{HOAC}$ $\mathrm{NaOAc} / \mathrm{I}_{2}$ $\mathrm{HOAc} / \mathrm{I}_{2}$ $\mathrm{NaOAc} / \mathrm{Phl}(\mathrm{OAc})_{2}$ $\mathrm{HOAc} / \mathrm{Phl}(\mathrm{OAc})_{2}$ $\mathrm{I}_{2}$ $\mathrm{Phl}(\mathrm{OAc})_{2}$
Starting Material $100 \%$ $100 \%$ $86 \%$ (13\% iodoamination) $100 \%$ $100 \%$ $100 \%$ $100 \%$ $100 \%$ 
Analysis: These experiments served as controls to probe is the starting material imidate is consumed under modified reaction conditions. This was critical, as the tertiary iodide intermediate is not isolable from starting imidate, so a mixture of the two was used in following experiments. If the starting material imidate had been consumed under modified conditions, false positive results may be observed. Entry 3 was the only set of conditions that gave conversion of starting material to any extent; a mixture of $\mathrm{NaOAc}$ and $\mathrm{I}_{2}$ is able to promote some conversion of starting material to product $\mathbf{2}$. This is likely due to small amounts of AcOl being generated throughout the course of the reaction.
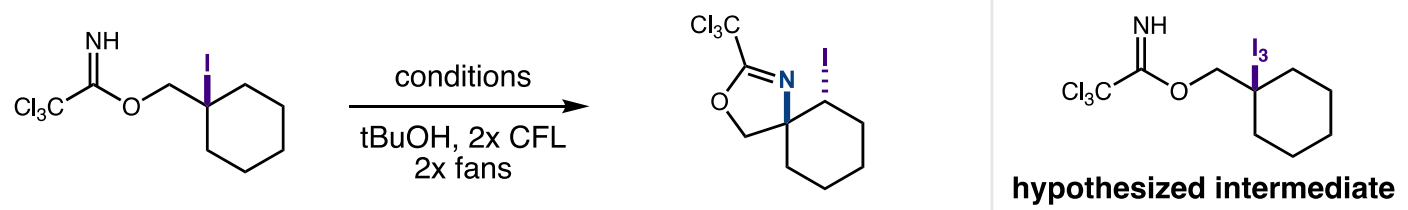

(Procedure 2) To a 2-dram vial equipped with a PTFE septum cap and magnetic stir bar was added iodinated starting material (4) $(0.2 \mathrm{mmol}, 3: 1$ mixture with imidate (1) and ${ }^{t} \mathrm{BuOH}(0.7 \mathrm{~mL})$. The desired additive(s) were added ( 3 equiv) to the vial. The reaction was irriadiated with $2 x 26 \mathrm{~W}$ CFLs, cooled with $2 x$ fans, and stirred at room temperature for $1 \mathrm{~h}$. Upon completion, the solution was quenched with $\mathrm{Na}_{2} \mathrm{~S}_{2} \mathrm{O}_{3}(20 \%$, aq), and the aqueous layer was extracted with $\mathrm{CH}_{2} \mathrm{Cl}_{2} 3 \times(5 \mathrm{~mL})$. The combined organic layer was concentrated in vacuo, and the crude mixture was analyzed by ${ }^{1} \mathrm{H}-\mathrm{NMR}$ using dichloroethane as an internal standard. The results are tabulated below.

$\begin{array}{ccc}\text { Entry } & \text { Additive } & \text { Starting Material } \\ \mathbf{1} & \mathrm{NaOAc} & 100 \% \\ \mathbf{2} & \mathrm{HOAc} & 100 \% \\ \mathbf{3} & \mathrm{NaOAc}, \mathrm{I}_{2} & 63 \%(36 \% \text { iodoamination }) \\ \mathbf{H} & \mathrm{HOAc}, \mathrm{I}_{2} & 3 \% \\ \mathbf{5} & \mathrm{NaOAc}, \mathrm{Phl}(\mathrm{OAc})_{2} & 92 \% \\ \mathbf{6} & \mathrm{HOAc}, \mathrm{Phl}(\mathrm{OAc})_{2} & 94 \% \\ \mathbf{7} & \mathrm{Phl}(\mathrm{OAc})_{2} & 84 \% \\ \mathbf{8} & \mathrm{I}_{2} & 0 \% \\ 9 & \mathrm{Phl}(\mathrm{OAc})_{2}, \mathrm{I}_{2} & 0 \%(98 \% \text { iodoamination })\end{array}$

Analysis: Controls using only base or acid showed no consumption of the alkyl iodide, indicating that direct elimination is unlikely. Additionally, using $\mathrm{Phl}(\mathrm{OAc})_{2}$ does not consume starting material. This disfavors the possibility of direct oxidation of the alkyl iodide by $\mathrm{Phl}(\mathrm{OAc})_{2}$ to promote the desired elimination. However, in the presence of $\mathrm{I}_{2}$, consumption of alkyl iodide is observed, supporting an iodine mediated elimination. We believe this intermediate to be critical for this reaction mechanism, as the formation of alkyl- $I_{3}$ complexes are far more rapid with tertiary than secondary iodides. With both $I_{2}$ and $\mathrm{NaOAc}$, we hypothesize that consumption of iodine by acetate makes this elimination a slower process. As poor yields of amino-iodinated product 2 are observed (trichloroacetamide is the major byproduct), it is clear that $\mathrm{I}_{2}$ is required for the elimination step, but $\mathrm{I}_{2}$ alone is not able to perform the desired amino-iodination. When $\mathrm{I}_{2}$ and 
$\mathrm{Phl}(\mathrm{OAc})_{2}$ are used together, nearly quantitative conversion of alkyl iodide to aminoiodination is observed.

On Product Stability: Work by Keefer et al shows that alkyl iodides form complexes with $\mathrm{I}_{2}{ }^{3}$ The stability of this complex in polar solvents is relatively unaffected by the structure of the alkyl iodide. However, in non-polar solvents, this complex stability is directly related to the structure of the alkyl iodide (tertiary > secondary). This is due to the cationic character on the carbon atom, where a highly substituted cation is more stable than a less substituted cation. As solvent polarity drops, the structure of the alkyl iodide plays a large role in stabilizing the cation, whereas in polar solvents, solvation assists stabilization.
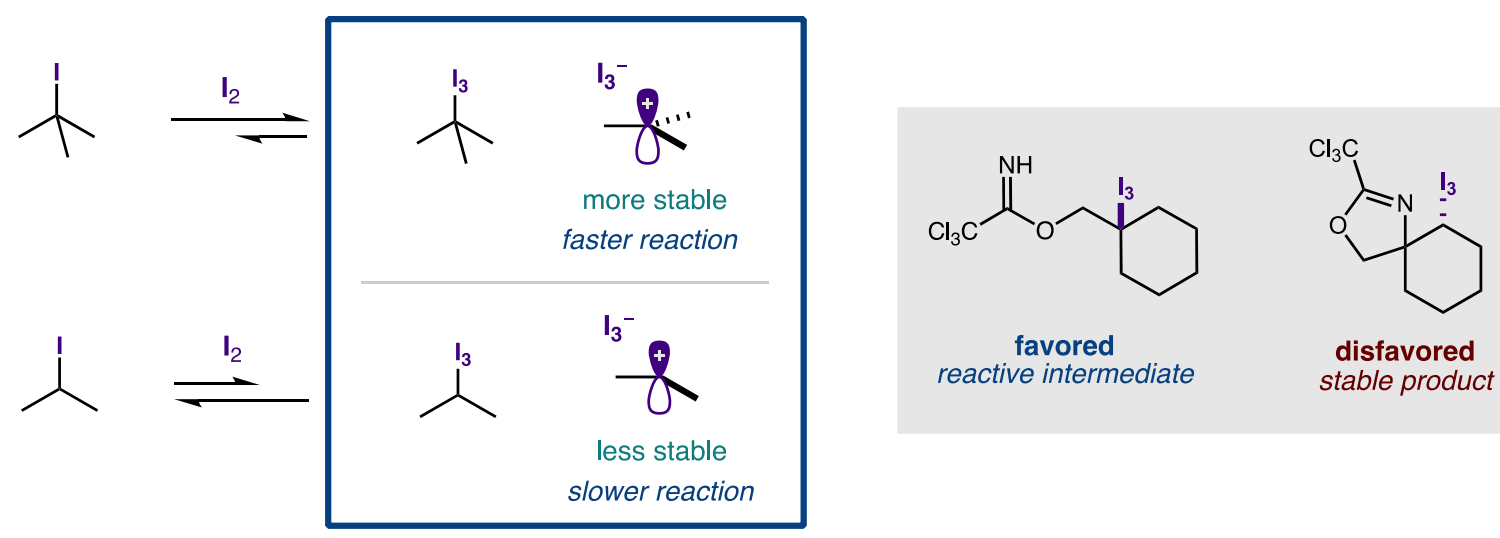

As the tertiary iodide (intermediate) forms a complex with $\mathrm{I}_{2}$ more strongly, the alkyl iodide should be expected to react faster than in the case of secondary alkyl iodides (product) leading to relative stability of products during the reaction.

${ }^{3}$ Keefer, R. M.; Andrews, L. J. J. Am. Chem. Soc. 1952, 74 (8), 1891-1893. ; Bujake, J. E.; Noyes, R. M. J. Am. Chem. Soc, 1960, 83, 1555. 


\section{UV/Vis and NMR Experiments}

UV/Vis analysis was performed on pre-stirred (5 minutes) samples of each substrate or reaction mixture dissolved in $\mathrm{CH}_{2} \mathrm{Cl}_{2}$. Each sample was prepared and diluted to the same concentrations. Below is the spectra for 2,6-lutidine with varying equivalents of $I_{2}$.

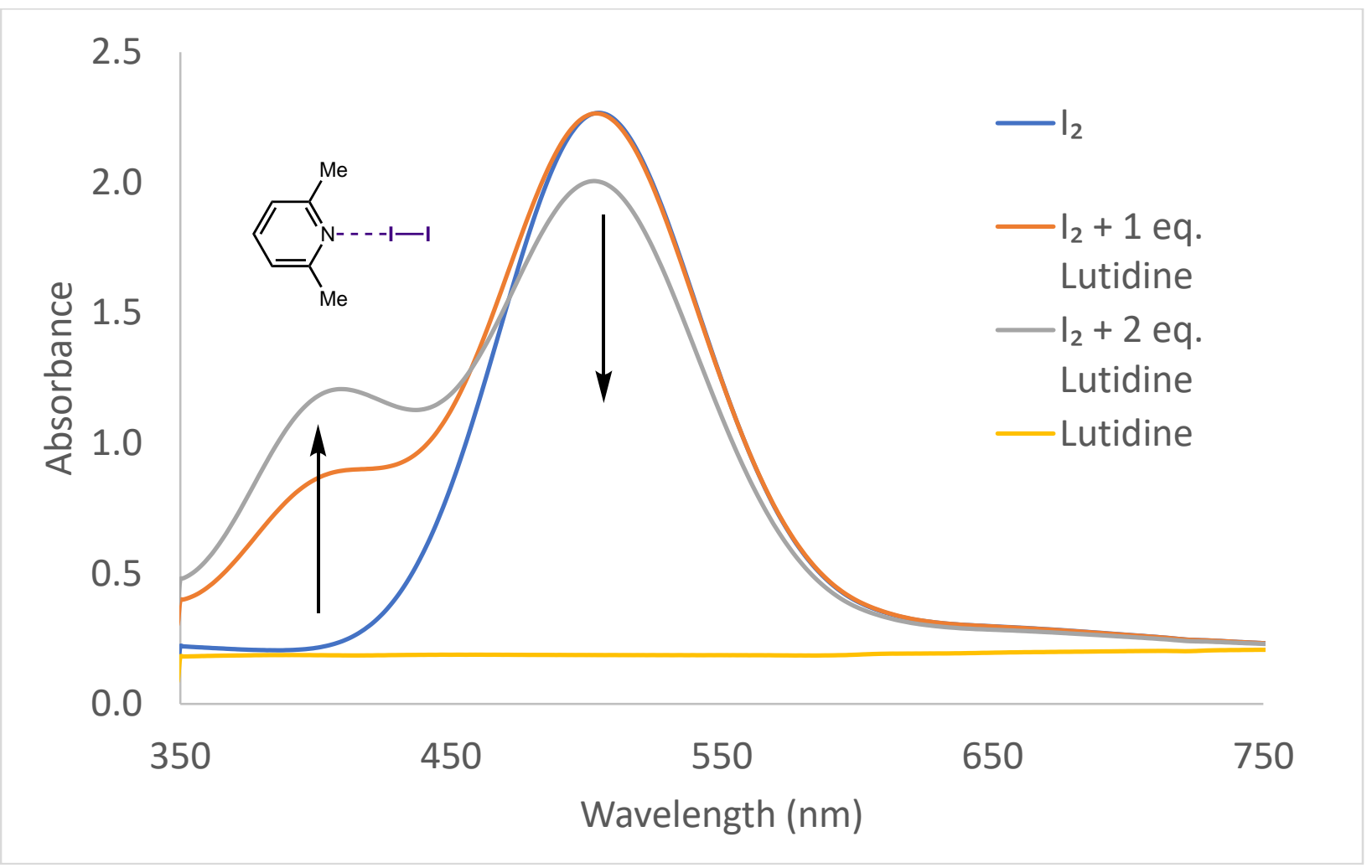

Figure 1: UV/Vis absorbance of $I_{2}$ and 2,6-lutidine.

NMR analysis was performed in pre-stirred (5 minutes) samples of substrate reaction mixture dissolved in $\mathrm{CDCl}_{3}$ and diluted to the same concentrations. Below is the spectra for 2,6-lutidine with varying equivalents of $I_{2}$. 


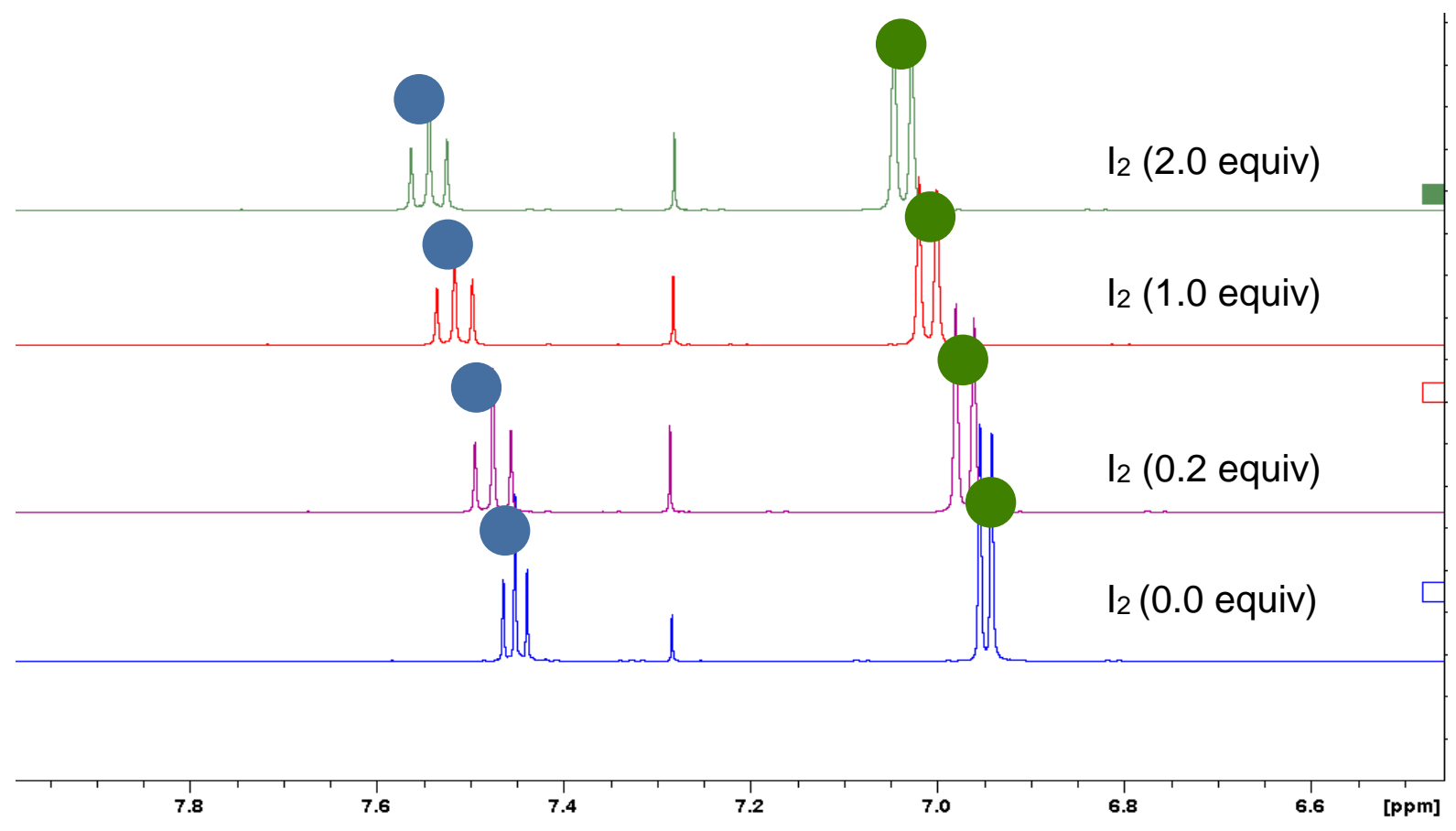

Figure 2: NMR spectra of 2,6-lutidine with varying amounts of $I_{2}$.

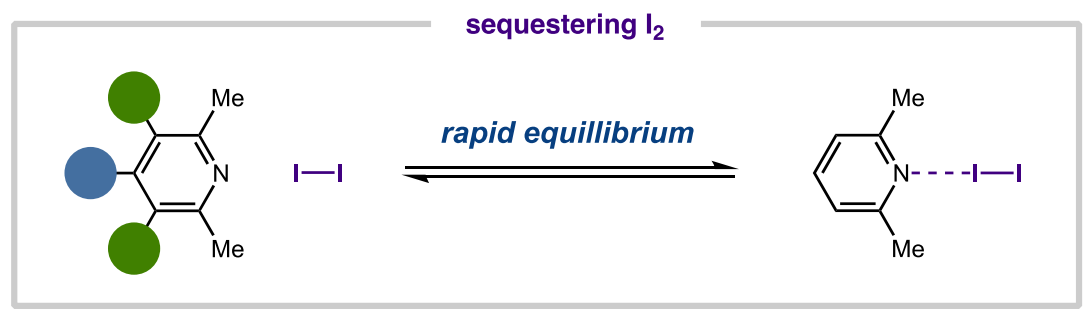

Analysis: The performed UV/Vis experiments indicate the presence of an I2-lutidine complex in solution by the appearance of a new band at $420 \mathrm{~nm} .{ }^{4}$ NMR experiments also support this complexation by the downfield shift of the aryl protons in lutidine in the presence of $\mathrm{I}_{2}$. We believe this helps explain lower diastereomeric ratios in the presence of pyridine bases by lowering the effective concentration of $\mathrm{I}_{2}$.

${ }^{4}$ Aloisi, G. G.; Beggiato, G.; Mazzucato, U. Trans. Faraday Soc. 1970, 66, 3075. 


\section{Electrochemical Data}

Cyclic voltammetry was performed with a BioLogic VSP potentiostat in a three-electrode electrochemical cell consisting of a glassy carbon disk working electrode $(0.07 \mathrm{~cm} 2$, $\mathrm{BASi}$ ), a Ag/Ag+ quasi-reference electrode (BASi) containing 0.01M AgBF4 (Sigma) in acetonitrile, and a platinum wire counter electrode $(23 \mathrm{~cm}, \mathrm{ALS})$. Charge/discharge measurements were carried out with a BioLogic VSP potentiostat in a custom glass $\mathrm{H}$ cell. Samples were prepared with $0.02 \mathrm{mmol}$ of analyte in $2.5 \mathrm{~mL}$ of $0.5 \mathrm{M}$ potassium hexafluorophosphate in dry, degassed acetonitrile. Data was analyzed using Excel. The onset potential $\left(E_{o n}\right)$ was measured by calculating the $x$-intercept potential between the linearly extrapolated fastest rising portion of the current wave (or peak) and the baseline (background) current.

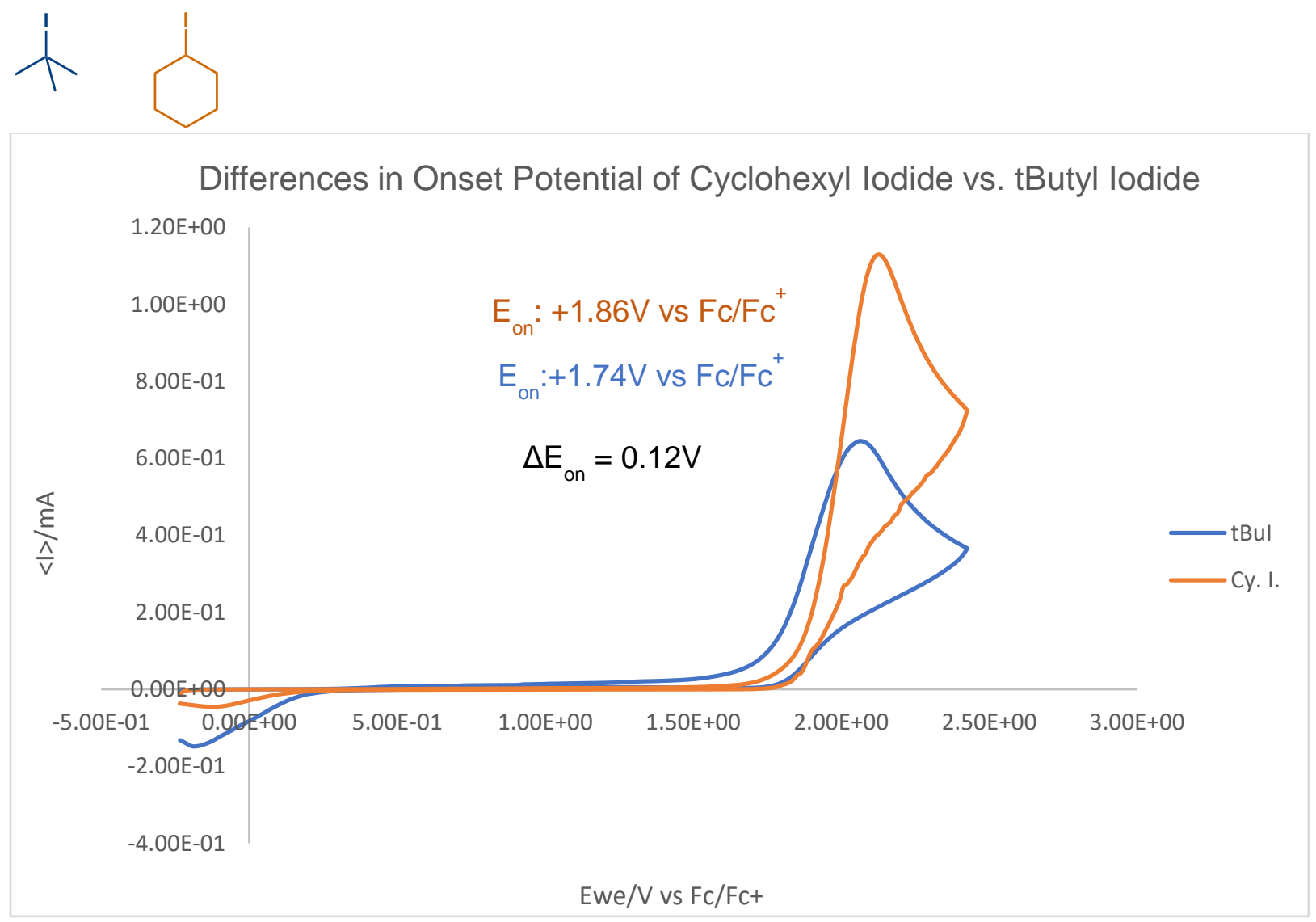

$E_{\mathrm{on}}=+1.74 \mathrm{~V}(\mathrm{tBul})$ and $+1.86 \mathrm{~V}$ (Cy. I. $)$ 


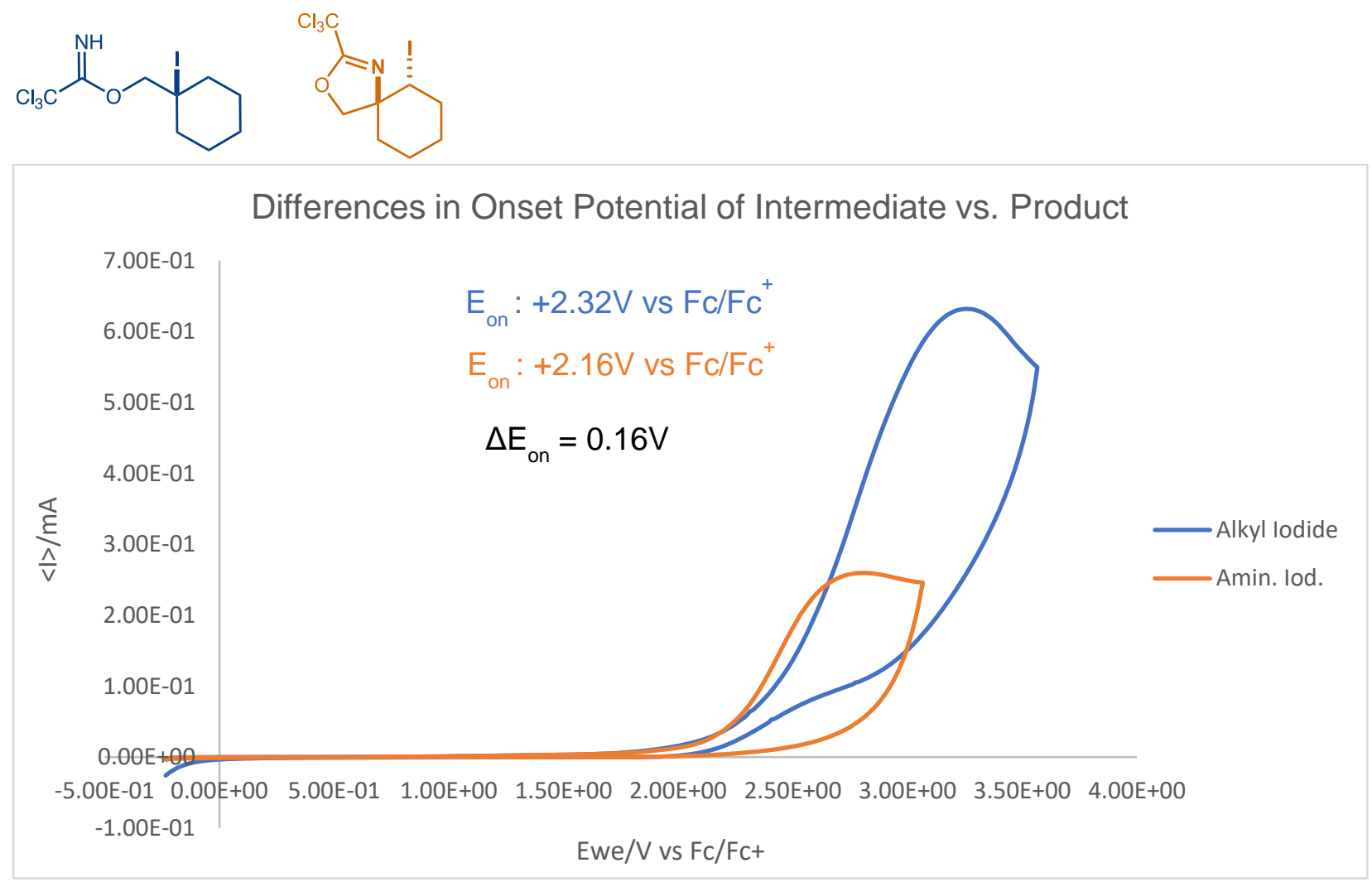

$E_{\mathrm{on}}=+2.32 \mathrm{~V}$ (alkyl iodide) and $+2.16 \mathrm{~V}$ (amino-iod.)

Analysis: Cyclic voltammetry was conducted on the alkyl iodide intermediate and the amino-iodinated product to probe if a difference in oxidation potentials was the defining factor in favoring elimination of the iodide in the intermediate and not the iodide in the product. Compared to a model cyclic voltammetry experiment between cyclobutyl iodide and tert-butyl iodide, there was no appreciable difference between the tertiary iodide generated in the intermediate and the secondary iodide generated in the amino-iodinated product. This disfavors the possibility of single electron oxidation of the alkyl iodide to promote the desired elimination. 


\section{X-ray Crystallographic Data}

A colorless block $0.21 \times 0.14 \times 0.09 \mathrm{~mm}$ in size was mounted on a Cryoloop with Paratone oil. Data were collected in a nitrogen gas stream at 100(2) K using omega scans. Crystalto-detector distance was $50 \mathrm{~mm}$ and exposure time was 0.50 seconds per frame using a scan width of $0.5^{\circ}$. Data collection was $100 \%$ complete to $26.365^{\circ}$ in $\theta$. A total of 17913 reflections were collected covering the indices $-15<=h<=15,-8<=k<=8,-16<=k<=16.2038$ reflections were founded to be symmetry independent, with an Rint of 0.0489 . Indexing and unit cell refinement indicated a primitive, monoclinic lattice. The space group was found to be P 21/n (No. 14). The data were integrated using the CrysAlis ${ }^{\text {Pro }} 1.171 .39 .46 \mathrm{e}$ software program and scaled using the SCALE3 ABSPACK scaling algorithm. Solution by intrinsic phasing (SHELXT-2015) produced a heavy-atom phasing model consistent with the proposed structure. All non-hydrogen atoms were refined anisotropically by fullmatrix least-squares (SHELXL-2014). All hydrogen atoms were placed using a riding model. Their positions were constrained relative to their parent atom using the appropriate HFIX command in SHELXL-2014. 
Table 1. Crystal data and structure refinement.

Empirical formula
Formula weight
Temperature
Wavelength
Crystal system
Space group
Unit cell dimensions

Volume

Z

Density (calculated)

Absorption coefficient

$\mathrm{F}(000)$

Crystal size

Theta range for data collection

Index ranges

Reflections collected

Independent reflections

Completeness to theta $=26.365^{\circ}$

Absorption correction

Max. and min. transmission

Refinement method

Data / restraints / parameters

Goodness-of-fit on $\mathrm{F}^{2}$

Final $R$ indices [l>2sigma(I)]

$R$ indices (all data)

Extinction coefficient

Largest diff. peak and hole
C7 H15 Cl I N O

291.55

100(2) K

$0.71073 \AA$

Monoclinic

P 21/n

$\mathrm{a}=12.0579(3) \AA$

$\alpha=90^{\circ}$.

$\mathrm{b}=6.4915(2) \AA$

$\beta=108.766(3)^{\circ}$.

$\mathrm{C}=13.4589(4) \AA$

$\gamma=90^{\circ}$.

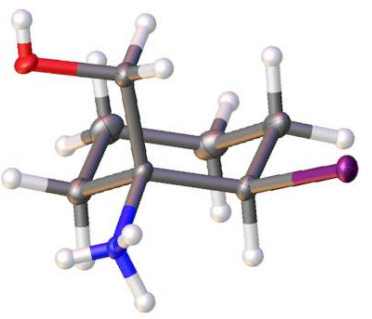

997.48(5) $\AA^{3}$

4

$1.941 \mathrm{Mg} / \mathrm{m}^{3}$

$3.429 \mathrm{~mm}^{-1}$

568

$0.210 \times 0.140 \times 0.090 \mathrm{~mm}^{3}$

3.197 to $26.365^{\circ}$.

$-15<=\mathrm{h}<=15,-8<=\mathrm{k}<=8,-16<=\mathrm{k}<=16$

17913

$2038[\mathrm{R}(\mathrm{int})=0.0489]$

$99.9 \%$

Semi-empirical from equivalents

1.00000 and 0.74075

Full-matrix least-squares on $\mathrm{F}^{2}$

2038 / 0 / 116

1.056

$\mathrm{R} 1=0.0211, \mathrm{wR} 2=0.0542$

$\mathrm{R} 1=0.0224, w R 2=0.0548$

$\mathrm{n} / \mathrm{a}$

0.995 and -0.669 e. $\AA^{-3}$ 
Table 2. Atomic coordinates $\left(\times 10^{4}\right)$ and equivalent isotropic displacement parameters $\left(\AA^{2} \times 10^{3}\right)$. $\mathrm{U}(\mathrm{eq})$ is defined as one third of the trace of the orthogonalized $\mathrm{U}^{\mathrm{ij}}$ tensor.

\begin{tabular}{lcccc}
\hline & $\mathrm{x}$ & $\mathrm{y}$ & $\mathrm{z}$ & $\mathrm{U}(\mathrm{eq})$ \\
\hline $\mathrm{C}(1)$ & $6189(2)$ & $2959(4)$ & $4479(2)$ & $16(1)$ \\
$\mathrm{C}(2)$ & $7001(2)$ & $1204(4)$ & $5022(2)$ & $13(1)$ \\
$\mathrm{C}(3)$ & $8299(2)$ & $1740(4)$ & $5274(2)$ & $15(1)$ \\
$\mathrm{C}(4)$ & $8668(2)$ & $1938(5)$ & $4294(2)$ & $20(1)$ \\
$\mathrm{C}(5)$ & $8409(3)$ & $-80(5)$ & $3666(2)$ & $23(1)$ \\
$\mathrm{C}(6)$ & $7119(3)$ & $-648(5)$ & $3390(2)$ & $22(1)$ \\
$\mathrm{C}(7)$ & $6765(2)$ & $-798(4)$ & $4372(2)$ & $16(1)$ \\
$\mathrm{Cl}(1)$ & $4113(1)$ & $5981(1)$ & $2694(1)$ & $15(1)$ \\
$\mathrm{I}(1)$ & $8801(1)$ & $4498(1)$ & $6237(1)$ & $18(1)$ \\
$\mathrm{N}(1)$ & $6769(2)$ & $732(4)$ & $6031(2)$ & $12(1)$ \\
$\mathrm{O}(1)$ & $5002(2)$ & $2328(3)$ & $4217(2)$ & $17(1)$ \\
\hline
\end{tabular}


Table 3. Bond lengths $[\AA]$ and angles $\left[^{\circ}\right]$.

\begin{tabular}{|c|c|}
\hline $\mathrm{C}(1)-\mathrm{O}(1)$ & $1.420(3)$ \\
\hline$C(1)-C(2)$ & $1.526(4)$ \\
\hline $\mathrm{C}(1)-\mathrm{H}(1 \mathrm{D})$ & 0.9900 \\
\hline$C(1)-H(1 E)$ & 0.9900 \\
\hline$C(2)-N(1)$ & $1.503(3)$ \\
\hline$C(2)-C(3)$ & $1.530(3)$ \\
\hline$C(2)-C(7)$ & $1.542(4)$ \\
\hline$C(3)-C(4)$ & $1.527(4)$ \\
\hline$C(3)-I(1)$ & $2.179(3)$ \\
\hline $\mathrm{C}(3)-\mathrm{H}(3)$ & 1.0000 \\
\hline$C(4)-C(5)$ & $1.535(4)$ \\
\hline $\mathrm{C}(4)-\mathrm{H}(4 \mathrm{~A})$ & 0.9900 \\
\hline $\mathrm{C}(4)-\mathrm{H}(4 \mathrm{~B})$ & 0.9900 \\
\hline$C(5)-C(6)$ & $1.524(4)$ \\
\hline $\mathrm{C}(5)-\mathrm{H}(5 \mathrm{~A})$ & 0.9900 \\
\hline $\mathrm{C}(5)-\mathrm{H}(5 \mathrm{~B})$ & 0.9900 \\
\hline$C(6)-C(7)$ & $1.517(4)$ \\
\hline $\mathrm{C}(6)-\mathrm{H}(6 \mathrm{~A})$ & 0.9900 \\
\hline $\mathrm{C}(6)-\mathrm{H}(6 \mathrm{~B})$ & 0.9900 \\
\hline $\mathrm{C}(7)-\mathrm{H}(7 \mathrm{~A})$ & 0.9900 \\
\hline $\mathrm{C}(7)-\mathrm{H}(7 \mathrm{~B})$ & 0.9900 \\
\hline$N(1)-H(1 A)$ & $0.87(4)$ \\
\hline$N(1)-H(1 B)$ & $0.83(4)$ \\
\hline $\mathrm{N}(1)-\mathrm{H}(1 \mathrm{C})$ & $0.73(4)$ \\
\hline $\mathrm{O}(1)-\mathrm{H}(1)$ & $0.63(3)$ \\
\hline $\mathrm{O}(1)-\mathrm{C}(1)-\mathrm{C}(2)$ & $110.1(2)$ \\
\hline $\mathrm{O}(1)-\mathrm{C}(1)-\mathrm{H}(1 \mathrm{D})$ & 109.6 \\
\hline $\mathrm{C}(2)-\mathrm{C}(1)-\mathrm{H}(1 \mathrm{D})$ & 109.6 \\
\hline $\mathrm{O}(1)-\mathrm{C}(1)-\mathrm{H}(1 \mathrm{E})$ & 109.6 \\
\hline $\mathrm{C}(2)-\mathrm{C}(1)-\mathrm{H}(1 \mathrm{E})$ & 109.6 \\
\hline$H(1 D)-C(1)-H(1 E)$ & 108.2 \\
\hline$N(1)-C(2)-C(1)$ & $107.9(2)$ \\
\hline$N(1)-C(2)-C(3)$ & $108.7(2)$ \\
\hline
\end{tabular}




\begin{tabular}{|c|c|}
\hline$C(1)-C(2)-C(3)$ & $112.9(2)$ \\
\hline$N(1)-C(2)-C(7)$ & $106.7(2)$ \\
\hline$C(1)-C(2)-C(7)$ & $112.6(2)$ \\
\hline$C(3)-C(2)-C(7)$ & $107.8(2)$ \\
\hline$C(4)-C(3)-C(2)$ & $112.9(2)$ \\
\hline$C(4)-C(3)-I(1)$ & $110.00(18)$ \\
\hline$C(2)-C(3)-I(1)$ & $112.77(17)$ \\
\hline $\mathrm{C}(4)-\mathrm{C}(3)-\mathrm{H}(3)$ & 106.9 \\
\hline $\mathrm{C}(2)-\mathrm{C}(3)-\mathrm{H}(3)$ & 106.9 \\
\hline $\mathrm{I}(1)-\mathrm{C}(3)-\mathrm{H}(3)$ & 106.9 \\
\hline$C(3)-C(4)-C(5)$ & $109.9(2)$ \\
\hline$C(3)-C(4)-H(4 A)$ & 109.7 \\
\hline $\mathrm{C}(5)-\mathrm{C}(4)-\mathrm{H}(4 \mathrm{~A})$ & 109.7 \\
\hline $\mathrm{C}(3)-\mathrm{C}(4)-\mathrm{H}(4 \mathrm{~B})$ & 109.7 \\
\hline $\mathrm{C}(5)-\mathrm{C}(4)-\mathrm{H}(4 \mathrm{~B})$ & 109.7 \\
\hline $\mathrm{H}(4 \mathrm{~A})-\mathrm{C}(4)-\mathrm{H}(4 \mathrm{~B})$ & 108.2 \\
\hline$C(6)-C(5)-C(4)$ & $110.6(2)$ \\
\hline$C(6)-C(5)-H(5 A)$ & 109.5 \\
\hline$C(4)-C(5)-H(5 A)$ & 109.5 \\
\hline $\mathrm{C}(6)-\mathrm{C}(5)-\mathrm{H}(5 \mathrm{~B})$ & 109.5 \\
\hline $\mathrm{C}(4)-\mathrm{C}(5)-\mathrm{H}(5 \mathrm{~B})$ & 109.5 \\
\hline$H(5 A)-C(5)-H(5 B)$ & 108.1 \\
\hline$C(7)-C(6)-C(5)$ & $110.8(2)$ \\
\hline $\mathrm{C}(7)-\mathrm{C}(6)-\mathrm{H}(6 \mathrm{~A})$ & 109.5 \\
\hline$C(5)-C(6)-H(6 A)$ & 109.5 \\
\hline $\mathrm{C}(7)-\mathrm{C}(6)-\mathrm{H}(6 \mathrm{~B})$ & 109.5 \\
\hline $\mathrm{C}(5)-\mathrm{C}(6)-\mathrm{H}(6 \mathrm{~B})$ & 109.5 \\
\hline$H(6 A)-C(6)-H(6 B)$ & 108.1 \\
\hline$C(6)-C(7)-C(2)$ & $112.9(2)$ \\
\hline $\mathrm{C}(6)-\mathrm{C}(7)-\mathrm{H}(7 \mathrm{~A})$ & 109.0 \\
\hline $\mathrm{C}(2)-\mathrm{C}(7)-\mathrm{H}(7 \mathrm{~A})$ & 109.0 \\
\hline $\mathrm{C}(6)-\mathrm{C}(7)-\mathrm{H}(7 \mathrm{~B})$ & 109.0 \\
\hline $\mathrm{C}(2)-\mathrm{C}(7)-\mathrm{H}(7 \mathrm{~B})$ & 109.0 \\
\hline $\mathrm{H}(7 \mathrm{~A})-\mathrm{C}(7)-\mathrm{H}(7 \mathrm{~B})$ & 107.8 \\
\hline $\mathrm{C}(2)-\mathrm{N}(1)-\mathrm{H}(1 \mathrm{~A})$ & $112(2)$ \\
\hline $\mathrm{C}(2)-\mathrm{N}(1)-\mathrm{H}(1 \mathrm{~B})$ & $115(2)$ \\
\hline
\end{tabular}




$\begin{array}{ll}H(1 A)-N(1)-H(1 B) & 104(3) \\ C(2)-N(1)-H(1 C) & 112(3) \\ H(1 A)-N(1)-H(1 C) & 104(4) \\ H(1 B)-N(1)-H(1 C) & 108(4) \\ C(1)-O(1)-H(1) & 105(3)\end{array}$

Symmetry transformations used to generate equivalent atoms:

Table 4. Anisotropic displacement parameters $\left(\AA^{2} \times 10^{3}\right)$. The anisotropic displacement factor exponent takes the form: $-2 \pi^{2}\left[h^{2} a^{* 2} U^{11}+\ldots+2 h k a^{*} b^{*} U^{12}\right]$

\begin{tabular}{lcccccc}
\hline & $\mathrm{U}^{11}$ & $\mathrm{U}^{22}$ & $\mathrm{U}^{33}$ & $\mathrm{U}^{23}$ & $\mathrm{U}^{13}$ & $\mathrm{U}^{12}$ \\
\hline $\mathrm{C}(1)$ & $12(1)$ & $16(1)$ & $18(1)$ & $3(1)$ & $1(1)$ & $-2(1)$ \\
$\mathrm{C}(2)$ & $10(1)$ & $16(1)$ & $10(1)$ & $1(1)$ & $1(1)$ & $-2(1)$ \\
$\mathrm{C}(3)$ & $12(1)$ & $17(1)$ & $14(1)$ & $0(1)$ & $1(1)$ & $-2(1)$ \\
$\mathrm{C}(4)$ & $14(1)$ & $29(2)$ & $17(1)$ & $7(1)$ & $4(1)$ & $-1(1)$ \\
$\mathrm{C}(5)$ & $21(2)$ & $32(2)$ & $16(2)$ & $3(1)$ & $7(1)$ & $5(1)$ \\
$\mathrm{C}(6)$ & $25(2)$ & $26(2)$ & $14(2)$ & $-3(1)$ & $3(1)$ & $1(1)$ \\
$\mathrm{C}(7)$ & $15(1)$ & $15(1)$ & $17(1)$ & $-1(1)$ & $2(1)$ & $-1(1)$ \\
$\mathrm{Cl}(1)$ & $15(1)$ & $13(1)$ & $13(1)$ & $-2(1)$ & $-1(1)$ & $2(1)$ \\
$\mathrm{I}(1)$ & $16(1)$ & $18(1)$ & $17(1)$ & $2(1)$ & $0(1)$ & $-6(1)$ \\
$\mathrm{N}(1)$ & $11(1)$ & $12(1)$ & $11(1)$ & $-1(1)$ & $1(1)$ & $-2(1)$ \\
$\mathrm{O}(1)$ & $10(1)$ & $16(1)$ & $20(1)$ & $7(1)$ & $-2(1)$ & $1(1)$ \\
& & & & & & \\
\hline
\end{tabular}


Table 5. Hydrogen coordinates $\left(\times 10^{4}\right)$ and isotropic displacement parameters $\left(\AA^{2} \times 10^{3}\right)$.

\begin{tabular}{llccc}
\hline & \multicolumn{1}{c}{$\mathrm{x}$} & $\mathrm{y}$ & $\mathrm{U}(\mathrm{eq})$ \\
& & & & \\
$\mathrm{H}(1 \mathrm{D})$ & 6327 & 4170 & 4951 & 19 \\
$\mathrm{H}(1 \mathrm{E})$ & 6360 & 3365 & 3834 & 19 \\
$\mathrm{H}(3)$ & 8756 & 569 & 5694 & 18 \\
$\mathrm{H}(4 \mathrm{~A})$ & 9516 & 2249 & 4500 & 24 \\
$\mathrm{H}(4 \mathrm{~B})$ & 8235 & 3085 & 3853 & 24 \\
$\mathrm{H}(5 \mathrm{~A})$ & 8613 & 79 & 3014 & 28 \\
$\mathrm{H}(5 \mathrm{~B})$ & 8898 & -1200 & 4085 & 28 \\
$\mathrm{H}(6 \mathrm{~A})$ & 6635 & 409 & 2915 & 27 \\
$\mathrm{H}(6 \mathrm{~B})$ & 6976 & -1986 & 3017 & 27 \\
$\mathrm{H}(7 \mathrm{~A})$ & 7203 & -1941 & 4813 & 20 \\
$\mathrm{H}(7 \mathrm{~B})$ & 5920 & -1129 & 4168 & 20 \\
$\mathrm{H}(1)$ & $4710(30)$ & $3040(50)$ & $3930(30)$ & $7(9)$ \\
$\mathrm{H}(1 \mathrm{~A})$ & $7360(30)$ & $120(60)$ & $6480(30)$ & $22(9)$ \\
$\mathrm{H}(1 \mathrm{~B})$ & $6650(30)$ & $1750(60)$ & $6350(30)$ & $20(9)$ \\
$\mathrm{H}(1 \mathrm{C})$ & $6280(30)$ & $20(60)$ & $5950(30)$ & $19(9)$ \\
& & & & \\
\hline
\end{tabular}


Table 6. Torsion angles $\left[{ }^{\circ}\right]$.

\begin{tabular}{lc}
\hline$O(1)-C(1)-C(2)-N(1)$ & $-63.0(3)$ \\
$O(1)-C(1)-C(2)-C(3)$ & $176.9(2)$ \\
$O(1)-C(1)-C(2)-C(7)$ & $54.5(3)$ \\
$N(1)-C(2)-C(3)-C(4)$ & $171.5(2)$ \\
$C(1)-C(2)-C(3)-C(4)$ & $-68.8(3)$ \\
$C(7)-C(2)-C(3)-C(4)$ & $56.3(3)$ \\
$N(1)-C(2)-C(3)-I(1)$ & $-63.0(2)$ \\
$C(1)-C(2)-C(3)-I(1)$ & $56.7(3)$ \\
$C(7)-C(2)-C(3)-I(1)$ & $-178.26(17)$ \\
$C(2)-C(3)-C(4)-C(5)$ & $-57.9(3)$ \\
$I(1)-C(3)-C(4)-C(5)$ & $175.11(18)$ \\
$C(3)-C(4)-C(5)-C(6)$ & $56.4(3)$ \\
$C(4)-C(5)-C(6)-C(7)$ & $-56.3(3)$ \\
$C(5)-C(6)-C(7)-C(2)$ & $57.1(3)$ \\
$N(1)-C(2)-C(7)-C(6)$ & $-172.3(2)$ \\
$C(1)-C(2)-C(7)-C(6)$ & $69.5(3)$ \\
$C(3)-C(2)-C(7)-C(6)$ & $-55.7(3)$ \\
\hline
\end{tabular}



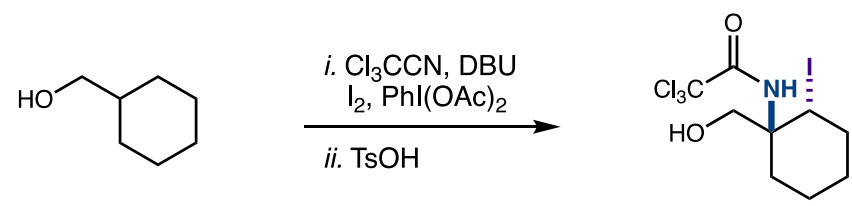

\section{2,2,2-trichloro-N-(1-(hydroxymethyl)-2-iodocyclohexyl)acetamide (55)}

Cyclohexylmethanol $(25 \mu \mathrm{L}, 0.2 \mathrm{mmol})$ was subjected to GP5. The aqueous layer was extracted $3 x$ with EtOAc $(5 \mathrm{~mL})$. The combined organic layer was concentrated in vacuo before purification by flash chromatography (isocratic: $10: 90$, EtOAc : hexanes) to afford product 55 (55.3 mg, 69\%, 3:1 diastereomeric ratio) as a pale yellow oil.

Rf: 0.37 (20\% EtOAc / hexanes).

${ }^{1} \mathbf{H}$ NMR (400 MHz, $\mathbf{C D C l}_{3}$ ): $\delta=$ Diastereomer A: $\delta=7.25$ (br s, $1 \mathrm{H}$ ), 5.09 (dd, J = 10.7 $\mathrm{Hz}, 4.3 \mathrm{~Hz}), 4.09(\mathrm{~d}, \mathrm{~J}=11.9 \mathrm{~Hz}, 1 \mathrm{H}), 3.94(\mathrm{~d}, \mathrm{~J}=11.9,1 \mathrm{H}), 2.69(\mathrm{br} \mathrm{s}, 1 \mathrm{H}), 2.38-2.32$ $(\mathrm{m}, 1 \mathrm{H}), 2.22-2.08(\mathrm{~m}, 3 \mathrm{H}), 1.74-1.49(\mathrm{~m}, 4 \mathrm{H})$.

Diastereomer B: $\delta=6.93(\mathrm{br} \mathrm{s}, 1 \mathrm{H}), 4.76(\mathrm{dd}, \mathrm{J}=11.1 \mathrm{~Hz}, 4.1 \mathrm{~Hz}, 1 \mathrm{H}), 4.13-4.07(\mathrm{~m}, 1 \mathrm{H})$, $3.64(\mathrm{dd}, \mathrm{J}=11.4 \mathrm{~Hz}, 5.9 \mathrm{~Hz}, 1 \mathrm{H}), 2.72-2.65(\mathrm{~m}, 2 \mathrm{H}), 2.38-2.32(\mathrm{~m}, 1 \mathrm{H}), 2.22-2.08(\mathrm{~m}$, $1 \mathrm{H}), 1.79-1.46(\mathrm{~m}, 4 \mathrm{H})$.

${ }^{13} \mathrm{C}$ NMR (100 MHz, $\left.\mathrm{CDCl}_{3}\right): \delta$ = Diastereomer A: $\delta=161.40,93.04,66.10,60.69,35.94$, 34.61, 30.55, 26.83, 21.55.

Diastereomer B: $\delta=161.80,93.04,66.14,61.25,39.96,36.44,28.68,27.52,21.06$.

HRMS (ESI-TOF) m/z: calc'd for $\mathrm{C}_{9} \mathrm{H}_{13} \mathrm{Cl}_{3} \mid \mathrm{NO}_{2}[\mathrm{M}+\mathrm{Na}]^{+} 421.8954$, found 421.8956 .

IR (film) cm$^{-1}:$ 3368, 2939, 2865, 1697, 1510, 818.
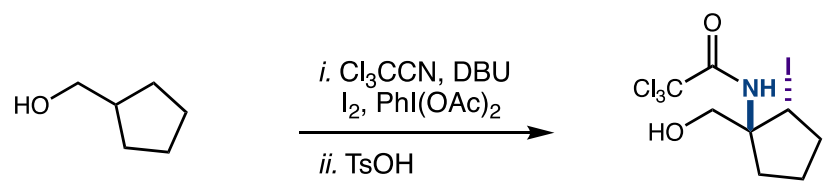

\section{2,2,2-trichloro- $N$-1-(hydroxymethyl)-2-iodocyclopentyl)acetamide (57)}

Cyclopentylmethanol ( $22 \mu \mathrm{L}, 0.2 \mathrm{mmol})$ was subjected to GP5. The aqueous layer was extracted $3 x$ with EtOAc $(5 \mathrm{~mL})$. The combined organic layer was concentrated in vacuo before purification by flash chromatography (isocratic: $10: 90$, EtOAc : hexanes) to afford product 57 (39.4 mg, 51\%, 20:1 diastereomeric ratio) as a pale yellow oil.

Rf: 0.39 (20\% EtOAc / hexanes). 
${ }^{1} \mathrm{H}$ NMR (400 MHz, $\left.\mathrm{CDCl}_{3}\right): \delta=7.43(\mathrm{br} \mathrm{s}, 1 \mathrm{H}), 4.72(\mathrm{dd}, \mathrm{J}=10.1 \mathrm{~Hz}, 7.2 \mathrm{~Hz}, 1 \mathrm{H}), 4.00$ (d, $12.0 \mathrm{~Hz}, 1 \mathrm{H}), 3.63(\mathrm{~d}, 12.0 \mathrm{~Hz}, 1 \mathrm{H}), 2.63(\mathrm{br} \mathrm{s}, 1 \mathrm{H}), 2.50-2.42(\mathrm{~m}, 1 \mathrm{H}), 2.34-2.28(\mathrm{~m}$, $1 \mathrm{H}), 2.15-1.95(\mathrm{~m}, 2 \mathrm{H}), 1.85-1.72(\mathrm{~m}, 2 \mathrm{H})$.

${ }^{13} \mathrm{C}$ NMR (100 MHz, $\left.\mathrm{CDCl}_{3}\right): \delta=161.52,92.89,69.02,66.00,37.66,31.24,30.38,23.43$.

HRMS (ESI-TOF) m/z: calc'd for $\mathrm{C}_{8} \mathrm{H}_{11} \mathrm{Cl}_{3} / \mathrm{NO}_{2}[\mathrm{M}+\mathrm{Na}]^{+}=407.8798$, found 407.8800 .

IR (film) cm$^{-1}:$ 3357, 2959, 2882, 1682, 818.
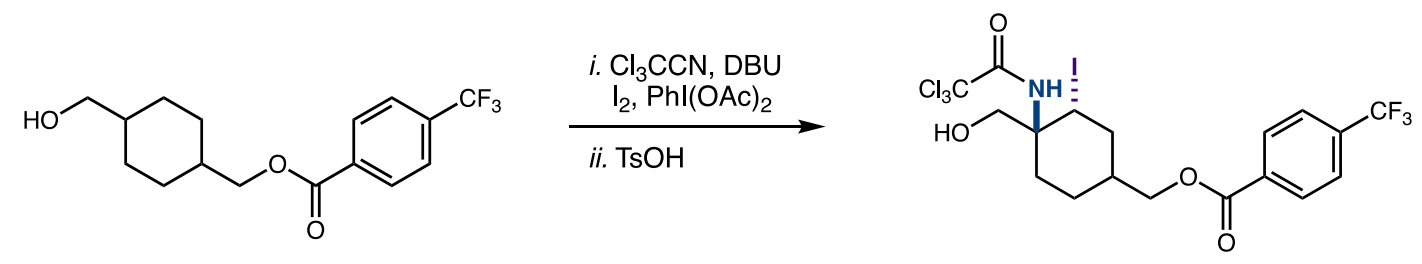

4-(hydroxymethyl)-3-iodo-4-(2,2,2-trichloroacetamido)cyclohexyl)methyl 4(trifluoromethyl)benzoate (58)

Alcohol S12A (63.3 $\mathrm{mg}, 0.2 \mathrm{mmol}$ ) was subjected to GP5. The aqueous layer was extracted $3 x$ with EtOAc $(5 \mathrm{~mL})$. The combined organic layer was concentrated in vacuo before purification by flash chromatography (isocratic: $10: 90$, EtOAc : hexanes) to afford product 58 (78.3 $\mathrm{mg}, 65 \%$, 13:1 diastereomeric ratio) as a pale yellow oil.

Rf: 0.31 (20\% EtOAc / hexanes).

${ }^{1} \mathrm{H}$ NMR $\left(400 \mathrm{MHz}, \mathrm{CDCl}_{3}\right): \delta=\delta=8.13(\mathrm{~d}, \mathrm{~J}=8.1 \mathrm{~Hz}, 2 \mathrm{H}), 8.17(\mathrm{~d}, \mathrm{~J}=8.2 \mathrm{~Hz}, 2 \mathrm{H})$, $6.71(\mathrm{br} \mathrm{s}, 1 \mathrm{H}), 5.15-5.13(\mathrm{~m}, 1 \mathrm{H}), 4.33(\mathrm{~d}, \mathrm{~J}=5.9 \mathrm{~Hz}, 2 \mathrm{H}), 4.00(\mathrm{dd}, \mathrm{J}=23.7 \mathrm{~Hz}, 11.7$ $\mathrm{Hz}, 2 \mathrm{H}), 2.54-2.42(\mathrm{~m}, 2 \mathrm{H}), 2.23-2.17(\mathrm{~m}, 2 \mathrm{H}), 2.12-1.86(\mathrm{~m}, 3 \mathrm{H}), 1.58(\mathrm{br} \mathrm{s}, 1 \mathrm{H}), 1.49-$ $1.39(\mathrm{~m}, 1 \mathrm{H})$.

${ }^{19} \mathrm{~F}$ NMR (367 MHz, CDCl3): $\delta=-63.14$.

${ }^{13} \mathrm{C}$ NMR (100 MHz, $\left.\mathrm{CDCl}_{3}\right): \delta=165.35,161.41,125.68(\mathrm{q}, \mathrm{J}=4.0 \mathrm{~Hz}), 92.75,68.36$, $60.40,35.57,33.59,29.67,27.34,23.84$.

HRMS (ESI-TOF) $\mathbf{m} / \mathbf{z}$ : calc'd for $\mathrm{C}_{18} \mathrm{H}_{18} \mathrm{Cl}_{3} \mathrm{~F}_{3} \mathrm{INO}_{4}[\mathrm{M}+\mathrm{Na}]^{+}=623.9196$, found 623.9181 .

IR (film) cm$^{-1}:$ 3310, 3014, 2989, 1723, 1683, 1080, 1013, 837. 

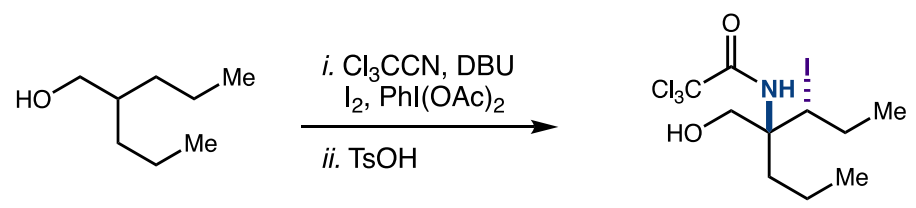

\section{2,2,2-trichloro-N-4-(hydroxymethyl)-3-iodoheptan-4-ylacetamide (59)}

2-propylpentan-1-ol (26 mg, $0.2 \mathrm{mmol}$ ) was subjected to GP5. The aqueous layer was extracted 3x with EtOAc $(5 \mathrm{~mL})$. The combined organic layer was concentrated in vacuo before purification by flash chromatography (isocratic: $10: 90$, EtOAc : hexanes) to afford product 59 (58.3 $\mathrm{mg}, 70 \%, 2: 1$ diastereomeric ratio) as a pale yellow oil.

Rf: 0.44 (20\% EtOAc / hexanes).

${ }^{1} \mathrm{H}$ NMR (400 MHz, $\mathrm{CDCl}_{3}$ ): $\delta$ = Diastereomer A: $\delta=7.34$ (br s, $\left.1 \mathrm{H}\right), 4.54$ (dd, J = 8.3 Hz, $5.5 \mathrm{~Hz}, 1 \mathrm{H}), 4.15(\mathrm{dd}, \mathrm{J}=12.2 \mathrm{~Hz}, 4.6 \mathrm{~Hz}, 1 \mathrm{H}), 3.35$ (dd, J = 9.0 Hz, 4.8 Hz), 2.18-2.10 $(\mathrm{m}, 1 \mathrm{H}), 2.00-1.31(\mathrm{~m}, 5 \mathrm{H}), 1.11(\mathrm{t}, \mathrm{J}=7.1 \mathrm{~Hz}, 3 \mathrm{H}), 0.96(\mathrm{t}, \mathrm{J}=7.2 \mathrm{~Hz}, 3 \mathrm{H})$.

Diastereomer B: $\delta=7.03(\mathrm{br} \mathrm{s}, 1 \mathrm{H}), 4.76(\mathrm{dd}, \mathrm{J}=11.3 \mathrm{~Hz}, 2.1 \mathrm{~Hz}, 1 \mathrm{H}), 4.04(\mathrm{dd}, \mathrm{J}=12.5$ $\mathrm{Hz}, 4.2 \mathrm{~Hz}, 1 \mathrm{H}), 3.94(\mathrm{dd}, \mathrm{J}=12.4 \mathrm{~Hz}, 9.8 \mathrm{~Hz}, 1 \mathrm{H}), 2.00-1.31(\mathrm{~m}, 6 \mathrm{H}), 1.11(\mathrm{t}, \mathrm{J}=7.1 \mathrm{~Hz}$, $3 \mathrm{H}), 0.99$ (t, J = 7.3 Hz, 3H).

${ }^{13} \mathrm{C}$ NMR (100 MHz, $\left.\mathrm{CDCl}_{3}\right): \delta=162.24,161.91,93.03,64.98,64.81,64.94,63.96,50.40$, 48.58, 38.74, 37.68, 28.51, 27.97, 17.03, 16.40, 15.87, 15.60, 14.64, 14.48.

HRMS (ESI-TOF) m/z: calc'd for $\mathrm{C}_{10} \mathrm{H}_{17} \mathrm{Cl}_{3} \mathrm{INO}_{2}[\mathrm{M}+\mathrm{Na}]^{+}=437.9267$, found 437.9293.

IR (film) cm-1: 3368, 2956, 2942, 2897, 1694, 868. 